\title{
Universal scalar leptoquark action for matching
}

\author{
Athanasios Dedes and Kostas Mantzaropoulos \\ Division of Theoretical Physics, Department of Physics, \\ University of Ioannina, GR 45110, Greece \\ E-mail: adedes@uoi.gr, k.mantzaropoulos@uoi.gr
}

ABSTRACT: In this study we present a universal effective action for one-loop matching of all scalar leptoquarks. We use both the Universal One-Loop Effective Action (UOLEA) and covariant diagrams to evaluate the Wilson coefficients directly in the Green basis for up to dimension- 6 operators. On the technical side, we use the newly developed method of evaluating supertraces, to further validate the results stemming from the use of covariant diagrams. As an application, we perform a fully functional matching onto Standard Model Effective Field Theory (SMEFT) of a model with two scalar leptoquark fields: a weak isospin singlet and a doublet. We demonstrate its use by calculating several observables, such as lepton magnetic and electric dipole moments, neutrino masses, proton decay rate, while we comment upon fine tuning issues in this model. Apart from its phenomenological interest, this model generates the majority of dimension- 6 operators and provides an EFT benchmark towards future matching automation.

Keywords: Beyond Standard Model, Effective Field Theories

ARXIV EPRINT: 2108.10055 


\section{Contents}

1 Introduction 1

2 Universal one loop functional matching for scalar leptoquarks 3

2.1 Tree level EFT, $\mathcal{L}_{\mathrm{EFT}}^{\text {(tree) }} 4$

$2.2 \mathrm{~K}$ - and X-matrices 5

2.3 Enumerating: UOLEA and supertraces 7

2.4 Evaluating $\mathcal{L}_{\mathrm{EFT}}^{(1-\text { loop })} \quad 9$

$\begin{array}{lll}2.5 & \text { Summary } & 13\end{array}$

3 Application: the leptoquark model $S_{1}+\tilde{S}_{2} \quad 13$

$\begin{array}{ll}3.1 \text { Lagrangian and symmetries } & 14\end{array}$

$\begin{array}{ll}3.2 & \text { Tree level matching } \\ 3.3 & \text { One }\end{array}$

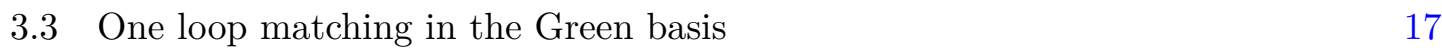

$\begin{array}{lll}3.3 .1 & \text { Renormalizable operators } & 19\end{array}$

$\begin{array}{lll}3.3 .2 & \text { Dimension-5 operator } & 19\end{array}$

$\begin{array}{lll}3.3 .3 & \text { Vector bosons-scalar operators } & 20\end{array}$

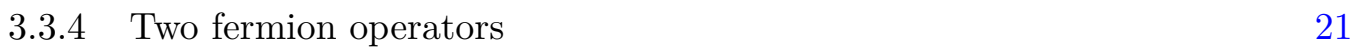

$\begin{array}{lll}3.3 .5 & \text { Four fermion operators } & 25\end{array}$

3.4 Theoretical remarks 31

3.4.1 Evanescent operators 31

3.4.2 RGE checks 32

3.5 Phenomenological aspects 33

3.5.1 Lepton magnetic and electric dipole moments 33

$\begin{array}{lll}3.5 .2 & \text { Radiative neutrino masses } & 35\end{array}$

$\begin{array}{lll}3.5 .3 & \text { Proton decay } & 36\end{array}$

$\begin{array}{lll}\text { 3.5.4 Perturbativity and fine tuning } & 37\end{array}$

4 Conclusions $\quad 39$

$\begin{array}{ll}\text { A Lagrangian and X-matrices for scalar leptoquarks } & 40\end{array}$

$\begin{array}{lll}\text { A.1 } & \mathbf{X}_{S S} & 41\end{array}$

$\begin{array}{lll}\text { A.2 } & \mathbf{X}_{S L} & 44\end{array}$

$\begin{array}{lll}\text { A.3 } & \mathbf{X}_{L S} & 46\end{array}$

$\begin{array}{llll}\text { A.4 } & \mathbf{X}_{L L} & 48\end{array}$

B Supertraces vs. covariant diagrams

C Green basis operators $\quad 55$

D Auxiliary expressions from the UOLEA 58 


\section{Introduction}

Effective field theory (EFT) [1-3] is an important part of our understanding of nature, it constitutes a robust way of dealing with new physics phenomena for Beyond the Standard Model (BSM) physics. One can obtain a low energy EFT action by integrating out heavy degrees of freedom from a, more general than the SM, UV-theory. At the end of the process we obtain an EFT Lagrangian with modified SM couplings and masses augmented by higher dimensional operators whose associated Wilson coefficients (Wcs) encode the information about the UV-theory [4]. The main technique to perform this kind of calculation has been Feynman diagrams. However, during the last decade, functional matching has seen a renewed interest.

The first steps were taken with the application of the covariant derivative expansion $(\mathrm{CDE})$ in [5-7], while the revival of these techniques and methods was recently made in $[8,9]$. A first universal result named UOLEA (Universal One Loop Effective Action) was developed in [10-12]. However, this result is not truly universal since it does not account for mixed statistics and open covariant derivatives, it can be used to decouple scalar particles only i.e. involving both heavy-heavy loops as well as heavy-light loops with the scalar particles running inside. Very recently the fermionic UOLEA was also constructed [13, 14] and the completion of the fermionic and scalar UOLEA was also developed [15] taking also mixed statistics into account.

Another approach to functional matching, which was used to derive the heavy-light part of the UOLEA, are the covariant diagrams [16], which mimic the usual Feynman diagrams but are at all steps gauge-covariant. This relatively new tool makes use of the expansion by regions [17, 18] and a simpler matching framework [19], which builds upon $[20,21]$, to further simplify the matching procedure. An example application can be found in $[16,22]$. The logic of these diagrams was taken a step forward with the development of supertrace functional-technique [23] which establishes a cleaner way to make up diagrammatic traces. Soon after that, an automated application of the CDE followed [24, 25] easing further matching calculations. Although aiming at a different direction, similar effective actions based on supertrace and Grassmannian techniques were derived in ref. [26] using a field-space super-manifold.

Briefly, the idea behind functional matching is to equate the generating functionals,

$$
\Gamma_{\mathrm{EFT}}[\phi]=\Gamma_{\mathrm{L}, \mathrm{UV}}[\phi],
$$

for the EFT and UV-theory with light fields $(\phi)$ respectively. If $S$ is a heavy field, say a leptoquark field, with mass $M_{S} \gg m_{\phi}$, then the matching conditions at tree and one-loop level read:

$$
\begin{aligned}
\mathcal{L}_{\mathrm{EFT}}^{(\text {tree })}[\phi] & =\left.\mathcal{L}_{\mathrm{UV}}[S, \phi]\right|_{S=S_{c}[\phi]}, \\
\int d^{d} x \mathcal{L}_{\mathrm{EFT}}^{(1-\text { loop })}[\phi] & =\left.\Gamma_{\mathrm{L}, \mathrm{UV}}[\phi]\right|_{\text {hard }} .
\end{aligned}
$$

Here $S_{c}[\phi]$ is the classical heavy field which solves the classical equations of motion (EOMs),

$$
\left.\frac{\delta \mathcal{S}_{\mathrm{UV}}[S, \phi]}{\delta S}\right|_{S=S_{c}[\phi]}=0
$$


Moreover, the evaluation of the loop-integral in the r.h.s. of eq. (1.3) is performed in the (hard) region assuming momenta $q \sim M_{S} \gg m_{\phi}$ and has the form

$$
\int d^{d} x \mathcal{L}_{\text {EFT }}^{(1-\text { loop })}[\phi]=\left.\frac{i}{2} \mathrm{~S} \operatorname{Tr} \log \mathbf{K}\right|_{\text {hard }}-\left.\frac{i}{2} \sum_{n=1}^{\infty} \frac{1}{n} \mathrm{~S} \operatorname{Tr}\left[\left(\mathbf{K}^{-1} \mathbf{X}\right)^{n}\right]\right|_{\text {hard }} .
$$

Therefore, the EFT Lagrangian is a sum of functional Supertraces through the log-function of the propagator $\mathbf{K}$ in field space and a power expansion of the operator $\left(\mathbf{K}^{-1} \mathbf{X}\right)$, where $\mathrm{X}$ is a field operator - an interaction matrix - evaluated at $S=S_{c}[\phi]$. Basically, finding the $\mathbf{X}$-matrix, and evaluating the Supertrace functional at the desired order in the EFT Lagrangian is what is required for the master formula of eq. (1.5) to work. This is the functional approach mainly of refs. $[16,23]$ that we use in our work here in order to

1. derive a universal one-loop effective action up-to dimension-6 operators for all scalar leptoquark (LQ) extensions of the Standard Model (SM) [27].

2. apply the formalism in the decoupling of two heavy LQ fields, a coloured weak isospin singlet $\left(S_{1}\right)$ and a coloured weak isospin doublet $\left(\tilde{S}_{2}\right)$ and derive the full set of $d \leq 6$ operators, not resorting to Baryon or Lepton number conservation.

3. support the usefulness and clarity of functional matching over traditional Feynman diagrammatic methods or within functional methods, by comparing both supertrace and covariant diagrammatic techniques.

There are, various worked out examples functionally integrating out non-degenerate fields in refs. $[10,13,15]$, however, with an exception of ref. [22] and to our knowledge, there is no other functional calculation with two-field decoupling and more general Yukawa interactions in the literature as the one we present here. ${ }^{1}$ The renormalization scheme in our calculation is a (modified) mass independent one $(\overline{\mathrm{MS}})$ and we regulate the integrals with dimensional regularization. We match on to SMEFT operators within a redundant basis, referred to as Green (or General) basis, which consists of operators written before equations of motion for the light-fields are taken into account $[32,33]$. Expressions for translating Wcs from Green to Warsaw basis [34] are given in ref. [33].

However, before taking up the above analysis, we first validated calculations performed with Feynman diagrammatic techniques. We started from matching a single charged singlet, the model of ref. [35] in SMEFT. We found full agreement apart from a missing operator [36]. Next, we applied functional covariant diagrams to a benchmark leptoquark model $S_{1}+S_{3}$ of ref. [33], where we found perfect agreement with ref. [33]. Part of our functional calculation in our paper here addresses this model too but now with the inclusion of Baryon number violating terms in the UV-Lagrangian. Finally, regarding the tree-level part of our calculation we found agreement with ref. [37].

\footnotetext{
${ }^{1}$ A complete one-loop functional matching of the singlet scalar extension of the SM exists in ref. [23] and very recently, there have also been two complete one loop, but one-field-type, matching calculations using functional methods, where ref. [28] matches the Type-I neutrino seesaw onto SMEFT, while ref. [29] matches the Higgs triplet extension of the electroweak gauge sector. Also recently, one-field heavy scalar decoupling has been classified in ref. [30] by using the code of ref. [31].
} 


\begin{tabular}{|c|ccc|}
\hline LQ-fields $(S)$ & $\mathrm{SU}(3)$ & $\mathrm{SU}(2)$ & $\mathrm{U}(1)$ \\
\hline$S_{1}$ & $\overline{3}$ & 1 & $\frac{1}{3}$ \\
$\tilde{S}_{1}$ & $\overline{3}$ & 1 & $\frac{4}{3}$ \\
$S_{2}$ & 3 & 2 & $\frac{7}{6}$ \\
$\tilde{S}_{2}$ & 3 & 2 & $\frac{1}{6}$ \\
$S_{3}$ & $\overline{3}$ & 3 & $\frac{1}{3}$ \\
\hline
\end{tabular}

Table 1. All possible representations of leptoquark fields under the SM gauge group.

We have chosen to study the decoupling of heavy scalar leptoquark fields for two main reasons: first, there is a plethora of interesting BSM phenomena associated to them, i.e. from neutrino masses and proton decay [38], to possible interpretation of recent flavour anomalies and enhanced anomalous magnetic moment of the muon [39-44], and second, leptoquark fields are naturally embedded in Grand Unified Theories (GUTs) which may be in turn linked to even more fundamental theories.

\section{Universal one loop functional matching for scalar leptoquarks}

Leptoquarks $(S)$ are hypothetical fields defined by their Yukawa interactions to both SM quarks and leptons via the Lagrangian,

$$
\mathcal{L}_{\mathrm{S}-\mathrm{f}}=\bar{F}^{c} \boldsymbol{\lambda}_{i}^{L} F S_{i}+\bar{f}^{c} \boldsymbol{\lambda}_{i}^{R} f S_{i}+\bar{f} \tilde{\boldsymbol{\lambda}}_{i} F S_{i}+\text { h.c. },
$$

where fermion $F=\{q, \ell\}$ is a Left handed quark or lepton weak doublet field, while $f=\{u, d, e\}$ is a Right-handed fermion weak singlet field. $F^{c}, f^{c}$ denote charge-conjugated fermion fields. Gauge and flavour indices are all suppressed in (2.1), or otherwise encoded in the Yukawa couplings, $\lambda^{L}, \lambda^{R}$ and, $\tilde{\lambda}$. Therefore, there are five different scalar leptoquark field representations in weak isospin space: three singlets, two doublets and one triplet. Their gauge quantum numbers under the SM gauge group, are shown in table $1 .^{2}$ The LQ-flavour index $i$ in (2.1) takes the values $i=\{1, \tilde{1}, 2, \tilde{2}, 3\} .^{3}$

Obviously, by picking up only quarks from $F-$ (or from $f-$ ) fields we arrive at Baryon $(B)$ and Lepton $(L)$ number non-conservation LQ-interactions.

All five scalar LQs can interact with the SM Higgs-field ${ }^{4}(H)$ through trilinear and quadratic terms of the form:

$$
\mathcal{L}_{\mathrm{S}-\mathrm{H}}=\left(A_{i j} H^{\dagger} S_{i} S_{j}+\text { h.c. }\right)+\lambda_{H i}\left(S_{i}^{\dagger} S_{i}\right)\left(H^{\dagger} H\right)+\left(\lambda_{3 S} S_{i} S_{j} S_{k} H^{\dagger}+\text { h.c. }\right)+\ldots,
$$

where "..." mean other gauge invariant terms of the form $(S S H H)$ and $(S S S H)$. Their exact form is irrelevant for drawing the supertrace functional diagrams since their explicit details entered only at the end in $\mathbf{X}$-matrices of (1.5). Note that the $A$-term of (2.2)

\footnotetext{
${ }^{2}$ In notation of (2.1) some leptoquark fields from table 1 may be their charge-conjugated fields.

${ }^{3}$ With apologies to the reader, the LQ-flavour indices- $i, j, k$ used throughout this section should not be confused with the colour indices- $i, j, k$ introduced in section 3 .

${ }^{4}$ The hypercharge of the SM Higgs doublet is defined so that $Y_{H}=1 / 2$.
} 
has mass dimension one, there are only two options $H S_{1} \tilde{S}_{2}$ and $H S_{3} \tilde{S}_{2}$, and it plays an important role in the effective Lagrangian at $d \geq 5$ level as we shall see in the next section.

Furthermore, self-interactions among LQs read in general as

$$
\mathcal{L}_{\mathrm{S}}=-M_{i}^{2}\left|S_{i}\right|^{2}+A_{i j k}^{\prime}\left(S_{i}^{\dagger} S_{j} S_{k}\right)+c_{i j k l}\left(S_{i}^{\dagger} S_{j}\right)\left(S_{k}^{\dagger} S_{l}\right)+\cdots,
$$

where again "..." refer to different internal gauge group invariant structure of terms not in our immediate interest in constructing the effective action. Again $A^{\prime}$ is a mass dimension one parameter but break baryon and lepton numbers. Among the fields arranged in table 1, there are three choices of $A^{\prime}$-terms: $S_{1}^{\dagger} \tilde{S}_{2} \tilde{S}_{2}, S_{3}^{\dagger} \tilde{S}_{2} \tilde{S}_{2}$ and $\tilde{S}_{1}^{\dagger} S_{2} \tilde{S}_{2}$. Masses $M_{i}$ in $(2.3)$ are assumed much heavier than the electroweak scale $m_{W}$ but the dimension-full parameters introduced above could in general range within

$$
0 \leq\left\{A / M_{i}, A^{\prime} / M_{i}\right\} \lesssim 1
$$

In total, the BSM Lagrangian is

$$
\mathcal{L}_{\mathrm{BSM}}=\mathcal{L}_{\mathrm{S}-\mathrm{f}}+\mathcal{L}_{\mathrm{S}-\mathrm{H}}+\mathcal{L}_{\mathrm{S}}
$$

This "universal" way of writing down leptoquark interactions will be the stepping stone for the calculation of the effective action at tree and one-loop levels using eqs. (1.2), (1.3) and (1.5), respectively, since these will determine the dimensionality of the $\mathbf{X}$-matrices that we will introduce shortly. Otherwise, the explicit form of $\mathcal{L}_{\mathrm{BSM}}$ is given in appendix $\mathrm{A}$.

\subsection{Tree level EFT, $\mathcal{L}_{\mathrm{EFT}}^{\text {(tree) }}$}

We start out with the UV-Lagrangian $\mathcal{L}_{\mathrm{UV}}[S, \phi]=\mathcal{L}_{\mathrm{SM}}[\phi]+\mathcal{L}_{\mathrm{BSM}}[S, \phi]$ and derive the EOMs (1.4) for the heavy fields $S_{i}$ in table 1. We solve EOMs and substitute the solutions for the classical fields $S_{i, c}[\phi]$ back into $\mathcal{L}_{U V}$ in order to obtain the tree-level EFT from eq. (1.2). By expanding the classical field in inverse powers of heavy masses $M_{i}$

$$
S_{i, c}[\phi]=S_{i, c}^{(3)}+S_{i, c}^{(4)}+\ldots
$$

we find

$$
\begin{aligned}
& \left(S_{i, c}^{(3)}\right)^{\dagger}=\frac{1}{M_{i}^{2}}\left(\bar{F}^{c} \boldsymbol{\lambda}_{i}^{L} F+\bar{f}^{c} \boldsymbol{\lambda}_{i}^{R} f+\bar{f} \tilde{\boldsymbol{\lambda}}_{i} F\right) \\
& \left(S_{i, c}^{(4)}\right)^{\dagger}=\frac{1}{M_{i}^{2}} A_{i j} H^{\dagger} S_{j, c}^{(3)}
\end{aligned}
$$

The solutions $S_{i, c}^{(n)}$ contain operators with mass dimension $n$ which are suppressed by factors that scale like $M_{i}^{n-1}$. Plugging in this back to eqs. (2.1)-(2.3) we obtain the tree-level effective Lagrangian containing $d \leq 7$ operators

$$
\mathcal{L}_{\text {EFT }}^{(\text {tree })}[\phi]=M_{i}^{2}\left(S_{i, c}^{(3)}\right)^{\dagger}\left(S_{i, c}^{(3)}\right)+\left(A_{i j} H^{\dagger} S_{i, c}^{(3)} S_{j, c}^{(3)}+\text { h.c. }\right),
$$

where $S_{i, c}^{(3)}$ is the hermitian conjugate of (2.7). Therefore, the tree-level EFT contains only four-fermion dimension- 6 operators proportional to the product of couplings from the 
set $\left\{\lambda^{L}, \lambda^{R}, \tilde{\lambda}\right\}$. On the other hand, all tree-level dimension-7 operators are proportional to the dimension-full combination of parameters $A \times \lambda^{2}$. From eq. (2.2) we see that the parameters $\lambda_{H i}$ and $\lambda_{3 S}$ appear first to multiplying operators with dimensions- 8 and 10 , respectively, while from eq. (2.3), the parameters $A^{\prime}$ and $c$ are associated with dimension-9 and 12, respectively. Although our main focus in this paper is on operators with dimensions less or equal to six it is obvious that dimension- 7 operators at tree-level may become equally important in the parameter region where $A \approx M_{i}$ and one other leptoquark mass is $M_{j} \approx \sqrt{v M_{i}}$, with $v$ being the electroweak vev.

\section{$2.2 \quad \mathrm{~K}$ - and X-matrices}

The neccessary steps for one loop matching are neatly outlined in [23] and are followed closely here. In performing the matching, the method of functional supertraces will be used as introduced in [23]. After collecting and constructing the supertraces, the application of the CDE (Covariant Derivative Expansion) is carried out automatically through two recently developed packages [24, 25]. We will be using mainly the package, STrEAM of [24].

The rationale of these diagrams comes from an earlier diagrammatic approach to matching which uses the so called covariant diagrams [16]. In appendix B we make this comparison more explicit by presenting the equivalent covariant diagrams that match to the diagrammatic supertraces.

We begin by creating field multiplets, where we denote the five (heavy) leptoquark fields, listed in table 1 , as $\left\{S_{i}\right\}=\left\{S_{1}, \tilde{S}_{1}, S_{2}, \tilde{S}_{2}, S_{3}\right\}$. Additionally, to treat chiral fermions we introduce fictitious fields promoting Weyl fermions into Dirac and properly inserting projections operators to single-out the correct chirality of the fields in the end. For simplicity these projection operators are left implicit throughout the text. The field multiplets then read,

$$
\begin{aligned}
\varphi_{S} & =\left\{\varphi_{S_{i}}\right\}=\left\{\left(\begin{array}{c}
S_{i} \\
S_{i}^{*}
\end{array}\right)\right\}, \\
\varphi_{H} & =\left\{\left(\begin{array}{c}
H \\
H^{*}
\end{array}\right)\right\}, \\
\varphi_{f} & =\left\{\varphi_{\ell}, \varphi_{q}, \varphi_{u}, \varphi_{e}, \varphi_{d}\right\}=\left\{\left(\begin{array}{c}
\ell \\
\ell^{c}
\end{array}\right),\left(\begin{array}{c}
q \\
q^{c}
\end{array}\right),\left(\begin{array}{c}
u \\
u^{c}
\end{array}\right),\left(\begin{array}{c}
e \\
e^{c}
\end{array}\right),\left(\begin{array}{c}
d \\
d^{c}
\end{array}\right)\right\}, \\
\varphi_{V} & =\{B, W, G\} .
\end{aligned}
$$

We also introduce the conjugate field multiplets,

$$
\begin{aligned}
& \bar{\varphi}_{S}=\left\{\bar{\varphi}_{S_{i}}\right\}=\left\{\left(S_{i}^{\dagger} S_{i}^{T}\right)\right\} \\
& \bar{\varphi}_{H}=\left\{\left(H^{\dagger} H^{T}\right)\right\}, \\
& \bar{\varphi}_{f}=\left\{\bar{\varphi}_{\ell}, \bar{\varphi}_{q}, \bar{\varphi}_{u}, \bar{\varphi}_{e}, \bar{\varphi}_{d}\right\}=\left\{\left(\bar{\ell} \bar{\ell}^{c}\right),\left(\bar{q} \bar{q}^{c}\right),\left(\bar{u} \bar{u}^{c}\right),\left(\bar{e} \bar{e}^{c}\right),\left(\bar{d} \bar{d}^{c}\right)\right\}, \\
& \bar{\varphi}_{V}=\{B, W, G\} .
\end{aligned}
$$


These field multiplets are connected to the inverse propagator matrix- $\mathbf{K}$ and to interaction matrix-X, both needed for master formula (1.5), via the second variation of the action as

$$
+\frac{1}{2} \delta \bar{\varphi} \mathbf{K} \delta \varphi-\frac{1}{2}\left(\begin{array}{ll}
\delta \bar{\varphi}_{S} \delta \bar{\varphi}_{L}
\end{array}\right)\left[\begin{array}{ll}
X_{S S} & X_{S L} \\
X_{L S} & X_{L L}
\end{array}\right]\left(\begin{array}{c}
\delta \varphi_{S} \\
\delta \varphi_{L}
\end{array}\right) .
$$

We have gathered all light multiplets in $\varphi_{L}$ and we denote the whole field multiplet with $\varphi$ for brevity. Matrix-K is block-diagonal with $\left(P^{2}-m^{2}\right)$ for spin-0 fields, $(\not P-m)$ for spin$1 / 2$ and $-\eta^{\mu \nu}\left(P^{2}-m^{2}\right)$ for spin-1 fields in Feynman gauge. Here $P_{\mu} \equiv i D_{\mu}$ is basically the (Hermitian) covariant derivative. The $\mathbf{X}$-matrix may contain potential-only interactions and/or terms with open covariant derivatives as well. It is evaluated with $S_{i}=S_{i, c}[\phi]$. Moreover, in the most general case,

$$
\mathbf{X}=\mathbf{U}+P^{\kappa} \mathbf{Z}_{\kappa}+\overline{\mathbf{Z}}_{\kappa} P^{\kappa}+\cdots
$$

where the dots contain terms with two or more open covariant derivatives, however these higher derivative terms do not appear in any renormalizable UV-model, such as the LQmodels under consideration and can be ignored.

The $\mathbf{X}$-interaction matrix structure in (2.18) is split into heavy-heavy, $\mathbf{X}_{S S}$, lightlight, $\mathbf{X}_{L L}$ and heavy-light (light-heavy), $\mathbf{X}_{S L}\left(\mathbf{X}_{L S}\right)$ sub-blocks. In terms of the expansion matrices of (2.19) these sub-blocks are organized in the following way,

$$
\begin{aligned}
& \left(\mathbf{X}_{S S}\right)_{10 \times 10}=\left(\left(U_{S_{i} S_{j}}\right)_{10 \times 10}\right), \\
& \left(\mathbf{X}_{S L}\right)_{10 \times 15}=\left(\left(U_{S_{i} f}\right)_{10 \times 10}\left(U_{S_{i} H}\right)_{10 \times 2}\left(X_{S_{i} V}\right)_{10 \times 3}\right) \text {, } \\
& \left(\mathbf{X}_{L S}\right)_{15 \times 10}=\left(\begin{array}{c}
\left(U_{f S_{i}}\right)_{10 \times 10} \\
\left(U_{H S_{i}}\right)_{2 \times 10} \\
\left(X_{V S_{i}}\right)_{3 \times 10}
\end{array}\right) \\
& \left(\mathbf{X}_{L L}\right)_{15 \times 15}=\left(\begin{array}{lll}
\left(U_{f f}\right)_{10 \times 10} & \left(U_{f H}\right)_{10 \times 2} & \left(U_{f V}\right)_{10 \times 3} \\
\left(U_{H f}\right)_{2 \times 10} & \left(U_{H H}\right)_{2 \times 2} & \left(U_{H V}\right)_{2 \times 3} \\
\left(U_{V f}\right)_{3 \times 10} & \left(U_{V H}\right)_{3 \times 2} & \left(U_{V V}\right)_{3 \times 3}
\end{array}\right) \text {, }
\end{aligned}
$$

where with subscript we denote the respective matrix dimensionality for each generation of light fermions.

From the general interactions, eqs. (2.1), (2.2) and (2.3), of the scalar leptoquarks outlined in the previous subsection, we can now read the mass dimensions of the corresponding elements of U-matrices. By schematically performing a second variation, for example on $S(\bar{f} f)$-terms,

$$
\begin{aligned}
\delta^{2}(S \bar{f} f) & \propto(\delta S)(\delta \bar{f}) f+(\delta S) \bar{f}(\delta f)+S(\delta \bar{f})(\delta f) \\
& =(\delta \bar{f}) U_{\bar{f} S}(\delta S)+\left(\delta S^{T}\right) U_{S^{T} f}(\delta f)+(\delta \bar{f}) U_{\bar{f} f}(\delta f)
\end{aligned}
$$

we can obtain the mass dimensions of the $\mathbf{X}$-matrices. Adding the h.c. of this interaction and doing again the exact calculation for the conjugate fermion fields, namely $S \bar{f}^{c} f^{c}+$ h.c., 
we can get the mass dimension of the matrices $\left[\mathbf{U}_{f f}\right]=3,\left[\mathbf{U}_{S_{i} f}\right]=3 / 2$ and $\left[\mathbf{U}_{f S_{i}}\right]=$ $3 / 2$. Consequently, we arrive at the following mass dimensions for all involved matrices in notation of eqs. (2.20)-(2.23),

$$
\begin{aligned}
& {\left[\mathbf{X}_{S S}\right]=[(1,2,3,4,6)],} \\
& {\left[\mathbf{X}_{S L}\right]=\left[\mathbf{X}_{L S}\right]=[3 / 2(3,4,6)(3,4)],} \\
& {\left[\mathbf{X}_{L L}\right]=\left[\mathbf{X}_{L L}\right]_{\mathrm{SM}}+\left[\mathbf{X}_{L L}\right]_{\mathrm{BSM}}=\left[\begin{array}{ccc}
1 & 3 / 2 & 3 / 2 \\
3 / 2 & 2 & 2 \\
3 / 2 & 2 & 2
\end{array}\right]+\left[\begin{array}{lll}
3 & 0 & 0 \\
0 & 6 & 0 \\
0 & 0 & 6
\end{array}\right],}
\end{aligned}
$$

where in parenthesis we denote all possible mass dimensions with $d \leq 6$ (starting from the lowest) of the $\mathbf{X}$-matrices.

For all scalar leptoquark interactions we need to calculate the explicit form of the $\mathbf{X}$-matrices in eqs. (2.20)-(2.23). This is done in appendix A. As an application, we shall deploy those matrices in section 3 , for a detailed functional matching procedure in a particular model for decoupling together two heavy LQ fields, the $S_{1}$ and the $\tilde{S}_{2}$.

\subsection{Enumerating: UOLEA and supertraces}

There are two contributions in the r.h.s. of one-loop effective action [eq. (1.5)]: the log-type term, STr $\log \mathbf{K}$, and the power-type, $\mathrm{S} \operatorname{Tr}\left[\left(\mathbf{K}^{-1} \mathbf{X}\right)^{n}\right]$. However, a great deal of contributions in eq. (1.5) are encoded in 19-UOLEA-terms [cf. eq. (2.44)] for only-heavy scalars. These UOLEA terms include the full expressions of log-type terms and all power-type diagrams with only heavy scalars in the loop.

What remains to be added is all heavy-light diagrams. For those we use the technique of functional supertraces of ref. [23] and, as a cross check, the technique of covariant diagrams of ref. [16]. In fact, a detailed diagrammatic comparison of both techniques is given in appendix B.

The $\mathbf{X}$-matrices are the building blocks for the functional supertraces. In most of the cases only the $\mathbf{U}$-matrices appear in the expansion (2.19). Different combinations of these matrices are inserted into diagrams and make up operators of up-to mass dimension- 6 . In what follows we list all diagrammatic supertraces along with the equivalent expressions that arise through this process (see [23] for details). Our notation in functional diagrams below is the following: heavy leptoquark fields $S_{i}$ with masses $M_{i}$ (double-dashed lines), $f, f^{\prime}, f^{\prime \prime}, f^{\prime \prime \prime}=q, u, d, \ell, e$ are the SM fermion fields (solid lines), $H$ is the SM Higgs-doublet (single dashed-lines), and $V=G, W, B$ are the SM gauge fields (wavy lines). Every circle indicates an insertion from $U$ (or in general $X$ )-matrices and $P_{\mu}$ is the covariant derivative. Furthermore, all SM fields are taken to be massless and $\eta^{\mu \nu}=(1,-1,-1,-1)$ is the metric tensor. We these definitions we obtain:

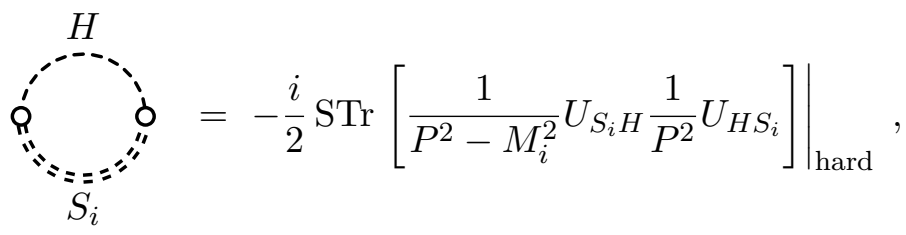




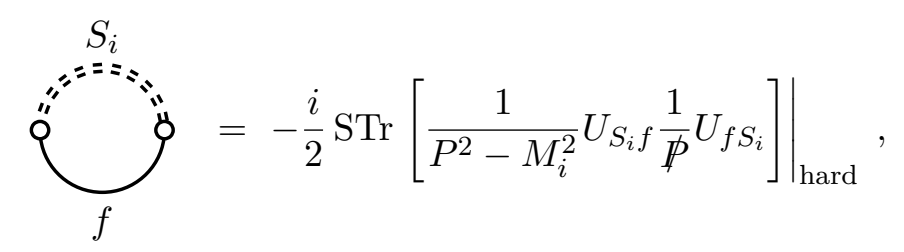

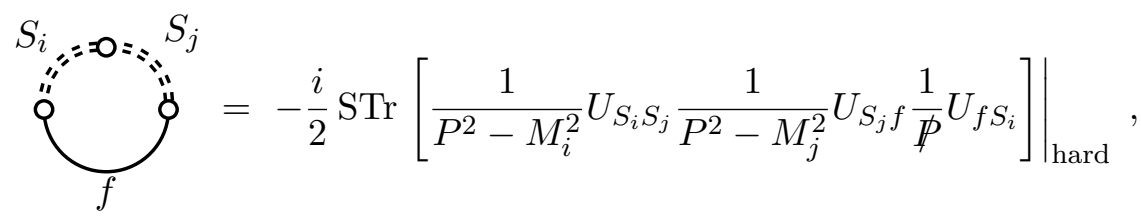

$\overbrace{S_{i} "==0}^{f} \oint_{f^{\prime}}=-\left.\frac{i}{2} \operatorname{STr}\left[\frac{1}{P^{2}-M_{i}^{2}} U_{S_{i} f} \frac{1}{\not P} U_{f f^{\prime}} \frac{1}{\not P} U_{f^{\prime} S_{i}}\right]\right|_{\text {hard }}$,

$$
\bigcap_{S_{i}=0}^{f=} \sum_{f^{\prime}}^{H}=-\left.\frac{i}{2} \mathrm{~S} \operatorname{Tr}\left[\frac{1}{P^{2}-M_{i}^{2}} U_{S_{i} f} \frac{1}{\not P} U_{f H} \frac{1}{P^{2}} U_{H f^{\prime}} \frac{1}{P} U_{f^{\prime} S_{i}}\right]\right|_{\text {hard }},
$$

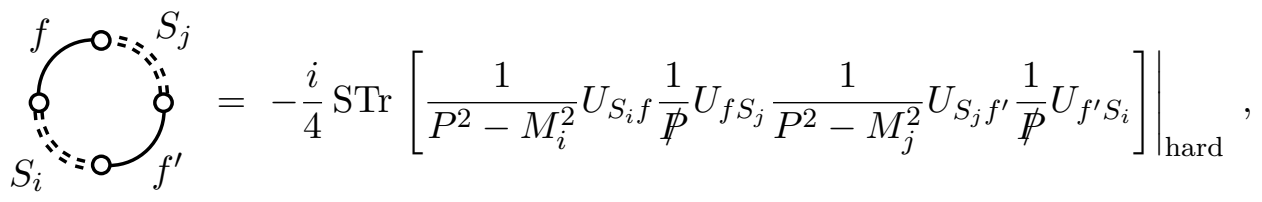

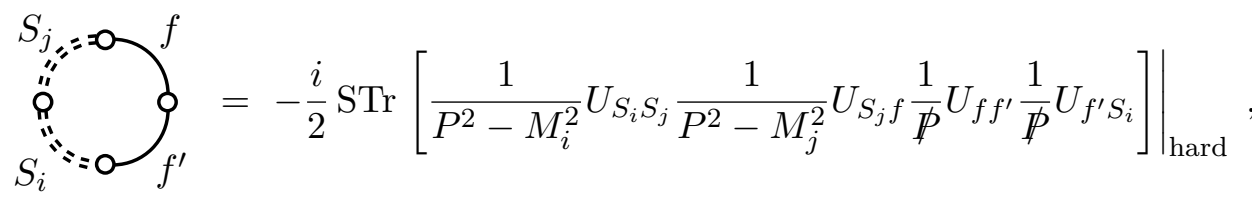

$$
\overbrace{S_{i}}^{f=} \sum_{f^{\prime}}^{\infty}=-\left.\frac{i}{2} \mathrm{~S} \operatorname{Tr}\left[\frac{1}{P^{2}-M_{i}^{2}} U_{S_{i} f} \frac{1}{\not P} U_{f V}^{\mu} \frac{-\eta_{\mu \nu}}{P^{2}} U_{V f^{\prime}}^{\nu} \frac{1}{P} U_{f^{\prime} S_{i}}\right]\right|_{\text {hard }},
$$

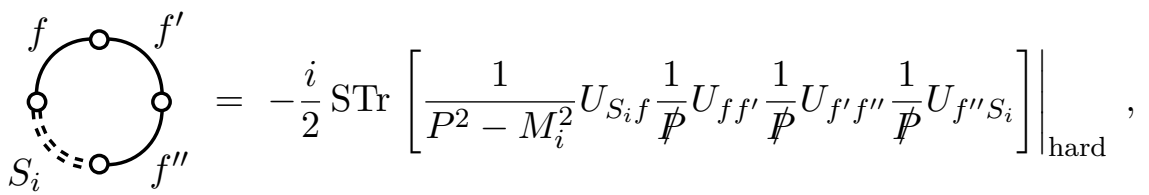

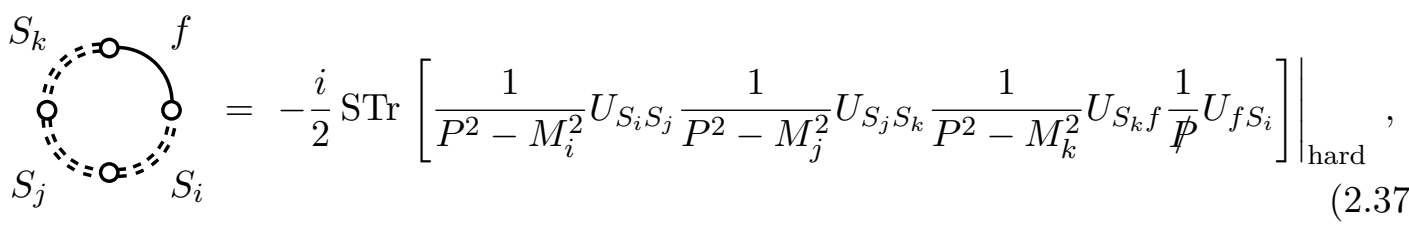


$f \overbrace{S_{i}=0}^{f^{\prime}} \int_{f^{\prime \prime \prime}}^{f^{\prime \prime}}=-\left.\frac{i}{2} \mathrm{STr}\left[\frac{1}{P^{2}-M_{i}^{2}} U_{S_{i}} \frac{1}{\not P} U_{f f^{\prime}} \frac{1}{\not P} U_{f^{\prime} f^{\prime \prime}} \frac{1}{\not P} U_{f^{\prime \prime} f^{\prime \prime \prime}} \frac{1}{\not P} U_{f^{\prime \prime \prime} S_{i}}\right]\right|_{\text {hard }}$

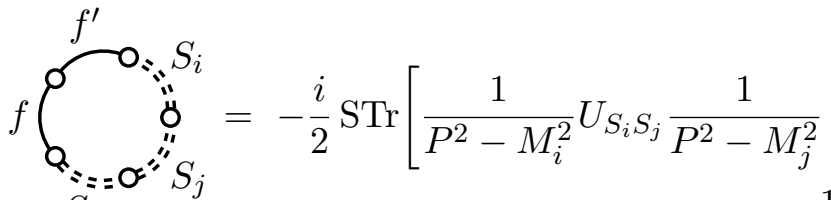

$$
\begin{aligned}
& \left.\times U_{S_{j} S_{k}} \frac{1}{P^{2}-M_{k}^{2}} U_{S_{k} f} \frac{1}{\not p} U_{f f^{\prime}} \frac{1}{\not P} U_{f^{\prime} S_{i}}\right]\left.\right|_{\text {hard }},
\end{aligned}
$$

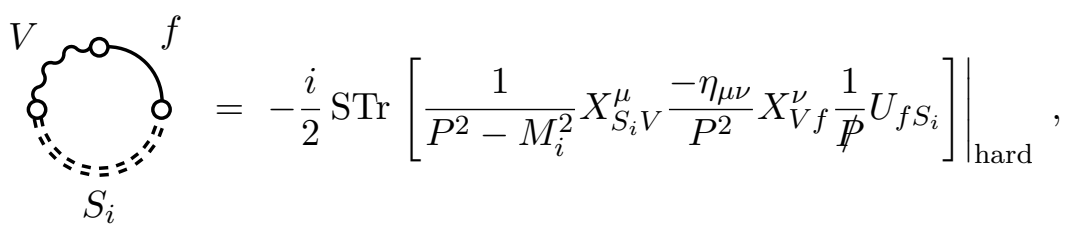

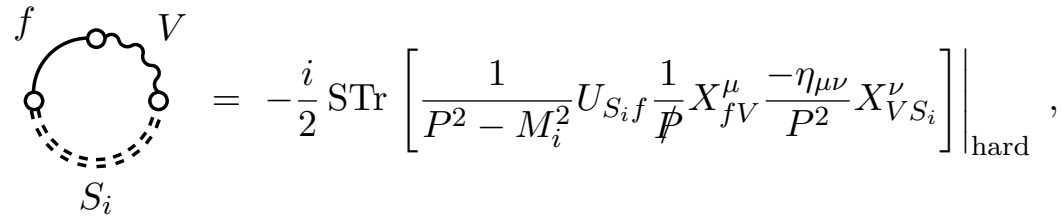

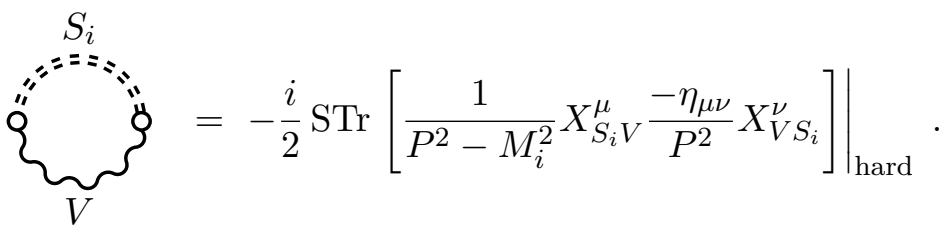

The total amount of heavy-light supertrace diagrams adds up to number 15 .

In appendix B one can find the explicit comparison between the number of covariant diagrams that match to a single Supertrace diagram. There the advantage of Supertraces is more evident.

\subsection{Evaluating $\mathcal{L}_{\text {EFT }}^{(1-\text { loop})}$}

The full 1-loop effective action is the sum of UOLEA for heavy-heavy loops and functional supertrace diagrams for heavy-light loops

$$
\mathcal{L}_{\mathrm{EFT}}^{(1-\text { loop })}=\mathcal{L}_{\mathrm{UOLEA}}+\mathcal{L}_{\mathrm{STr}},
$$

respectively. The UOLEA for only-heavy particles circulating in the loop, derived in ref. [10] and then re-derived in ref. [16], reads:

$$
\begin{aligned}
\mathcal{L}_{\text {UOLEA }}= & -i c_{s} \operatorname{tr}\left\{f_{2}^{S_{i}} U_{S_{i} S_{i}}+f_{3}^{S_{i}} G_{S_{i}}^{\prime \mu \nu} G_{\mu \nu, S_{i}}^{\prime}+f_{4}^{S_{i} S_{j}} U_{S_{i} S_{j}} U_{S_{j} S_{i}}\right. \\
& +f_{5}^{S_{i}}\left(P^{\mu} G_{\mu \nu, S_{i}}^{\prime}\right)\left(P_{\rho} G_{S_{i}}^{\rho \nu}\right)+f_{6}^{S_{i}} G_{\nu, S_{i}}^{\prime \mu} G_{\rho, S_{i}}^{\prime \nu} G_{\mu, S_{i}}^{\rho} \\
& +f_{7}^{S_{i} S_{j}}\left(P^{\mu} U_{S_{i} S_{j}}\right)\left(P_{\mu} U_{S_{j} S_{i}}\right)+f_{8}^{S_{i} S_{j} S_{k}} U_{S_{i} S_{j}} U_{S_{j} S_{k}} U_{S_{k} S_{i}}
\end{aligned}
$$




$$
\begin{aligned}
& +f_{9}^{S_{i}} U_{S_{i} S_{i}} G_{S_{i}}^{\prime \mu \nu} G_{\mu \nu, S_{i}}^{\prime}+f_{10}^{S_{i} S_{j} S_{k} S_{l}} U_{S_{i} S_{j}} U_{S_{j} S_{k}} U_{S_{k} S_{l}} S_{S_{l} S_{i}} \\
& +f_{11}^{S_{i} S_{j} S_{k}} U_{S_{i} S_{j}}\left(P_{\mu} U_{S_{j} S_{k}}\right)\left(P^{\mu} U_{S_{k} S_{i}}\right) \\
& +f_{12}^{S_{i} S_{j}}\left(P^{2} U_{S_{i} S_{j}}\right)\left(P^{2} U_{S_{j} S_{i}}\right)+f_{13}^{S_{i} S_{j}} U_{S_{i} S_{j}} U_{S_{j} S_{i}} G_{S_{i}}^{\prime \mu \nu} G_{\mu \nu, S_{i}}^{\prime} \\
& +f_{14}^{S_{i} S_{j}}\left(P_{\mu} U_{S_{i} S_{j}}\right)\left(P_{\nu} U_{S_{j} S_{i}}\right) G_{S_{i}}^{\prime \nu \mu} \\
& +f_{15}^{S_{i} S_{j}}\left(U_{S_{i} S_{j}}\left(P^{\mu} U_{S_{j} S_{i}}\right)-\left(P^{\mu} U_{S_{i} S_{j}}\right) U_{S_{j} S_{i}}\right)\left(P^{\nu} G_{\nu \mu, S_{i}}^{\prime}\right) \\
& +f_{16}^{S_{i} S_{j} S_{k} S_{l} S_{m}} U_{S_{i} S_{j}} U_{S_{j} S_{k}} U_{S_{k} S_{l}} U_{S_{l} S_{m}} U_{S_{m} S_{i}} \\
& +f_{17}^{S_{i} S_{j} S_{k} S_{l}} U_{S_{i} S_{j}} U_{S_{j} S_{k}}\left(P^{\mu} U_{S_{k} S_{l}}\right)\left(P_{\mu} U_{S_{l} S_{i}}\right) \\
& +f_{18}^{S_{i} S_{j} S_{k} S_{l}} U_{S_{i} S_{j}}\left(P^{\mu} U_{S_{j} S_{k}}\right) U_{S_{k} S_{l}}\left(P_{\mu} U_{S_{l} S_{i}}\right) \\
& \left.+f_{19}^{S_{i} S_{j} S_{k} S_{l} S_{m} S_{n}} U_{S_{i} S_{j}} U_{S_{j} S_{k}} U_{S_{k} S_{l}} U_{S_{l} S_{m}} U_{S_{m} S_{n}} U_{S_{n} S_{i}}\right\},
\end{aligned}
$$

where $G^{\prime}=-\left[P_{\mu}, P_{\nu}\right]=-i g G_{\mu \nu}$ and $g$ is the coupling of the corresponding field strength tensor $G_{\mu \nu} . P_{\mu}$ is the covariant derivative that act to the right in every parenthesis. To get the correct contribution for Wilson coefficients we multiply these terms with $\left(-i c_{s}\right)$. Since we separate each complex scalar into a two component field multiplet, each component counts as a real degree of freedom, thus the correct value is, $c_{s}=1 / 2$. We should note that after the term $f_{9}$ only the non-diagonal terms in $U_{S S}$ contribute and exclusively the mass dimension-1 terms of the Lagrangian. In this formula a summation over leptoquark fields $S_{i}$ from table 1 is implied. The expressions for the coefficients, $f_{1}, \ldots, f_{19}$, can be found in ref. [16]. Appropriate limits of these expressions must be taken in case of degeneracies (i.e. more than one single $S$-field in the loop). Note that only U-matrices, calculated with $S_{i}=S_{i, c}[\phi]$, appear in (2.44).

We now need to calculate $\mathcal{L}_{\mathrm{STr}}$ in $(2.43)$. For this purpose we use the package STrEAM [24] in order to calculate the relevant supertraces in (2.28)-(2.42). The main function of this package is the automation of the CDE application. As a result it computes local traces for further calculation inserting the explicit expressions of the $\mathbf{X}$-matrices. We note that the option No $\gamma$ inU removes all spinor indices from all matrices. However, in some instances the outer matrices contain spinor indices while the internal ones do not, or if they do, these matrices (anti)commute with $\gamma$-matrices. Therefore some manual intervention is necessary to obtain the final result.

The result of this procedure is given below. The traces are categorized depending on the number of $U$ 's and $Z$ 's involved in the respective diagram. The single term from the heavy-light UOLEA $[(2.28)]$, derived in refs. $[11,12]$, is also included here. The prefactor $-i c_{s}$ is omitted, $c_{s}=1 / 2$ for complex scalars, and note that the matrix $U_{f f}$ contains only chirality projection operators $P_{(L, R)}$, which have been taken into account while anticommuting $\gamma$-matrices. At the end, $\mathcal{L}_{\mathrm{STr}}$ in $(2.43)$ is obtained from the equation,

$$
\mathcal{L}_{\mathrm{STr}}=\left(-i c_{s}\right) \times(\text { sum of all contributions below }[\mathrm{cf} .(2.46)-(2.72)]) .
$$

$\mathcal{O}\left(\boldsymbol{U}^{2}\right)$ :

$$
\left(1+\log \frac{\mu^{2}}{M_{i}^{2}}\right) \operatorname{tr}\left\{U_{S_{i} H} U_{H S_{i}}\right\},
$$




$$
\begin{aligned}
\frac{1}{2}\left(\frac{1}{2}+\log \frac{\mu^{2}}{M_{i}^{2}}\right) \operatorname{tr}\left\{U_{S_{i} f} \gamma_{\mu}\left(P^{\mu} U_{f S_{i}}\right)\right\} \\
\frac{1}{12 M_{i}^{2}} \operatorname{tr}\left\{U_{S_{i} f} \gamma^{\mu}\left(P^{2} P_{\mu} U_{f S_{i}}\right)+U_{S_{i} f} \gamma^{\mu}\left(P_{\mu} P^{2} U_{f S_{i}}\right)\right\}, \\
-\frac{1}{18 M_{i}^{2}} \operatorname{tr}\left\{U_{S_{i} f} \gamma_{\nu} U_{f S_{i}}\left(P_{\mu} G_{S_{i}}^{\mu \nu}\right)\right\}, \\
-\frac{1}{3 M_{i}^{2}}\left(\frac{7}{12}+\log \frac{\mu^{2}}{M_{i}^{2}}\right) \operatorname{tr}\left\{U_{S_{i} f} \gamma_{\nu}\left(P^{\mu} G_{f}^{\prime \mu \nu}\right) U_{f S_{i}}\right\}, \\
\frac{i}{2 M_{i}^{2}} \operatorname{tr}\left\{\left(P^{\mu} U_{S_{i} f}\right) \tilde{G}_{\mu \nu}^{\prime} \gamma^{\nu} \gamma_{5} U_{f S_{i}}-U_{S_{i} f} \tilde{G}_{\mu \nu}^{\prime} \gamma^{\nu} \gamma_{5}\left(P^{\mu} U_{f S_{i}}\right)\right\} .
\end{aligned}
$$

$\mathcal{O}\left(\boldsymbol{U}^{\mathbf{3}}\right)$ :

$$
\begin{gathered}
\left(1+\log \frac{\mu^{2}}{M_{i}^{2}}\right) \operatorname{tr}\left\{U_{S_{i} f} U_{f f^{\prime}} U_{f^{\prime} S_{i}}\right\} \\
\frac{2 \Delta_{i j}^{2}+\left(M_{i}^{2}+M_{j}^{2}\right) \log M_{j}^{2} / M_{i}^{2}}{4\left(\Delta_{i j}^{2}\right)^{2}} \operatorname{tr}\left\{\left(P^{\mu} U_{S_{i} S_{j}}\right) U_{S_{j} f} \gamma_{\mu} U_{f S_{i}}\right\} \\
\frac{1}{4 \Delta_{i j}^{2}} \log \frac{M_{j}^{2}}{M_{i}^{2}} \operatorname{tr}\left\{U_{S_{i} S_{j}} U_{S_{j} f} \gamma_{\mu}\left(P^{\mu} U_{f S_{i}}\right)-U_{S_{i} S_{j}}\left(P^{\mu} U_{S_{j} f}\right) \gamma_{\mu} U_{f S_{i}}\right\} \\
-\frac{1}{2 M_{i}^{2}}\left(\frac{1}{2}+\log \frac{\mu^{2}}{M_{i}^{2}}\right) \operatorname{tr}\left\{U_{S_{i} f}\left(P^{2} U_{f f^{\prime}}\right) U_{f^{\prime} S_{i}}\right\} \\
\frac{1}{2 M_{i}^{2}} \operatorname{tr}\left\{U_{S_{i} f} U_{f f^{\prime}}\left(P^{2} U_{f^{\prime} S_{i}}\right)\right\} \\
-\frac{1}{4 M_{i}^{2}}\left(\frac{3}{2}+\log \frac{\mu^{2}}{M_{i}^{2}}\right) \operatorname{tr}\left\{U_{S_{i} f}\left(P_{\mu} U_{f f^{\prime}}\right)\left(P^{\mu} U_{f^{\prime} S_{i}}\right)\right\} \\
-\frac{1}{4 M_{i}^{2}}\left(\frac{1}{2}+\log \frac{\mu^{2}}{M_{i}^{2}}\right) \operatorname{tr}\left\{U_{f f^{\prime}} i \sigma_{\mu \nu} G_{f^{\prime}}^{\prime \mu \nu} U_{f^{\prime} S_{i}}\right\} \\
\frac{1}{2 M_{i}^{2}} \operatorname{tr}\left\{U_{S_{i} f} i \sigma_{\mu \nu}\left(P_{f}^{\mu} U_{f f^{\prime}} U_{f S_{i}}\right\}\right.
\end{gathered}
$$

$\mathcal{O}\left(\boldsymbol{U}^{4}\right)$ :

$$
\begin{gathered}
-\frac{1}{4 M_{i}^{2}}\left(\frac{3}{2}+\log \frac{\mu^{2}}{M_{i}^{2}}\right) \operatorname{tr}\left\{U_{S_{i} f} \gamma^{\mu} U_{f H} U_{H f^{\prime}} \gamma_{\mu} U_{f^{\prime} S_{i}}\right\}, \\
-\frac{\log M_{i}^{2} / M_{j}^{2}}{4 \Delta_{i j}^{2}} \operatorname{tr}\left\{U_{S_{i} f} \gamma^{\mu} U_{f S_{j}} U_{S_{j} f^{\prime}} \gamma_{\mu} U_{f^{\prime} S_{i}}\right\}, \\
-\frac{\log M_{i}^{2} / M_{j}^{2}}{\Delta_{i j}^{2}} \operatorname{tr}\left\{U_{S_{i} S_{j}} U_{S_{j} f} U_{f f^{\prime}} U_{f^{\prime} S_{i}}\right\} \\
-\frac{1}{4 M_{i}^{2}}\left(\frac{3}{2}+\log \frac{\mu^{2}}{M_{i}^{2}}\right) \operatorname{tr}\left\{U_{S_{i} f} \gamma^{\mu} U_{f V} U_{V f^{\prime}} \gamma_{\mu} U_{f^{\prime} S_{i}}\right\}
\end{gathered}
$$




$$
\begin{aligned}
& -\frac{1}{4 M_{i}^{2}} \operatorname{tr}\left\{U_{S_{i} f} U_{f f^{\prime}} U_{f^{\prime} f^{\prime \prime}} \gamma_{\mu}\left(P^{\mu} U_{f^{\prime \prime} S_{i}}\right)\right. \\
& \left.-\left(P^{\mu} U_{f S_{i}}\right) U_{f f^{\prime}} U_{f^{\prime} f^{\prime \prime}} \gamma_{\mu} U_{f^{\prime \prime} S_{i}}\right\}, \\
& -\frac{1}{2 M_{i}^{2}}\left(1+\log \frac{\mu^{2}}{M_{i}^{2}}\right) \operatorname{tr}\left\{U_{f S_{i}} U_{f f^{\prime}}\left(P^{\mu} U_{f^{\prime} f^{\prime \prime}}\right) \gamma_{\mu} U_{f^{\prime \prime} S_{i}}\right. \\
& \left.-U_{f S_{i}}\left(P^{\mu} U_{f f^{\prime}}\right) U_{f^{\prime} f^{\prime \prime}} \gamma_{\mu} U_{f^{\prime \prime} S_{i}}\right\}, \\
& \mathcal{I}\left[q^{2}\right]_{i j k 0}^{1112} \operatorname{tr}\left\{U_{S_{i} S_{j}} U_{S_{j} S_{k}} U_{S_{k} f} \gamma^{\mu}\left(P_{\mu} U_{f S_{i}}\right)\right. \\
& \left.-U_{S_{i} S_{j}} U_{S_{j} S_{k}}\left(P^{\mu} U_{S_{k} f}\right) \gamma_{\mu} U_{f S_{i}}\right\}, \\
& -\mathcal{I}\left[q^{2}\right]_{i j k 0}^{1211} \operatorname{tr}\left\{U_{S_{i} S_{j}}\left(P^{\mu} U_{S_{j} S_{k}}\right) U_{S_{k} f} \gamma_{\mu} U_{f S_{i}}\right. \\
& \left.-\left(P^{\mu} U_{S_{i} S_{j}}\right) U_{S_{j} S_{k}} U_{S_{k} f} \gamma_{\mu} U_{S_{i} f}\right\} .
\end{aligned}
$$

$\mathcal{O}\left(U^{5}\right):$

$$
\begin{array}{r}
\frac{1}{M_{i}^{2}}\left(1+\log \frac{\mu^{2}}{M_{i}^{2}}\right) \operatorname{tr}\left\{U_{S_{i} f} U_{f f^{\prime}} U_{f^{\prime} f^{\prime \prime}} U_{f^{\prime \prime} f^{\prime \prime \prime}} U_{f^{\prime \prime \prime} S_{i}}\right\} \\
4 \mathcal{I}\left[q^{2}\right]_{i j k 0}^{1112} \operatorname{tr}\left\{U_{S_{i} S_{j}} U_{S_{j} S_{k}} U_{S_{k} f} U_{f f^{\prime}} U_{f^{\prime} S_{i}}\right\}
\end{array}
$$

$\mathcal{O}\left(Z^{1}\right)$

$$
\frac{1}{4}\left(\frac{3}{2}+\log \frac{\mu^{2}}{M_{i}^{2}}\right) \operatorname{tr}\left\{Z_{S_{i} V}^{\mu} U_{V f} \gamma_{\mu} U_{f S_{i}}+U_{S_{i} f} \gamma_{\mu} U_{f V} \bar{Z}_{V S_{i}}^{\mu}\right\}
$$

$\mathcal{O}\left(Z^{2}\right)$

$$
\frac{M_{i}^{2}}{4}\left(\frac{3}{2}+\log \frac{\mu^{2}}{M_{i}^{2}}\right) \operatorname{tr}\left\{Z_{S_{i} V}^{\mu} \bar{Z}_{V S_{i}, \mu}\right\} .
$$

In eqs. (2.46)-(2.72) above, the trace (tr) stands for a normal trace over the product of matrices- $(U, Z)$ that are direct products of spinor, gauge or flavour matrices. Also, with $\widetilde{G}$ we denote the usual dual tensor $\widetilde{G}_{\mu \nu}^{\prime}=\frac{1}{2} \epsilon^{\mu \nu \rho \sigma} G_{\rho \sigma}^{\prime}$. The $\mu$-parameter denotes the renormalization scale and the $\overline{M S}$-renormalization scheme with dimensional regularization is used throughout. Finally, the expressions for few integrals appearing before traces are $\left(\Delta_{i j}^{2} \equiv M_{i}^{2}-M_{j}^{2}\right)$,

$$
\begin{aligned}
\mathcal{I}\left[q^{2}\right]_{i j k 0}^{1112}= & \frac{\log M_{k}^{2} / M_{i}^{2}}{4 \Delta_{i j}^{2} \Delta_{i k}^{2}}-\frac{\log M_{k}^{2} / M_{j}^{2}}{4 \Delta_{i j}^{2} \Delta_{j k}} \\
\mathcal{I}\left[q^{2}\right]_{i j k 0}^{1211}= & \frac{1}{4 \Delta_{i j}^{2}}\left(\frac{3}{2}+\frac{M_{i}^{2} \log \mu^{2} / M_{i}^{2}+M_{k}^{2} \log \mu^{2} / M_{k}^{2}}{\Delta_{i k}^{2}}\right) \\
& +\frac{\log \mu^{2} / M_{j}^{2}\left(M_{i}^{2} M_{k}^{2}-M_{j}^{4}\right)+M_{k}^{2} \log \mu^{2} / M_{k}^{2}\left(M_{i}^{2}-2 M_{j}^{2}+M_{k}^{2}\right)}{4\left(\Delta_{i j}^{2}\right)^{2}\left(\Delta_{j k}^{2}\right)^{2}} \\
& +\frac{2 M_{i}^{2}-5 M_{j}^{2}+3 M_{k}^{2}}{8\left(\Delta_{i j}^{2}\right)^{2} \Delta_{j k}^{2}} .
\end{aligned}
$$

Following this general procedure, the operators extracted from $\mathcal{L}_{\mathrm{EFT}}^{(1-\text { loop })}$ in $(2.43)$ are given in a general operator basis, usually referred to as Green basis, which does not involve 


\begin{tabular}{|c|ccc|}
\hline Field/Group & $\mathrm{SU}(3)$ & $\mathrm{SU}(2)$ & $\mathrm{U}(1)$ \\
\hline$S_{1}$ & $\overline{3}$ & 1 & $\frac{1}{3}$ \\
$\tilde{S}_{2}$ & 3 & 2 & $\frac{1}{6}$ \\
\hline
\end{tabular}

Table 2. Leptoquark charges under the SM gauge group for the $S_{1}+\tilde{S}_{2}$ model.

field EOMs in reducing the number operators, but only integration-by-parts. There is however, one more complication in writing down the Wilson coefficients even in Green basis. This is the appearance of the so-called evanescent operators $[45,46]$ that vanish in $d=4$ but do not vanish in general for $d \neq 4$ in certain four-fermion interactions. The effect of evanescent operators in SM EFT [33, 47, 48] is taken into account in the application we present in the next section.

It is easy to make the connection between eqs. (2.46)-(2.72) and the functional supertrace diagrams in eqs. (2.28)-(2.42). For example, say we want to find a diagram candidate for neutrino-mass generation operator $\ell \ell H H$. We need four interaction vertices, i.e. four $U$-matrices but no derivative $(P)$ operators or $\gamma$-matrices. Looking at $\mathcal{O}\left(U^{4}\right)$ terms we see that only (2.63) satisfies this condition. Following the subscripts of $U$-matrices, in this case $S_{i}-S_{j}-f-f^{\prime}-S_{i}$, we trivially see the corresponding diagram is that of (2.34). What is very nice in this approach is the fact that strict correlations between observables are now obvious, i.e., the operator resulting from (2.63) may be correlated with those containing the insertions $U_{S_{i} S_{j}}, U_{S_{i} f}$ and $U_{f f^{\prime}}$.

\subsection{Summary}

Our main formulae for the full 1-loop matching up-to dimension-6 order in EFT expansion for all scalar leptoquarks are:

1. Tree level matching: eq. (2.9),

2. One loop matching: eq. $(2.43)=$ eq. $(2.44)+$ eq. (2.45).

From now on we have to choose a specific model with heavy particles taken from table 1, plug in the explicit $\mathrm{X}(U, Z)$-matrices and operators will pop-out of the traces. The general form of interaction $\mathrm{X}(U, Z)$-matrices is presented in appendix $\mathrm{A}$.

\section{Application: the leptoquark model $S_{1}+\tilde{S}_{2}$}

In this section, we apply the machinery of functional matching onto a particular scalar leptoquark model. Consequently, we consider an extension of the SM consisting of two scalar colored leptoquarks, an isospin singlet and a doublet, $S_{1}$ and $\tilde{S}_{2}$, with masses $M_{1}$ and $\tilde{M}_{2}$, respectively. Their charges under the SM gauge group are shown in table 2.

Interestingly, $S_{1}$ and $\tilde{S}_{2}$ belong to irreducible representations of an $\mathrm{SU}(5)$ [or $\left.\mathrm{SO}(10)\right]$ Grand Unified Theory (GUT). For example, $S_{1}$ may belong to $\overline{\mathbf{5}}, \overline{\mathbf{4 5}}, \overline{\mathbf{5 0}}$ and $\tilde{S}_{2}$ to $\mathbf{1 0}, \mathbf{1 5}$ irreps of minimal $S U(5)$, respectively. From the SM EFT operator content we derive below, we see that this model predicts fast proton decay and neutrino masses (and related 
$B$ - and $L$-violating phenomena). Then it is natural for the two fields to have heavy masses $M_{i} \approx 10^{12} \mathrm{GeV}$ which at the same time control the proton decay rate and generate neutrino masses consistent with experimental constraints [49-52].

On the other hand, one may apply a baryon parity where lepton, quark and therefore leptoquark fields, transform differently under a symmetry in order to protect the model from proton decay (although such a symmetry is not in general natural in GUTs as we argue below). In this case, $M_{1}$ and $\tilde{M}_{2}$ may be within the few-TeV range. Again, one may be able to account for radiative neutrino masses [53, 54], or current anomalous events such as the muon anomalous magentic moment $[39,54-56]$ and certain $B$-meson decays [39, 40].

In either cases, this $S_{1}+\tilde{S}_{2}$-model seems to attract a certain phenomenological interest which motivates us for studying its effective operators and their matching onto the SM EFT Lagrangian. However, further than a functional matching demonstration, such as a detailed phenomenological consideration, are beyond the scope of this paper.

\subsection{Lagrangian and symmetries}

We split the leptoquark Lagrangian into three parts,

$$
\mathcal{L}_{\mathrm{BSM}}=\mathcal{L}_{\mathrm{S}-\mathrm{f}}+\mathcal{L}_{\mathrm{S}-\mathrm{H}}+\mathcal{L}_{\mathrm{S}}
$$

The first part refers to leptoquark-fermion interactions, the second one to leptoquark-Higgs interactions, while the last part contains self and mixed terms between the two leptoquarks. Explicitly the first part reads [33, 37],

$$
\begin{aligned}
\mathcal{L}_{\mathrm{S}-\mathrm{f}}= & {\left[\left(\lambda_{p r}^{1 \mathrm{~L}}\right) \bar{q}_{p i}^{c} \cdot \epsilon \cdot \ell_{r}+\left(\lambda_{p r}^{1 \mathrm{R}}\right) \bar{u}_{i}^{c} e_{r}\right] S_{1 i}+\text { h.c. } } \\
& +\left(\lambda_{p r}^{\not B L}\right) S_{1 i} \epsilon^{i j k} \bar{q}_{p j} \cdot \epsilon \cdot q_{r k}^{c}+\left(\lambda_{p r}^{\not B R}\right) S_{1 i} \epsilon^{i j k} \bar{d}_{p j} u_{r k}^{c}+\text { h.c. } \\
& +\left(\tilde{\lambda}_{p r}\right) \bar{d}_{p i} \tilde{S}_{2 i}^{T} \cdot \epsilon \cdot \ell_{r}+\text { h.c. }
\end{aligned}
$$

where $\ell$ and $q$ are the lepton and quark field $\mathrm{SU}(2)_{L}$-doublets while the singlets are denoted by $u, d, e$ in gauge basis ${ }^{5}$ and $\epsilon$ is the antisymmetric tensor with $\mathrm{SU}(2)_{L}$ indices. The matrix $\lambda_{p r}^{\not B L}$ is complex symmetric in flavor space while all other matrices in (3.2) are in general complex ones. From now on, we use the indices $p, r, s, t$ to denote flavor without making any distinction between quark and lepton flavors. We also use $i, j, k, l$ to label $\mathrm{SU}(3)$ fundamental indices, while the dot-product denotes $\mathrm{SU}(2)$ contractions in the fundamental representation. Later we will also use the letters $\alpha, \beta, \gamma, \delta$ for $\mathrm{SU}(2)$ fundamental and $I, J, K, L$ for $\mathrm{SU}(2)$ adjoint representation. Lastly, we use $A, B, C, D$ for the $\mathrm{SU}(3)$ adjoint representation and suppress spinor-indices throughout.

The next part of the Lagrangian, namely leptoquark-Higgs interactions, reads [41],

$$
\begin{aligned}
\mathcal{L}_{\mathrm{S}-\mathrm{H}}= & -\left(M_{1}^{2}+\lambda_{H 1}|H|^{2}\right)\left|S_{1}\right|^{2}-\left(\tilde{M}_{2}^{2}+\tilde{\lambda}_{H 2}|H|^{2}\right)\left|\tilde{S}_{2}\right|^{2}+\lambda_{\tilde{2} \tilde{2}}\left(\tilde{S}_{2 i}^{\dagger} \cdot H\right)\left(H^{\dagger} \cdot \tilde{S}_{2 i}\right) \\
& -A_{21}\left(\tilde{S}_{2 i}^{\dagger} \cdot H\right) S_{1 i}^{\dagger}+\frac{1}{3} \lambda_{3} \epsilon^{i j k}\left(\tilde{S}_{2 i}^{T} \cdot \epsilon \cdot \tilde{S}_{2 j}\right)\left(H^{\dagger} \cdot \tilde{S}_{2 k}\right)+\text { h.c. }
\end{aligned}
$$

\footnotetext{
${ }^{5}$ Field redefinitions and flavour rotations to mass-basis are performed following ref. [57] after running the SM EFT parameters down to the EW scale.
} 


\begin{tabular}{|c|c|c|c|}
\hline LQ-fields & $B$ & $L$ & $B-L$ \\
\hline$S_{1}$ & $-1 / 3$ & -1 & $2 / 3$ \\
\hline$\tilde{S}_{2}$ & $+1 / 3$ & -1 & $4 / 3$ \\
\hline Parameters & & & \\
\hline$\lambda^{\not B L}$ & +1 & +1 & 0 \\
\hline$\lambda^{\not B R}$ & +1 & +1 & 0 \\
\hline$A_{\tilde{2} 1}$ & 0 & -2 & 2 \\
\hline$A^{\prime}$ & -1 & 1 & -2 \\
\hline$\lambda_{3}$ & -1 & 3 & -4 \\
\hline
\end{tabular}

Table 3. $B, L$ and $B-L$ quantum numbers for the fields $S_{1}$ and $\tilde{S}_{2}$ and parameters (promoted to fields). For normalization we take $B(q)=1 / 3$ and $L(\ell)=1$. All other parameters in eqs. (3.2), (3.3) and (3.4) which are not quoted here, have zero $B$ and $L$ quantum numbers.

The last part containing leptoquark self-interactions is,

$$
\begin{aligned}
-\mathcal{L}_{\mathrm{S}}= & \frac{c_{1}}{2}\left(S_{1}^{\dagger} S_{1}\right)^{2}+\frac{\tilde{c}_{2}}{2}\left(\tilde{S}_{2}^{\dagger} \cdot \tilde{S}_{2}\right)^{2}+c_{1 \tilde{2}}^{(1)}\left(S_{1}^{\dagger} S_{1}\right)\left(\tilde{S}_{2}^{\dagger} \cdot \tilde{S}_{2}\right)+c_{1 \tilde{2}}^{(2)}\left(\tilde{S}_{2 \alpha}^{\dagger} S_{1}\right)\left(S_{1}^{\dagger} \tilde{S}_{2 \alpha}\right) \\
& +c_{\tilde{2}}^{(8)}\left(\tilde{S}_{2 i}^{\dagger} \cdot \tilde{S}_{2 j}\right)\left(\tilde{S}_{2 j}^{\dagger} \cdot \tilde{S}_{2 i}\right)+\left[A^{\prime} S_{1 i}^{\dagger} \epsilon^{i j k}\left(\tilde{S}_{2 j}^{T} \cdot \epsilon \cdot \tilde{S}_{2 k}\right)+\text { h.c. }\right]
\end{aligned}
$$

Our convention for the covariant derivative is,

$$
D_{\mu}=\partial_{\mu}-i g^{\prime} Y B_{\mu}-i g T^{I} W_{\mu}^{I}-i g_{s} T^{A} G_{\mu}^{A},
$$

where each $T$ represents the gauge-group generators of the corresponding representation of a generic field and $Y$ is its hypercharge. The field strength tensors for $\mathrm{U}(1)_{Y}, \mathrm{SU}(2)_{L}$ and $\mathrm{SU}(3)_{c}$ gauge-fields are respectively,

$$
\begin{aligned}
B_{\mu \nu} & =\partial_{\mu} B_{\nu}-\partial_{\nu} B_{\mu}, \\
W_{\mu \nu}^{I} & =\partial_{\mu} W_{\nu}-\partial_{\nu} W_{\mu}+g \epsilon^{I J K} W_{\mu}^{J} W_{\nu}^{K}, \\
G_{\mu \nu}^{A} & =\partial_{\mu} G_{\nu}-\partial_{\nu} G_{\mu}+g_{s} f^{A B C} G_{\mu}^{B} G_{\nu}^{C} .
\end{aligned}
$$

Finally, the SM Yukawa couplings are defined as,

$$
-\mathcal{L}_{\mathrm{Y}}=\left(y_{E}\right)_{p r} \bar{\ell}_{p} \cdot H e_{r}+\left(y_{U}\right)_{p r} \bar{q}_{p i} \cdot \epsilon \cdot H^{*} u_{r i}+\left(y_{D}\right)_{p r} \bar{q}_{p i} \cdot H d_{r i}+\text { h.c. }
$$

while the Higgs potential is,

$$
V_{H}=-m^{2}\left(H^{\dagger} \cdot H\right)+\frac{\lambda}{2}\left(H^{\dagger} H\right)^{2} .
$$

It is always instructive in a given Lagrangian to check upon global symmetries such as Baryon $(B)$ and Lepton $(L)$ number, which may be broken by certain interaction parameters. For the $S_{1}+\tilde{S}_{2}$ model these are given in table 3 .

Obviously, by assuming baryon and/or lepton number conservation we can eliminate all terms proportional to couplings $\left(\lambda^{\not B L}, \lambda^{\not B R}, A^{\prime}, \lambda_{3}\right)$ and/or $A_{\tilde{2} 1}$, respectively. However, 
baryon and lepton symmetries cannot be well-defined gauge symmetries of a GUT model since they lead to chiral anomalies. On the other hand however, in SO(10)-GUTs for example (and also in the $\mathrm{SM}), B-L$ is an anomaly free gauge symmetry and therefore we

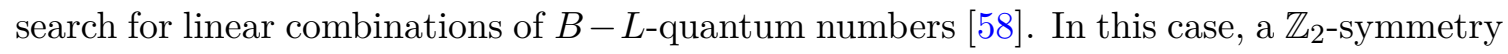
$\exp (2 \pi i(B-L) / 2)$, does not exclude any of couplings above, a $\mathbb{Z}_{3}$-symmetry $\exp (2 \pi i(B-$ $L) / 3$ ), excludes $A_{\tilde{2} 1}, A^{\prime}, \lambda_{3}$, a $\mathbb{Z}_{4}$-symmetry $\exp (2 \pi i(B-L) / 4)$, excludes $A_{\tilde{2} 1}, A^{\prime}$, which is not desirable if we want to generate neutrino masses by loop-corrections. Therefore, there is no $B-L$ discrete symmetry that rejects terms proportional to $\lambda^{\not B L}$ (and $\lambda^{\not B R}$ ) which lead to proton decay. We conclude that in general, an extra (and possibly ad-hoc) symmetry should be in order if we are about to pick a combination of leptoquark fields with masses nearby the TeV scale. For a recent discussion the reader is referred to ref. [59].

\subsection{Tree level matching}

As is the case in EFTs, we assume that both leptoquarks masses, $M_{i}=\left\{M_{1}, \tilde{M}_{2}\right\}$, are heavier than any other scale in the theory. Moreover, the parameters $A_{\tilde{2} 1}$, and $A^{\prime}$ should lay in region (2.4). To match at tree level we need the equations of motion (1.4), in order to derive the classical fields,

$$
\begin{aligned}
S_{1 i, c} & =\frac{1}{M_{1}^{2}}\left[-\left(\lambda_{p r}^{1 \mathrm{~L}}\right)^{\dagger} \bar{\ell}_{p} \cdot \epsilon \cdot q_{r i}^{c}+\left(\lambda_{p r}^{1 \mathrm{R}}\right)^{\dagger} \bar{e}_{p} u_{r i}^{c}+\left(\lambda_{p r}^{\not B L}\right)^{\dagger} \epsilon^{i j k} \bar{q}_{p j}^{c} \cdot \epsilon \cdot q_{r i}-\left(\lambda_{p r}^{\not B R}\right)^{\dagger} \epsilon^{i j k} \bar{u}_{p j}^{c} d_{r k}\right], \\
\tilde{S}_{2 i \alpha, c} & =\frac{1}{\tilde{M}_{2}^{2}}\left[-\left(\tilde{\lambda}_{p r}\right)^{\dagger}\left(\bar{\ell}_{p} \cdot \epsilon\right)_{\alpha} d_{r i}\right] .
\end{aligned}
$$

Substituting back into the Lagrangian [see (2.9)] we obtain the following Wilson coefficients that accompany $d=6$-operators. In what follows, we split the Wilson coefficients to tree and loop contributions as $G=G^{(0)}+\frac{1}{(4 \pi)^{2}} G^{(1)}$. The symbol $G$ denotes Wilson coefficients in Green basis, and we use exactly the same naming for operators as in ref. [33] that we append in appendix $\mathrm{C}$ for complementarity purposes.

In summary, we find the following twelve $B$-number conserving tree-level coefficients

$$
\begin{array}{ll}
{\left[G_{\ell q}^{(1)}\right]_{p r s t}^{(0)}=\frac{\left(\lambda_{s p}^{1 \mathrm{~L}}\right)^{*}\left(\lambda_{t r}^{1 \mathrm{~L}}\right)}{4 M_{1}^{2}},} & {\left[G_{\ell q}^{(3)}\right]_{p r s t}^{(0)}=-\frac{\left(\lambda_{s p}^{1 \mathrm{~L}}\right)^{*}\left(\lambda_{t r}^{1 \mathrm{~L}}\right)}{4 M_{1}^{2}},} \\
{\left[G_{l e q u}^{(1)}\right]_{p r s t}^{(0)}=\frac{\left(\lambda_{s p}^{1 \mathrm{~L}}\right)^{*}\left(\lambda_{t r}^{1 \mathrm{R}}\right)}{2 M_{1}^{2}},} & {\left[G_{\ell e q u}^{(3)}\right]_{p r s t}^{(0)}=-\frac{\left(\lambda_{s p}^{1 \mathrm{~L}}\right)^{*}\left(\lambda_{t r}^{1 \mathrm{R}}\right)}{8 M_{1}^{2}},} \\
{\left[G_{\text {eu }}\right]_{p r s t}^{(0)}=\frac{\left(\lambda_{s p}^{1 \mathrm{R}}\right)^{*}\left(\lambda_{t r}^{1 \mathrm{R}}\right)}{2 M_{1}^{2}},} & {\left[G_{\ell d}\right]_{p r s t}^{(0)}=-\frac{\left(\tilde{\lambda}_{t p}\right)^{*}\left(\tilde{\lambda}_{s r}\right)}{2 \tilde{M}_{2}^{2}},} \\
{\left[G_{q q}^{(1)}\right]_{p r s t}^{(0)}=\frac{\left(\lambda_{r t}^{\not B L}\right)^{*}\left(\lambda_{s p}^{\not B L}\right)}{2 M_{1}^{2}},} & {\left[G_{q q}^{(3)}\right]_{p r s t}^{(0)}=-\frac{\left(\lambda_{r t}^{\not B L}\right)^{*}\left(\lambda_{s p}^{\not B L}\right)}{2 M_{1}^{2}},} \\
{\left[G_{u d}^{(1)}\right]_{p r s t}^{(0)}=\frac{\left(\lambda_{t r}^{\not B R}\right)^{*}\left(\lambda_{s p}^{\not B R}\right)}{3 M_{1}^{2}},} & {\left[G_{u d}^{(8)}\right]_{p r s t}^{(0)}=-\frac{\left(\lambda_{t r}^{\not B R}\right)^{*}\left(\lambda_{s p}^{\not B R}\right)}{M_{1}^{2}},} \\
{\left[G_{q u q d}^{(1)}\right]_{p r s t}^{(0)}=\frac{4}{3} \frac{\left(\lambda_{t s}^{\not B R}\right)^{*}\left(\lambda_{p r}^{\not B L}\right)}{M_{1}^{2}},} & {\left[G_{q u q d}^{(8)}\right]_{p r s t}^{(0)}=-4 \frac{\left(\lambda_{t s}^{\not B R}\right)^{*}\left(\lambda_{p r}^{\not B L}\right)}{M_{1}^{2}},}
\end{array}
$$


and four $B$-number violating ones

$$
\begin{array}{ll}
{\left[G_{q q q}\right]_{p r s t}^{(0)}=-2 \frac{\left(\lambda_{p r}^{\not B L}\right)^{*}\left(\lambda_{s t}^{1 \mathrm{~L}}\right)}{M_{1}^{2}},} & {\left[G_{q q u}\right]_{p r s t}^{(0)}=\frac{\left(\lambda_{p r}^{\not B L}\right)^{*}\left(\lambda_{s t}^{1 \mathrm{R}}\right)}{M_{1}^{2}},} \\
{\left[G_{d u q}\right]_{p r s t}^{(0)}=\frac{\left(\lambda_{p r}^{\not B R}\right)^{*}\left(\lambda_{s t}^{1 \mathrm{~L}}\right)}{M_{1}^{2}},} & {\left[G_{d u u}\right]_{p r s t}^{(0)}=\frac{\left(\lambda_{p r}^{\not B R}\right)^{*}\left(\lambda_{s t}^{1 \mathrm{R}}\right)}{M_{1}^{2}} .}
\end{array}
$$

As we see all tree-level dimension-six operators are four-fermion operators in the effective Lagrangian. Besides $M_{i}$, their strength is governed by products of leptoquark Yukawa couplings.

Finally for completeness, we present the five tree-level $d=7$ Wilson coefficients in the basis of ref. [60]. They are,

$$
\begin{array}{rlrl}
{\left[G_{L L Q \bar{d} H}^{(1)}\right]_{p r s t}^{(0)}} & =-\frac{A_{\tilde{2} 1}}{M_{1}^{2} \tilde{M}_{2}^{2}}\left(\lambda_{s t}^{1 \mathrm{~L}}\right)\left(\tilde{\lambda}_{p r}\right), & & {\left[G_{L L Q \bar{d} H}^{(2)}\right]_{p r s t}^{(0)}=\frac{A_{\tilde{2} 1}}{M_{1}^{2} \tilde{M}_{2}^{2}}\left(\lambda_{s t}^{1 \mathrm{~L}}\right)\left(\tilde{\lambda}_{p r}\right),} \\
{\left[G_{\text {Leu } \bar{d} H}\right]_{p r s t}^{(0)}} & =\frac{A_{\tilde{2} 1}}{2 M_{1}^{2} \tilde{M}_{2}^{2}}\left(\lambda_{t r}^{1 \mathrm{R}}\right)\left(\tilde{\lambda}_{s p}\right), & & {\left[G_{L u d d H}\right]_{p r s t}^{(0)}=\frac{A_{\tilde{2} 1}^{*}}{M_{1}^{2} \tilde{M}_{2}^{2}}\left(\lambda_{s t}^{\not B R}\right)^{\dagger}\left(\tilde{\lambda}_{p r}\right)^{\dagger},} \\
{\left[G_{\bar{L} Q Q d H}\right]_{p r s t}^{(0)}} & =-2 \frac{A_{\tilde{2} 1}^{*}}{M_{1}^{2} \tilde{M}_{2}^{2}}\left(\lambda_{s t}^{\not B L}\right)^{*}\left(\tilde{\lambda}_{p r}\right)^{\dagger} . &
\end{array}
$$

As noted in the paragraph below eq. (2.9), coefficients associated with $d=7$ operators in eqs. (3.21)-(3.21) can be competitive to $d=6$ ones in (3.19)-(3.20) if there is a certain hierarchy between the two scales involved, e.g., $M_{1} \gg \tilde{M}_{2}$ and $A_{\tilde{2} 1} v / \tilde{M}_{2}^{2} \simeq 1$.

The appearance of products only $\lambda \cdot \lambda^{\prime}$ with $|\Delta(B-L)|=0$ and $A_{\tilde{2} 1} \lambda \cdot \lambda^{\prime}$ with $|\Delta(B-L)|=2$ in coefficients for $d=6$ and $d=7$ tree-level EFT operators respectively, is not accidental. It follows from the $B, L$-numbers for the parameters quoted in table 3 , and an interesting connection $[61,62]$ between $\Delta B$ and $\Delta L$ with the minimum and possible dimensionality of operators

$$
\begin{array}{rlrl}
d_{\min } & \geq \frac{9}{2}|\Delta B|+\frac{3}{2}|\Delta L|, & \\
|\Delta(B-L)| & =0,4,8,12,16, \ldots \quad & & (d-\text { even }), \\
|\Delta(B-L)| & =2,6,10,14,18, \ldots & & (d-\text { odd }) .
\end{array}
$$

For example, a coefficient proportional to $A_{\tilde{2} 1} \times(|\Delta(B-L)|=0$ couplings $)$ must necessarily be associated to odd-dimensional $d=3,5,7, \ldots$ case which is confirmed here at tree and below at one-loop level. Similarly, the dimension-full parameter $A^{\prime} \times(|\Delta(B-L)|=$ 0 couplings) will be associated for the first time with $d=7$-operators in EFT at 1-loop and at $d=9$ at tree level; the dimensionless parameter $\lambda_{3} \times(|\Delta(B-L)|=0$ couplings $)$ will appear first at $d=10$ and so on.

\subsection{One loop matching in the Green basis}

As we explained in section 2.3,one loop matching is carried out in two steps. First, the original heavy-only UOLEA in eq. (2.44) is used to derive operators with heavy leptoquark 
fields (for this model $S_{1}$ and $\tilde{S}_{2}$ ) circulating in the loop. Second, we use the general results from evaluating functional Supertraces in (2.45) to calculate Wilson coefficients involving both heavy and light fields in the loop.

We list all one-loop Wilson coefficients produced both from the UOLEA and the Supertraces. As before, we split them in tree and loop level coefficients as $G_{i}=G_{i}^{(0)}+\frac{1}{(4 \pi)^{2}} G_{i}^{(1)}$. Furthermore, for the quantities that renormalize $d=4$ operators we write, $\lambda^{\prime}=\lambda+\frac{1}{(4 \pi)^{2}} \delta \lambda$, $m^{\prime 2}=m^{2}+\frac{1}{(4 \pi)^{2}} \delta m^{2}, y_{n}^{\prime}=y_{n}+\frac{1}{(4 \pi)^{2}} \delta y_{n}$, where $n=E, U, D$ and the wave function renormalization is $Z_{k}=1+\frac{1}{(4 \pi)^{2}} \delta Z_{k}$ with $k=q, u, d, \ell, e$. For gauge bosons, we factor out of the resulting trace calculation the whole canonical kinetic term $-1 / 4 F_{\mu \nu} F^{\mu \nu}$, with $F_{\mu \nu}$ being a generic field strength tensor.Following an analogous naming scheme as in ref. [33] we define,

$$
L_{i}=\log \frac{\mu^{2}}{M_{i}^{2}}, \quad \text { with } \quad i=1,2,
$$

and the general $3 \times 3$ matrices,

$$
\begin{aligned}
& \Lambda_{\ell}=\left(\lambda^{1 \mathrm{~L}}\right)^{\dagger} \lambda^{1 \mathrm{~L}}, \\
& \Lambda_{u}=\left(\lambda^{1 \mathrm{R}}\right)^{*}\left(\lambda^{1 \mathrm{R}}\right)^{T}, \\
& \Lambda_{e}=\left(\lambda^{1 \mathrm{R}}\right)^{\dagger} \lambda^{1 \mathrm{R}}, \\
& \Lambda_{q}=\left(\lambda^{1 \mathrm{~L}}\right)^{*}\left(\lambda^{1 \mathrm{~L}}\right)^{T}, \\
& \tilde{\Lambda}_{d}=\tilde{\lambda} \tilde{\lambda}^{\dagger}, \\
& \tilde{\Lambda}_{\ell}=\tilde{\lambda}^{\dagger} \tilde{\lambda} \\
& \Lambda_{q}^{\not B}=\lambda^{\not B L}\left(\lambda^{\not B L}\right)^{\dagger}, \\
& \Lambda_{u}^{\not B}=\left(\lambda^{\not B R}\right)^{T}\left(\lambda^{\not B R}\right)^{*}, \\
& \Lambda_{d}^{\not B}=\lambda^{\not B R}\left(\lambda^{\not B R}\right)^{\dagger}, \\
& Y_{1 U}^{1 L}=\left(\lambda^{1 \mathrm{~L}}\right)^{\dagger} y_{U}^{*} \lambda^{1 \mathrm{R}}, \\
& Y_{1 E}^{1 L}=\left(\lambda^{1 \mathrm{R}} y_{E}^{\dagger}\left(\lambda^{1 \mathrm{~L}}\right)^{\dagger}\right)^{T}, \\
& Y_{2 U}^{1 L}=\left(\lambda^{1 \mathrm{~L}}\right)^{\dagger} y_{U}^{*} y_{U}^{T} \lambda^{1 \mathrm{~L}}, \\
& Y_{2 D}^{1 L}=\left(\lambda^{1 \mathrm{~L}}\right)^{\dagger} y_{D}^{*} y_{D}^{T} \lambda^{1 \mathrm{~L}} \text {, } \\
& Y_{2 U}^{1 R}=\left(\lambda^{1 \mathrm{R}}\right)^{\dagger} y_{U}^{T} y_{U}^{*} \lambda^{1 \mathrm{R}}, \\
& Y_{2 E}^{1 R}=\left(\lambda^{1 \mathrm{R}}\right)^{*} y_{E}^{T} y_{E}^{*}\left(\lambda^{1 \mathrm{R}}\right)^{T} \text {, } \\
& Y_{3 E}^{1 L}=\left(\lambda^{1 \mathrm{R}} y_{E}^{\dagger} y_{E} y_{E}^{\dagger}\left(\lambda^{1 \mathrm{~L}}\right)^{\dagger}\right)^{T} \\
& Y_{3 U}^{1 L}=\left(\lambda^{1 \mathrm{~L}}\right)^{\dagger} y_{U}^{*} y_{U}^{T} y_{U}^{*} \lambda^{1 \mathrm{R}}, \\
& \tilde{Y}_{2 E}=\tilde{\lambda} y_{E} y_{E}^{\dagger} \tilde{\lambda}^{\dagger}, \\
& \tilde{Y}_{2 D}=\tilde{\lambda}^{\dagger} y_{D}^{\dagger} y_{D} \tilde{\lambda}, \\
& Y_{1 D}^{\not B L}=\lambda^{\not B L} y_{D}^{*}\left(\lambda^{\not B R}\right)^{*}, \\
& Y_{1 U}^{\not B L}=\lambda^{\not B L} y_{U}^{*}\left(\lambda^{\not B R}\right)^{\dagger}, \\
& Y_{2 U}^{\not B R}=\left(\left(\lambda^{\not B R}\right)^{\dagger} y_{U}^{\dagger} y_{U} \lambda^{\not B R}\right)^{T}, \\
& Y_{2 D}^{\not B R}=\left(\left(\lambda^{\not B R}\right)^{\dagger} y_{D}^{\dagger} y_{D} \lambda^{\not B R}\right)^{T}, \\
& Y_{2 U}^{\not B L}=\left(\left(\lambda^{\not B L}\right)^{\dagger} y_{U} y_{U}^{\dagger} \lambda^{\not B L}\right)^{T}, \\
& Y_{2 D}^{\not B L}=\left(\left(\lambda^{\not B L}\right)^{\dagger} y_{D} y_{D}^{\dagger} \lambda^{\not B L}\right)^{T}, \\
& Y_{3 D}^{\not B L}=\left(\left(\lambda^{\not B R}\right)^{\dagger} y_{D}^{\dagger} y_{D} y_{D}^{\dagger} \lambda^{\not B L}\right)^{T}, \\
& Y_{3 U}^{\not B L}=\left(\lambda^{\not B R}\right)^{*} y_{U}^{\dagger} y_{U} y_{U}^{\dagger} \lambda^{\not B L} .
\end{aligned}
$$

Armed with those definitions we can now write the, lengthy but complete, one-loop Wilson coefficients associated to $d=4$ (renormalizable operators) all the way up-to $d=6$ operators in Green basis. All $d=5$ and 6 operators and the categories they belong to are given in appendix C. The hypercharges of $S_{1}$ and $\tilde{S}_{2}$ leptoquark fields are denoted as $Y_{S_{1}}$ and $Y_{\tilde{S}_{2}}$ respectively, and can be read from table 1. $N_{c}$ is the number of colours and $C_{F}^{G}$ is the quadratic Casimir of fundamental representation of group $G$. Finally, we define the squared mass difference quantity $\Delta_{12}^{2} \equiv M_{1}^{2}-\tilde{M}_{2}^{2}$. 


\subsubsection{Renormalizable operators}

$$
\begin{aligned}
& \left(\delta Z_{B}\right)=\frac{N_{c}}{3} g^{\prime 2}\left[Y_{S_{1}}^{2} L_{1}+2 Y_{\tilde{S}_{2}}^{2} L_{2}\right], \\
& \left(\delta Z_{W}\right)=\frac{N_{c}}{6} g^{2} L_{2}, \\
& \left(\delta Z_{G}\right)=\frac{N_{c}}{6} g_{s}^{2}\left[L_{1}+2 L_{2}\right], \\
& \left(\delta Z_{\ell}\right)_{p r}=\frac{N_{c}}{2}\left[\left(\frac{1}{2}+L_{1}\right)\left(\Lambda_{\ell}\right)_{p r}+\left(\frac{1}{2}+L_{2}\right)\left(\tilde{\Lambda}_{\ell}\right)_{p r}\right] \text {, } \\
& \left(\delta Z_{e}\right)_{p r}=\frac{N_{c}}{2}\left(\frac{1}{2}+L_{1}\right)\left(\Lambda_{e}\right)_{p r}, \\
& \left(\delta Z_{q}\right)_{p r}=\frac{1}{2}\left(\frac{1}{2}+L_{1}\right)\left(\Lambda_{q}-8 \Lambda_{q}^{\not B}\right)_{p r}, \\
& \left(\delta Z_{u}\right)_{p r}=\frac{1}{2}\left(\frac{1}{2}+L_{1}\right)\left(\Lambda_{u}+2 \Lambda_{u}^{\not B}\right)_{p r}, \\
& \left(\delta Z_{d}\right)_{p r}=\left(\frac{1}{2}+L_{2}\right)\left(\tilde{\Lambda}_{d}\right)_{p r}+\left(\frac{1}{2}+L_{1}\right)\left(\Lambda_{d}^{\not B}\right)_{p r}, \\
& \left(\delta Z_{H}\right)=N_{c}\left|A_{\tilde{2} 1}\right|^{2}\left[\frac{M_{1}^{2}+\tilde{M}_{2}^{2}}{2\left(\Delta_{12}^{2}\right)^{2}}+\frac{M_{1}^{2} \tilde{M}_{2}^{2} \log M_{1}^{2} / \tilde{M}_{2}^{2}}{\left(\Delta_{12}^{2}\right)^{3}}\right], \\
& \left(\delta y_{E}\right)_{p r}=-N_{c}\left(1+L_{1}\right)\left(Y_{1 U}^{1 L}\right)_{p r}, \\
& \left(\delta y_{U}\right)_{p r}=-N_{c}\left(1+L_{1}\right)\left(Y_{1 E}^{1 L}\right)_{p r}-4\left(Y_{1 D}^{B D L}\right)_{p r}\left(1+L_{1}\right) \text {, } \\
& \left(\delta y_{D}\right)_{p r}=-4\left(Y_{1 U}^{\not B L}\right)_{p r}\left(1+L_{1}\right) \text {, } \\
& (\delta \lambda)=N_{c}\left[\lambda_{H 1}^{2} L_{1}+\left(\tilde{\lambda}_{H 2}^{2}+\left(\tilde{\lambda}_{H 2}-\lambda_{\tilde{2} \tilde{2}}\right)^{2}\right) L_{2}\right. \\
& -\left|A_{\tilde{2} 1}\right|^{2} \lambda_{H 1} \frac{\Delta_{12}^{2}+\tilde{M}_{2}^{2} \log \tilde{M}_{2}^{2} / M_{1}^{2}}{\left(\Delta_{12}^{2}\right)^{2}} \\
& +\left|A_{\tilde{2} 1}\right|^{2}\left(\tilde{\lambda}_{H 2}-\lambda_{\tilde{2} \tilde{2}}\right) \frac{\Delta_{12}^{2}+M_{1}^{2} \log \tilde{M}_{2}^{2} / M_{1}^{2}}{\left(\Delta_{12}^{2}\right)^{2}} \\
& \left.-\frac{1}{2}\left|A_{\tilde{2} 1}\right|^{4} \frac{2 \Delta_{12}^{2}+\left(M_{1}^{2}+\tilde{M}_{2}^{2}\right) \log \tilde{M}_{2}^{2} / M_{1}^{2}}{\left(\Delta_{12}^{2}\right)^{3}}\right], \\
& \left(\delta m^{2}\right)=N_{c}\left[\lambda_{H 1} M_{1}^{2}\left(1+L_{1}\right)+\left(2 \tilde{\lambda}_{H 2}-\lambda_{\tilde{2} \tilde{2}}\right) \tilde{M}_{2}^{2}\left(1+L_{2}\right)\right. \\
& \left.+\left|A_{\tilde{2} 1}\right|^{2}\left(1+\frac{M_{1}^{2} \log \mu^{2} / M_{1}^{2}-\tilde{M}_{2}^{2} \log \mu^{2} / \tilde{M}_{2}^{2}}{\Delta_{12}^{2}}\right)\right] .
\end{aligned}
$$

\subsubsection{Dimension-5 operator}

$$
\left[G_{\nu \nu}\right]_{p r}^{(1)}=N_{c} A_{\tilde{2} 1}\left(\left(\lambda^{1 \mathrm{~L}}\right)^{T} y_{D} \tilde{\lambda}\right)_{p r} \frac{\log M_{1}^{2} / \tilde{M}_{2}^{2}}{M_{1}^{2}-\tilde{M}_{2}^{2}}
$$




\subsubsection{Vector bosons-scalar operators}

$X^{3}$ :

$$
\begin{aligned}
G_{3 G}^{(1)} & =\frac{g_{s}^{3}}{360}\left(\frac{1}{M_{1}^{2}}+\frac{2}{\tilde{M}_{2}^{2}}\right), \\
G_{3 W}^{(1)} & =\frac{g^{3} N_{c}}{360} \frac{1}{\tilde{M}_{2}^{2}} .
\end{aligned}
$$

$X^{2} D^{2}$ :

$$
\begin{aligned}
G_{2 B}^{(1)} & =\frac{N_{c}}{30} g^{\prime 2}\left(\frac{Y_{S_{1}}^{2}}{M_{1}^{2}}+2 \frac{Y_{\tilde{S}_{2}}^{2}}{\tilde{M}_{2}^{2}}\right), \\
G_{2 W}^{(1)} & =\frac{N_{c}}{60} g^{2} \frac{1}{\tilde{M}_{2}^{2}}, \\
G_{2 G}^{(1)} & =\frac{1}{60} g_{s}^{2}\left(\frac{1}{M_{1}^{2}}+\frac{2}{\tilde{M}_{2}^{2}}\right) .
\end{aligned}
$$

$X^{2} H^{2}$ :

$$
\begin{aligned}
& G_{H B}^{(1)}=N_{c} g^{\prime 2}\left[Y_{S_{1}}^{2}\left(\frac{\lambda_{H 1}}{12 M_{1}^{2}}-\left|A_{\tilde{2} 1}\right|^{2} \tilde{f}_{13}^{S_{1} \tilde{S}_{2}}\right)+Y_{\tilde{S}_{2}}^{2}\left(\frac{\left(2 \tilde{\lambda}_{H 2}-\lambda_{\tilde{2} \tilde{2}}\right)}{12 \tilde{M}_{2}^{2}}-\left|A_{\tilde{2} 1}\right|^{2} \tilde{f}_{13}^{\tilde{S}_{2} S_{1}}\right)\right], \\
& G_{H W}^{(1)}=N_{c} \frac{g^{2}}{4}\left(\frac{\left(\tilde{\lambda}_{H 2}-\lambda_{\tilde{2} \tilde{2}}\right)}{6 \tilde{M}_{2}^{2}}-\left|A_{\tilde{2} 1}\right|^{2} \tilde{f}_{13}^{\tilde{S}_{2} S_{1}}\right) \\
& G_{H G}^{(1)}=\frac{g_{s}^{2}}{2}\left[\frac{\lambda_{H 1}}{12 M_{1}^{2}}+\frac{\left(2 \tilde{\lambda}_{H 2}-\lambda_{\tilde{2} \tilde{2}}\right)}{12 \tilde{M}_{2}^{2}}-\left|A_{21}\right|^{2}\left(\tilde{f}_{13}^{S_{1} \tilde{S}_{2}}+\tilde{f}_{13}^{\tilde{S}_{2} S_{1}}\right)\right], \\
& G_{H W B}^{(1)}=-N_{c} g g^{\prime}\left[\left|A_{\tilde{2} 1}\right|^{2} \tilde{f}_{13}^{\tilde{S}_{2} S_{1}}+Y_{\tilde{S}_{2}} \frac{\lambda_{\tilde{2} \tilde{2}}}{12 \tilde{M}_{2}^{2}}\right] \text {. } \\
& G_{B D H}^{(1)}=N_{c}\left|A_{\tilde{2} 1}\right|^{2} g^{\prime}\left(Y_{S_{1}}+Y_{\tilde{S}_{2}}\right) \tilde{f}_{14}^{S_{1} \tilde{S}_{2}}, \\
& G_{W D H}^{(1)}=N_{c}\left|A_{\tilde{2} 1}\right|^{2} \frac{g}{2} \tilde{f}_{14}^{\tilde{S}_{2} S_{1}} \text {. }
\end{aligned}
$$

$H^{2} D^{4}:$

$$
G_{D H}^{(1)}=2 N_{c}\left|A_{\tilde{2} 1}\right|^{2} \tilde{f}_{12}^{S_{1}} \tilde{S}_{2} .
$$

$H^{4} D^{2}:$

$$
\begin{aligned}
G_{H \square}^{(1)} & =-\frac{N_{c}}{12}\left(\frac{\lambda_{H 1}^{2}}{M_{1}^{2}}+\frac{\tilde{\lambda}_{H 2}^{2}}{\tilde{M}_{2}^{2}}\right), \\
G_{H D}^{(1)} & =-N_{c} \frac{\lambda_{\tilde{2} \tilde{2}}}{6 \tilde{M}_{2}^{2}}+N_{c} \lambda_{\tilde{2} \tilde{2}}\left|A_{\tilde{2} 1}\right|^{2}\left(\tilde{f}_{11}^{\tilde{S}_{2} \tilde{S}_{2} S_{1}}-2 \tilde{f}_{11}^{S_{1} \tilde{S}_{2} \tilde{S}_{2}}\right)-N_{c}\left|A_{\tilde{2} 1}\right|^{4}\left(\tilde{f}_{17}^{\tilde{S}_{2} S_{1}}-2 \tilde{f}_{18}^{S_{1} \tilde{S}_{2}}\right),
\end{aligned}
$$




$$
\begin{aligned}
& G_{H D}^{\prime(1)}=-N_{c}\left|A_{\tilde{2} 1}\right|^{2}\left(\lambda_{H 1} \tilde{f}_{11}^{S_{1} S_{1} \tilde{S}_{2}}+\tilde{\lambda}_{H 2} \tilde{f}_{11}^{\tilde{S}_{2} \tilde{S}_{2} S_{1}}-2 \lambda_{\tilde{2} \tilde{2}} \tilde{f}_{11}^{S_{1} \tilde{S}_{2} \tilde{S}_{2}}\right)-N_{c}\left|A_{\tilde{2} 1}\right|^{4} \tilde{f}_{17}^{S_{1} \tilde{S}_{2}} \\
& G_{H D}^{\prime \prime(1)}=-i N_{c}\left|A_{\tilde{2} 1}\right|^{2}\left(\lambda_{H 1} \tilde{f}_{11}^{S_{1} \tilde{S}_{2} S_{1}}+\tilde{\lambda}_{H 2} \tilde{f}_{11}^{\tilde{S}_{2} S_{1} \tilde{S}_{2}}-\lambda_{\tilde{2} \tilde{2}} \tilde{f}_{11}^{S_{1} \tilde{S}_{2} \tilde{S}_{2}}\right)-i N_{c}\left|A_{\tilde{2} 1}\right|^{4} \tilde{f}_{18}^{S_{1} \tilde{S}_{2}} \\
& -\frac{i N_{c}}{12 \tilde{M}_{2}^{2}}\left(2 \tilde{\lambda}_{H 2} \lambda_{\tilde{2} \tilde{2}}-\lambda_{\tilde{2} \tilde{2}}^{2}\right) \text {. }
\end{aligned}
$$

$H^{6}$ :

$$
G_{H}^{(1)}=-\frac{N_{c}}{6}\left(\frac{\lambda_{H 1}^{3}}{M_{1}^{2}}+2 \frac{\tilde{\lambda}_{H 2}^{3}}{\tilde{M}_{2}^{2}}\right)+2 N_{c}\left|A_{\tilde{2} 1}\right|^{6} \tilde{f}_{19}^{S_{1} \tilde{S}_{2}} .
$$

\subsubsection{Two fermion operators}

$\psi^{2} D^{3}$ :

$$
\begin{aligned}
& {\left[G_{\ell D}\right]_{p r}^{(1)}=-\frac{N_{c}}{6}\left[\frac{\left(\Lambda_{\ell}\right)_{p r}}{M_{1}^{2}}+\frac{\left(\tilde{\Lambda}_{\ell}\right)_{p r}}{\tilde{M}_{2}^{2}}\right],} \\
& {\left[G_{e D}\right]_{p r}^{(1)}=-\frac{N_{c}}{6} \frac{\left(\Lambda_{e}\right)_{p r}}{M_{1}^{2}}} \\
& {\left[G_{q D}\right]_{p r}^{(1)}=-\frac{1}{6 M_{1}^{2}}\left[\left(\Lambda_{q}\right)_{p r}-8\left(\Lambda_{q}^{\not B}\right)_{p r}\right],} \\
& {\left[G_{u D}\right]_{p r}^{(1)}=-\frac{1}{6 M_{1}^{2}}\left[2\left(\Lambda_{u}^{\not B}\right)_{p r}+\left(\Lambda_{u}\right)_{p r}\right],} \\
& {\left[G_{d D}\right]_{p r}^{(1)}=\frac{\left(\tilde{\Lambda}_{d}\right)_{p r}}{3 \tilde{M}_{2}^{2}}-\frac{\left(\Lambda_{d}^{\not B}\right)_{p r}}{3 M_{1}^{2}}}
\end{aligned}
$$

$\psi^{2} X D$

$$
\begin{aligned}
& {\left[G_{W \ell}\right]_{p r}^{(1)}=-\frac{N_{c}}{6} g\left[\left(\frac{7}{12}+L_{1}\right) \frac{\left(\Lambda_{\ell}\right)_{p r}}{M_{1}^{2}}+\frac{\left(\tilde{\Lambda}_{\ell}\right)_{p r}}{6 \tilde{M}_{2}^{2}}\right]} \\
& {\left[G_{\widetilde{W} \ell}^{\prime}\right]_{p r}^{(1)}=\frac{N_{c}}{4} g \frac{\left(\Lambda_{\ell}\right)_{p r}}{M_{1}^{2}}} \\
& {\left[G_{B \ell}\right]_{p r}^{(1)}=\frac{N_{c}}{3} g^{\prime}\left[\left(\frac{7 Y_{q}-2 Y_{S_{1}}}{12}+Y_{q} L_{1}\right) \frac{\left(\Lambda_{\ell}\right)_{p r}}{M_{1}^{2}}-\left(\frac{7 Y_{d}+2 Y_{\tilde{S}_{2}}}{12}+Y_{d} L_{2}\right) \frac{\left(\tilde{\Lambda}_{\ell}\right)_{p r}}{\tilde{M}_{2}^{2}}\right]} \\
& {\left[G_{\widetilde{B} \ell}^{\prime}\right]_{p r}^{(1)}=-\frac{N_{c}}{2} g^{\prime}\left[Y_{q} \frac{\left(\Lambda_{\ell}\right)_{p r}}{M_{1}^{2}}-Y_{d} \frac{\left(\tilde{\Lambda}_{\ell}\right)_{p r}}{\tilde{M}_{2}^{2}}\right]} \\
& {\left[G_{B e}\right]_{p r}^{(1)}=\frac{N_{c}}{3} g^{\prime}\left(\frac{7 Y_{u}-2 Y_{S_{1}}}{12}+Y_{u} L_{1}\right) \frac{\left(\Lambda_{e}\right)_{p r}}{M_{1}^{2}}} \\
& {\left[G_{\widetilde{B} e}^{\prime}\right]_{p r}^{(1)}=\frac{N_{c}}{2} g^{\prime} Y_{u} \frac{\left(\Lambda_{e}\right)_{p r}}{M_{1}^{2}}} \\
& {\left[G_{G q}\right]_{p r}^{(1)}=\frac{1}{18} g_{s} \frac{\left(\Lambda_{q}\right)_{p r}}{M_{1}^{2}}-\frac{4}{3} g_{s} \frac{\left(\Lambda_{q}^{\not B}\right)_{p r}}{M_{1}^{2}}\left(\frac{3}{4}+L_{1}\right)} \\
& {\left[G_{\widetilde{G} q}^{\prime}\right]_{p r}^{(1)}=-2 g_{s} \frac{\left(\Lambda_{q}^{\not B}\right)_{p r}}{M_{1}^{2}}}
\end{aligned}
$$




$$
\begin{aligned}
& {\left[G_{W q}\right]_{p r}^{(1)}=-\frac{1}{6} g\left(\frac{7}{12}+L_{1}\right) \frac{\left(\Lambda_{q}\right)_{p r}+8\left(\Lambda_{q}^{\not B}\right)_{p r}}{M_{1}^{2}},} \\
& {\left[G_{\widetilde{W} q}^{\prime}\right]_{p r}^{(1)}=\frac{1}{4} g \frac{\left(\Lambda_{q}\right)_{p r}-8\left(\Lambda_{q}^{\not B}\right)_{p r}}{M_{1}^{2}}} \\
& {\left[G_{B q}\right]_{p r}^{(1)}=\frac{1}{3} g^{\prime}\left[\left(\frac{7 Y_{\ell}-2 Y_{S_{1}}}{12}+Y_{\ell} L_{1}\right) \frac{\left(\Lambda_{q}\right)_{p r}}{M_{1}^{2}}+\left(\frac{7 Y_{q}+2 Y_{S_{1}}}{12}+Y_{q} L_{1}\right) \frac{8\left(\Lambda_{q}^{\not B}\right)_{p r}}{M_{1}^{2}}\right] \text {, }} \\
& {\left[G_{\widetilde{B} q}^{\prime}\right]_{p r}^{(1)}=-\frac{1}{2} g^{\prime}\left[Y_{\ell} \frac{\left(\Lambda_{\ell}\right)_{p r}}{M_{1}^{2}}+Y_{q} \frac{8\left(\Lambda_{q}^{\not B}\right)_{p r}}{M_{1}^{2}}\right],} \\
& {\left[G_{G u}\right]_{p r}^{(1)}=\frac{1}{18} g_{s} \frac{\left(\Lambda_{u}\right)_{p r}}{M_{1}^{2}}-\frac{1}{3} g_{s}\left(\frac{3}{4}+L_{1}\right) \frac{\left(\Lambda_{u}^{\not B}\right)_{p r}}{M_{1}^{2}},} \\
& {\left[G_{\widetilde{G} u}^{\prime}\right]_{p r}^{(1)}=-\frac{1}{2} g_{s} \frac{\left(\Lambda_{u}^{\not B}\right)_{p r}}{M_{1}^{2}},} \\
& {\left[G_{B u}\right]_{p r}^{(1)}=\frac{1}{3} g^{\prime}\left[\left(\frac{7 Y_{e}-2 Y_{S_{1}}}{12}+Y_{e} L_{1}\right) \frac{\left(\Lambda_{u}\right)_{p r}}{M_{1}^{2}}+\left(\frac{7 Y_{d}+2 Y_{S_{1}}}{12}+Y_{d} L_{1}\right) \frac{2\left(\Lambda_{u}^{\not B}\right)_{p r}}{M_{1}^{2}}\right] \text {, }} \\
& {\left[G_{\widetilde{B} u}^{\prime}\right]_{p r}^{(1)}=\frac{1}{2} g^{\prime}\left[Y_{e} \frac{\left(\Lambda_{u}\right)_{p r}}{M_{1}^{2}}+Y_{d} \frac{2\left(\Lambda_{u}^{\not B}\right)_{p r}}{M_{1}^{2}}\right],} \\
& {\left[G_{G d}\right]_{p r}^{(1)}=\frac{1}{9} g_{s} \frac{\left(\tilde{\Lambda}_{d}\right)_{p r}}{\tilde{M}_{2}^{2}}-\frac{1}{3} g_{s}\left(\frac{3}{4}+L_{1}\right) \frac{\left(\Lambda_{d}^{\not B}\right)_{p r}}{M_{1}^{2}},} \\
& {\left[G_{\widetilde{G} d}^{\prime}\right]_{p r}^{(1)}=-\frac{1}{2} g_{s} \frac{\left(\Lambda_{d}^{\not B}\right)_{p r}}{M_{1}^{2}},} \\
& {\left[G_{B d}\right]_{p r}^{(1)}=-\frac{2}{3} g^{\prime}\left[\left(\frac{7 Y_{\ell}-2 Y_{S_{1}}}{12}+Y_{\ell} L_{1}\right) \frac{\left(\tilde{\Lambda}_{d}\right)_{p r}}{\tilde{M}_{2}^{2}}-\left(\frac{7 Y_{u}+2 Y_{S_{1}}}{12}+Y_{u} L_{1}\right) \frac{\left(\Lambda_{d}^{\not B}\right)_{p r}}{M_{1}^{2}}\right] \text {, }} \\
& {\left[G_{\widetilde{B} d}^{\prime}\right]_{p r}^{(1)}=g^{\prime}\left[2 Y_{\ell} \frac{\left(\tilde{\Lambda}_{d}\right)_{p r}}{\tilde{M}_{2}^{2}}+Y_{u} \frac{\left(\Lambda_{d}^{\not B}\right)_{p r}}{M_{1}^{2}}\right],} \\
& {\left[G_{G q}^{\prime}\right]_{p r}^{(1)}=\left[G_{W q}^{\prime}\right]_{p r}^{(1)}=\left[G_{B q}^{\prime}\right]_{p r}^{(1)}=0} \\
& {\left[G_{G u}^{\prime}\right]_{p r}^{(1)}=\left[G_{B u}^{\prime}\right]_{p r}^{(1)}=0 \text {, }} \\
& {\left[G_{G d}^{\prime}\right]_{p r}^{(1)}=\left[G_{B d}^{\prime}\right]_{p r}^{(1)}=0} \\
& {\left[G_{W \ell}^{\prime}\right]_{p r}^{(1)}=\left[G_{B \ell}^{\prime}\right]_{p r}^{(1)}=\left[G_{B e}^{\prime}\right]_{p r}^{(1)}=0 .}
\end{aligned}
$$

$\psi^{2} H D^{2}$ :

$$
\begin{aligned}
{\left[G_{e H D 1}\right]_{p r}^{(1)} } & =\frac{N_{c}}{2}\left(\frac{1}{2}+L_{1}\right) \frac{\left(Y_{1 U}^{1 L}\right)_{p r}}{M_{1}^{2}}, \\
{\left[G_{e H D 2}\right]_{p r}^{(1)} } & =+\frac{N_{c}}{2} \frac{\left(Y_{1 U}^{1 L}\right)_{p r}}{M_{1}^{2}},
\end{aligned}
$$




$$
\begin{aligned}
& {\left[G_{e H D 3}\right]_{p r}^{(1)}=-\frac{N_{c}}{2} \frac{\left(Y_{1 U}^{1 L}\right)_{p r}}{M_{1}^{2}}} \\
& {\left[G_{e H D 4}\right]_{p r}^{(1)}=-\frac{N_{c}}{2} \frac{\left(Y_{1 U}^{1 L}\right)_{p r}}{M_{1}^{2}}} \\
& {\left[G_{u H D 1}\right]_{p r}^{(1)}=\frac{1}{2}\left(\frac{1}{2}+L_{1}\right) \frac{\left(Y_{1 E}^{1 L}\right)_{p r}-4\left(Y_{1 D}^{\not B L}\right)_{p r}}{M_{1}^{2}}} \\
& {\left[G_{u H D 2}\right]_{p r}^{(1)}=\frac{\left(Y_{1 E}^{1 L}\right)_{p r}-4\left(Y_{1 D}^{\not B L}\right)_{p r}}{2 M_{1}^{2}}} \\
& {\left[G_{u H D 3}\right]_{p r}^{(1)}=-\frac{\left(Y_{1 E}^{1 L}\right)_{p r}-4\left(Y_{1 D}^{\not B L}\right)_{p r}}{2 M_{1}^{2}}} \\
& {\left[G_{u H D 4}\right]_{p r}^{(1)}=-\frac{\left(Y_{1 E}^{1 L}\right)_{p r}-4\left(Y_{1 D}^{\not B L}\right)_{p r}}{2 M_{1}^{2}}} \\
& {\left[G_{d H D 1}\right]_{p r}^{(1)}=-\frac{2}{M_{1}^{2}}\left(\frac{1}{2}+L_{1}\right)\left(Y_{1 U}^{\not B L}\right)_{p r}} \\
& {\left[G_{d H D 2}\right]_{p r}^{(1)}=-\frac{2}{M_{1}^{2}}\left(Y_{1 U}^{\not B L}\right)_{p r},} \\
& {\left[G_{d H D 3}\right]_{p r}^{(1)}=\frac{2}{M_{1}^{2}}\left(Y_{1 U}^{\not B L}\right)_{p r}} \\
& {\left[G_{d H D 4}\right]_{p r}^{(1)}=\frac{2}{M_{1}^{2}}\left(Y_{1 U}^{\not B L}\right)_{p r} .}
\end{aligned}
$$

$\psi^{2} X H$ :

$$
\begin{aligned}
{\left[G_{e W}\right]_{p r}^{(1)}=} & -\frac{N_{c}}{8} g\left(\frac{1}{2}+L_{1}\right) \frac{\left(Y_{1 U}^{1 L}\right)_{p r}}{M_{1}^{2}}, \\
{\left[G_{e B}\right]_{p r}^{(1)}=} & \frac{N_{c}}{4} g^{\prime}\left[\left(Y_{q}+Y_{u}\right) L_{1}+\frac{1}{2} Y_{q}+\frac{3}{2} Y_{u}\right] \frac{\left(Y_{1 U}^{1 L}\right)_{p r}}{M_{1}^{2}} \\
{\left[G_{u G}\right]_{p r}^{(1)}=} & g_{s}\left(1+L_{1}\right) \frac{\left(Y_{1 D}^{\not B L}\right)_{p r}}{M_{1}^{2}}, \\
{\left[G_{u W}\right]_{p r}^{(1)}=} & -\frac{1}{8} g\left[\left(\frac{1}{2}+L_{1}\right) \frac{\left(Y_{1 E}^{1 L}\right)_{p r}-4\left(Y_{1 D}^{\not B L}\right)_{p r}}{M_{1}^{2}}\right] \\
{\left[G_{u B}\right]_{p r}^{(1)}=} & \frac{1}{4} g^{\prime}\left[\left(Y_{\ell}+Y_{e}\right) L_{1}+\frac{1}{2} Y_{\ell}+\frac{3}{2} Y_{e}\right] \frac{\left(Y_{1 E}^{1 L}\right)_{p r}}{M_{1}^{2}} \\
& -g^{\prime}\left[\left(Y_{q}+Y_{d}\right) L_{1}+\frac{1}{2} Y_{q}+\frac{3}{2} Y_{d}\right] \frac{\left(Y_{1 D}^{\not B L}\right)_{p r}}{M_{1}^{2}} \\
{\left[G_{d G}\right]_{p r}^{(1)}=} & +g_{s}\left(1+L_{1}\right) \frac{\left(Y_{1 U}^{\not B L}\right)_{p r}}{M_{1}^{2}}, \\
{\left[G_{d W}\right]_{p r}^{(1)}=} & \frac{1}{2} g\left(\frac{1}{2}+L_{1}\right) \frac{\left(Y_{1 U}^{\not B L}\right)_{p r}}{M_{1}^{2}} \\
{\left[G_{d B}\right]_{p r}^{(1)}=} & -g^{\prime}\left[\left(Y_{u}+Y_{q}\right) L_{1}+\frac{1}{2} Y_{q}+\frac{3}{2} Y_{u}\right] \frac{\left(Y_{1 U}^{\not B L}\right)_{p r}}{M_{1}^{2}}
\end{aligned}
$$




\section{$\psi^{2} D H^{2}$ :}

$$
\begin{aligned}
& {\left[G_{H \ell}^{(1)}\right]_{p r}^{(1)}=-\frac{N_{c}}{4}\left[\left(1+L_{1}\right) \frac{\left(Y_{2 D}^{1 L}\right)_{p r}-\left(Y_{2 U}^{1 L}\right)_{p r}}{M_{1}^{2}}+\left(1+L_{2}\right) \frac{2\left(\tilde{Y}_{2 D}\right)_{p r}}{\tilde{M}_{2}^{2}}\right]} \\
& -\frac{N_{c}}{2}\left|A_{\tilde{2} 1}\right|^{2}\left[\frac{1}{\left(\Delta_{12}^{2}\right)}+\frac{M_{1}^{2}+\tilde{M}_{2}^{2}}{2\left(\Delta_{12}^{2}\right)^{2}} \log \frac{\tilde{M}_{2}^{2}}{M_{1}^{2}}\right] \frac{\left(\Lambda_{\ell}\right)_{p r}+\left(\tilde{\Lambda}_{\ell}\right)_{p r}}{\Delta_{12}^{2}}, \\
& {\left[G_{H \ell}^{\prime(1)}\right]_{p r}^{(1)}=-\frac{N_{c}}{8}\left[\frac{\left(Y_{2 U}^{1 L}\right)_{p r}+\left(Y_{2 D}^{1 L}\right)_{p r}+2 \lambda_{H 1}\left(\Lambda_{\ell}\right)_{p r}}{M_{1}^{2}}\right.} \\
& \left.+\frac{\left(2 \tilde{\lambda}_{H 2}-\lambda_{\tilde{2} \tilde{2}}\right)\left(\tilde{\Lambda}_{\ell}\right)_{p r}+\left(\tilde{Y}_{2 D}\right)_{p r}}{\tilde{M}_{2}^{2}}\right] \\
& -\frac{1}{4} \frac{\left|A_{\tilde{2} 1}\right|^{2}}{\Delta_{12}^{2}}\left[\frac{\log \tilde{M}_{2}^{2} / M_{1}^{2}}{\Delta_{12}^{2}}\left(\left(\Lambda_{\ell}\right)_{p r}+\left(\tilde{\Lambda}_{\ell}\right)_{p r}\right)+\left(\frac{\left(\Lambda_{\ell}\right)_{p r}}{M_{1}^{2}}+\frac{\left(\tilde{\Lambda}_{\ell}\right)_{p r}}{\tilde{M}_{2}^{2}}\right)\right], \\
& {\left[G_{H \ell}^{(3)}\right]_{p r}^{(1)}=\frac{N_{c}}{4}\left[\left(1+L_{1}\right) \frac{\left(Y_{2 D}^{1 L}\right)_{p r}+\left(Y_{2 U}^{1 L}\right)_{p r}}{M_{1}^{2}}\right] \text {, }} \\
& {\left[G_{H \ell}^{(3)}\right]_{p r}^{(1)}=\frac{N_{c}}{8}\left[\frac{\left(Y_{2 D}^{1 L}\right)_{p r}-\left(Y_{2 U}^{1 L}\right)_{p r}}{M_{1}^{2}}\right] \text {, }} \\
& {\left[G_{H e}\right]_{p r}^{(1)}=-\frac{N_{c}}{2}\left(1+L_{1}\right) \frac{\left(Y_{2 U}^{1 R}\right)_{p r}}{M_{1}^{2}}-\frac{N_{c}}{2}\left|A_{\tilde{2} 1}\right|^{2}\left[\frac{1}{\left(\Delta_{12}^{2}\right)}+\frac{M_{1}^{2}+\tilde{M}_{2}^{2}}{2\left(\Delta_{12}^{2}\right)^{2}} \log \frac{\tilde{M}_{2}^{2}}{M_{1}^{2}}\right] \frac{\left(\Lambda_{e}\right)_{p r}}{\Delta_{12}^{2}},} \\
& {\left[G_{H e}^{\prime}\right]_{p r}^{(1)}=-\frac{N_{c}}{4} \frac{\left(Y_{2 U}^{1 R}\right)_{p r}+\lambda_{H 1}\left(\Lambda_{e}\right)_{p r}}{M_{1}^{2}}-\frac{1}{4}\left|A_{\tilde{2} 1}\right|^{2}\left[\frac{1}{M_{1}^{2}}+\frac{\log \tilde{M}_{2}^{2} / M_{1}^{2}}{\Delta_{12}^{2}}\right] \frac{\left(\Lambda_{e}\right)_{p r}}{\Delta_{12}^{2}},} \\
& {\left[G_{H q}^{(1)}\right]_{p r}^{(1)}=-\frac{1}{4}\left[\left(1+L_{1}\right) \frac{\left(Y_{2 E}^{1 L}\right)_{p r}+8\left(Y_{2 D}^{\not B L}\right)_{p r}-8\left(Y_{2 U}^{\not B L}\right)_{p r}}{M_{1}^{2}}\right]} \\
& -\frac{1}{2}\left|A_{\tilde{2} 1}\right|^{2}\left[\frac{1}{\left(\Delta_{12}^{2}\right)}+\frac{M_{1}^{2}+\tilde{M}_{2}^{2}}{2\left(\Delta_{12}^{2}\right)^{2}} \log \frac{\tilde{M}_{2}^{2}}{M_{1}^{2}}\right] \frac{\left(\Lambda_{q}\right)_{p r}-8\left(\Lambda_{q}^{\not B}\right)_{p r}}{\Delta_{12}^{2}}, \\
& {\left[G_{H q}^{\prime(1)}\right]_{p r}^{(1)}=-\frac{1}{8}\left[\frac{\left(Y_{2 E}^{1 L}\right)_{p r}+2 \lambda_{H 1}\left(\Lambda_{q}\right)_{p r}+16 \lambda_{H 1}\left(\Lambda_{q}^{\not B}\right)_{p r}+8\left(Y_{2 D}^{\not B L}\right)_{p r}-8\left(Y_{2 U}^{\not B L}\right)_{p r}}{M_{1}^{2}}\right]} \\
& -\frac{1}{4}\left|A_{\tilde{2} 1}\right|^{2}\left[\frac{1}{M_{1}^{2}}+\frac{\log \tilde{M}_{2}^{2} / M_{1}^{2}}{\Delta_{12}^{2}}\right] \frac{\left(\Lambda_{q}\right)_{p r}+8\left(\Lambda_{q}^{\not B}\right)_{p r}}{\Delta_{12}^{2}}, \\
& {\left[G_{H q}^{(3)}\right]_{p r}^{(1)}=\frac{1}{4}\left[\left(1+L_{1}\right) \frac{\left(Y_{2 E}^{1 L}\right)_{p r}+8\left(Y_{2 D}^{\not B L}\right)_{p r}-8\left(Y_{2 U}^{\not B L}\right)_{p r}}{M_{1}^{2}}\right]} \\
& {\left[G_{H q}^{\prime(3)}\right]_{p r}^{(1)}=\frac{1}{8}\left[\frac{\left(Y_{2 E}^{1 L}\right)_{p r}+8\left(Y_{2 D}^{\not B L}\right)_{p r}-8\left(Y_{2 U}^{\not B L}\right)_{p r}}{M_{1}^{2}}\right],} \\
& {\left[G_{H u}\right]_{p r}^{(1)}=\frac{1}{2}\left(1+L_{1}\right) \frac{\left(Y_{2 E}^{1 R}\right)_{p r}+2\left(Y_{2 D}^{\not B R}\right)_{p r}}{M_{1}^{2}}} \\
& -\frac{1}{2}\left|A_{\tilde{2} 1}\right|^{2}\left[\frac{1}{\left(\Delta_{12}^{2}\right)}+\frac{M_{1}^{2}+\tilde{M}_{2}^{2}}{2\left(\Delta_{12}^{2}\right)^{2}} \log \frac{\tilde{M}_{2}^{2}}{M_{1}^{2}}\right] \frac{\left(\Lambda_{u}\right)_{p r}-2\left(\Lambda_{u}^{\not B}\right)_{p r}}{\Delta_{12}^{2}},
\end{aligned}
$$




$$
\begin{aligned}
& {\left[G_{H u}^{\prime}\right]_{p r}^{(1)}=-\frac{1}{4}\left[\frac{\left(Y_{2 E}^{1 R}\right)_{p r}+\lambda_{H 1}\left(\Lambda_{u}\right)_{p r}+2 \lambda_{H 1}\left(\Lambda_{u}^{\not B}\right)_{p r}+2\left(Y_{2 D}^{\not B R}\right)_{p r}}{M_{1}^{2}}\right]} \\
& -\frac{1}{4}\left|A_{\tilde{2} 1}\right|^{2}\left[\frac{1}{M_{1}^{2}}+\frac{\log \tilde{M}_{2}^{2} / M_{1}^{2}}{\Delta_{12}^{2}}\right] \frac{\left(\Lambda_{u}\right)_{p r}+2\left(\Lambda_{u}^{\not B}\right)_{p r}}{\Delta_{12}^{2}}, \\
& {\left[G_{H d}\right]_{p r}^{(1)}=\frac{1}{2}\left(1+L_{2}\right) \frac{\left(\tilde{Y}_{2 E}\right)_{p r}}{\tilde{M}_{2}^{2}}-\left(1+L_{1}\right) \frac{\left(Y_{2 U}^{\not B R}\right)_{p r}}{M_{1}^{2}}} \\
& -\frac{1}{2}\left|A_{\tilde{2} 1}\right|^{2}\left[\frac{1}{\left(\Delta_{12}^{2}\right)}+\frac{M_{1}^{2}+\tilde{M}_{2}^{2}}{2\left(\Delta_{12}^{2}\right)^{2}} \log \frac{\tilde{M}_{2}^{2}}{M_{1}^{2}}\right] \frac{\left(\tilde{\Lambda}_{d}\right)_{p r}-2\left(\Lambda_{d}^{\not B}\right)_{p r}}{\Delta_{12}^{2}}, \\
& {\left[G_{H d}^{\prime}\right]_{p r}^{(1)}=-\frac{1}{2} \frac{\left(Y_{2 U}^{\not B R}\right)_{p r}+\lambda_{H 1}\left(\Lambda_{d}^{\not B}\right)_{p r}}{M_{1}^{2}}-\frac{1}{4} \frac{\left(\tilde{Y}_{2 E}\right)_{p r}+\left(2 \lambda_{H 2}-\lambda_{\tilde{2} \tilde{2}}\right)\left(\tilde{\Lambda}_{d}\right)_{p r}}{\tilde{M}_{2}^{2}}} \\
& -\frac{1}{4} \frac{\left|A_{\tilde{2} 1}\right|^{2}}{\Delta_{12}^{2}}\left[\frac{\log \tilde{M}_{2}^{2} / M_{1}^{2}}{\Delta_{12}^{2}}\left(\left(\tilde{\Lambda}_{d}\right)_{p r}+2\left(\Lambda_{d}^{\not B}\right)_{p r}\right)+\left(\frac{\left(\tilde{\Lambda}_{d}\right)_{p r}}{M_{1}^{2}}+\frac{2\left(\Lambda_{d}^{\not B}\right)_{p r}}{\tilde{M}_{2}^{2}}\right)\right] \text {, } \\
& {\left[G_{H(d, u, e)}^{\prime \prime}\right]_{p r}^{(1)}=\left[G_{H \ell}^{\prime \prime(1,3)}\right]_{p r}^{(1)}=\left[G_{H q}^{\prime \prime(1,3)}\right]_{p r}^{(1)}=0 .} \\
& \psi^{2} H^{3} \text { : } \\
& \begin{aligned}
{\left[G_{e H}\right]_{p r}^{(1)}=} & N_{c} \frac{\left(1+L_{1}\right)\left(Y_{3 U}^{1 L}\right)_{p r}-\lambda_{H 1}\left(Y_{1 U}^{1 L}\right)_{p r}}{M_{1}^{2}} \\
& -N_{c}\left|A_{\tilde{2} 1}\right|^{2}\left(\frac{1}{M_{1}^{2}}+\frac{\log \tilde{M}_{2}^{2} / M_{1}^{2}}{\Delta_{12}^{2}}\right) \frac{\left(Y_{1 U}^{1 L}\right)_{p r}}{\Delta_{12}^{2}} \\
{\left[G_{u H}\right]_{p r}^{(1)}=} & \frac{\left(1+L_{1}\right)\left[\left(Y_{3 E}^{1 L}\right)_{p r}-4\left(Y_{3 D}^{\not B L}\right)_{p r}\right]-\lambda_{H 1}\left[\left(Y_{1 E}^{1 L}\right)_{p r}-4\left(Y_{1 D}^{\not B L}\right)_{p r}\right]}{M_{1}^{2}} \\
& -\left|A_{\tilde{2} 1}\right|^{2}\left(\frac{1}{M_{1}^{2}}+\frac{\log \tilde{M}_{2}^{2} / M_{1}^{2}}{\Delta_{12}^{2}}\right) \frac{\left(Y_{1 E}^{1 L}\right)_{p r}+4\left(Y_{1 D}^{\not B L}\right)_{p r}}{\Delta_{12}^{2}} \\
{\left[G_{d H}\right]_{p r}^{(1)}=} & -\frac{4\left(1+L_{1}\right)\left(Y_{3 U}^{\not B L}\right)_{p r}-4 \lambda_{H 1}\left(Y_{1 U}^{\not B L}\right)_{p r}}{M_{1}^{2}} \\
& -4\left|A_{\tilde{2} 1}\right|^{2}\left(\frac{1}{M_{1}^{2}}+\frac{\log \tilde{M}_{2}^{2} / M_{1}^{2}}{\Delta_{12}^{2}}\right) \frac{\left(Y_{1 U}^{\not B L}\right)_{p r}}{\Delta_{12}^{2}}
\end{aligned}
\end{aligned}
$$

\subsubsection{Four fermion operators}

\section{Four quarks:}

$$
\begin{aligned}
{\left[G_{q q}^{(1)}\right]_{p r s t}^{(1)}=} & -\frac{1}{16} \frac{\left(\Lambda_{q}\right)_{p t}\left(\Lambda_{q}\right)_{s r}}{M_{1}^{2}} \\
& -\frac{4\left(\Lambda_{q}^{\not B}\right)_{p r}\left(\Lambda_{q}^{\not B}\right)_{s t}+2\left(\Lambda_{q}^{\not B}\right)_{p t}\left(\Lambda_{q}^{\not B}\right)_{s r}+2\left(\Lambda_{q}^{\not B}\right)_{p r}\left(\Lambda_{q}\right)_{s t}-2\left(\Lambda_{q}^{\not B}\right)_{p t}\left(\Lambda_{q}\right)_{s r}}{M_{1}^{2}} \\
& +\left[\left(1+N_{c}\right)\left(1+L_{1}\right) c_{1}+\frac{2 \tilde{M}_{2}^{2}}{M_{1}^{2}}\left(1+L_{2}\right)\left(N_{c} c_{1 \tilde{2}}^{(1)}+c_{1 \tilde{2}}^{(2)}\right)\right. \\
& \left.+8\left|A^{\prime}\right|^{2} \frac{1}{M_{1}^{2}} L_{2}\right] \frac{\left(\lambda_{p s}^{\not B L}\right)\left(\lambda_{r t}^{\not B L}\right)^{*}}{2 M_{1}^{2}}
\end{aligned}
$$




$$
\begin{aligned}
& -\frac{2\left(\lambda_{p s}^{\not B L}\right)\left(\lambda_{r t}^{\not B L}\right)^{*}}{M_{1}^{2}}\left[\left(g^{\prime 2} Y_{q}^{2}-\frac{g_{s}^{2}}{12}-\frac{3 g^{2}}{4}\right)\left(\frac{1}{2}+a_{\mathrm{ev}}\right)\right. \\
& \left.+\left(\frac{g_{s}^{2}}{6}+\frac{3 g^{2}}{4}\right)\left(1+L_{1}\right)\right], \\
& {\left[G_{q q}^{(3)}\right]_{p r s t}^{(1)}=-\frac{1}{16} \frac{\left(\Lambda_{q}\right)_{p t}\left(\Lambda_{q}\right)_{s r}}{M_{1}^{2}}-2 \frac{\left(\Lambda_{q}^{\not B}\right)_{p t}\left(\Lambda_{q}^{\not B}\right)_{s r}}{M_{1}^{2}}} \\
& -\left[\left(1+N_{c}\right)\left(1+L_{1}\right) c_{1}+\frac{2 \tilde{M}_{2}^{2}}{M_{1}^{2}}\left(1+L_{2}\right)\left(N_{c} c_{1 \tilde{2}}^{(1)}+c_{1 \tilde{2}}^{(2)}\right)\right. \\
& \left.+8\left|A^{\prime}\right|^{2} \frac{1}{M_{1}^{2}} L_{2}\right] \frac{\left(\lambda_{p s}^{\not B L}\right)\left(\lambda_{r t}^{\not B L}\right)^{*}}{2 M_{1}^{2}} \\
& +\frac{2\left(\lambda_{p s}^{\not B L}\right)\left(\lambda_{r t}^{\not B L}\right)^{*}}{M_{1}^{2}}\left[\left(g^{\prime 2} Y_{q}^{2}-\frac{g_{s}^{2}}{12}-\frac{g^{2}}{4}\right)\left(\frac{1}{2}+a_{\mathrm{ev}}\right)\right. \\
& \left.+\left(\frac{g_{s}^{2}}{6}+\frac{g^{2}}{4}\right)\left(1+L_{1}\right)\right], \\
& {\left[G_{u u}\right]_{p r s t}^{(1)}=-\frac{1}{8 M_{1}^{2}}\left[\left(\Lambda_{u}\right)_{p t}\left(\Lambda_{u}\right)_{s r}+2\left(\Lambda_{u}^{\not B}\right)_{p r}\left(\Lambda_{u}^{\not B}\right)_{s t}+2\left(\Lambda_{u}^{\not B}\right)_{s r}\left(\Lambda_{u}^{\not B}\right)_{p t}\right.} \\
& \left.+4\left(\Lambda_{u}\right)_{p r}\left(\Lambda_{u}^{\not B}\right)_{s t}-4\left(\Lambda_{u}\right)_{p t}\left(\Lambda_{u}^{\not B}\right)_{s r}\right], \\
& {\left[G_{d d}\right]_{p r s t}^{(1)}=\frac{1}{4} \frac{\left(\tilde{\Lambda}_{d}\right)_{p t}\left(\tilde{\Lambda}_{d}\right)_{s r}-2\left(\Lambda_{d}^{\not B}\right)_{p r}\left(\Lambda_{d}^{\not B}\right)_{s t}-2\left(\Lambda_{d}^{\not B}\right)_{s r}\left(\Lambda_{d}^{\not B}\right)_{p t}}{\tilde{M}_{2}^{2}},} \\
& {\left[G_{u d}^{(1)}\right]_{p r s t}^{(1)}=\frac{\left(\Lambda_{u}\right)_{p r}\left(\Lambda_{d}^{\not B}\right)_{s t}}{3 M_{1}^{2}}-\frac{3}{4} \frac{\left(\Lambda_{u}^{\not B}\right)_{p r}\left(\Lambda_{d}^{\not B}\right)_{s t}}{M_{1}^{1}}} \\
& +\left[\left(1+N_{c}\right)\left(1+L_{1}\right) c_{1}+\frac{2 \tilde{M}_{2}^{2}}{M_{1}^{2}}\left(1+L_{2}\right)\left(N_{c} c_{1 \tilde{2}}^{(1)}+c_{1 \tilde{2}}^{(2)}\right)\right. \\
& \left.+8\left|A^{\prime}\right|^{2} \frac{1}{M_{1}^{2}} L_{2}\right] \frac{\left(\lambda_{p s}^{\not B R}\right)^{T}\left(\lambda_{r t}^{\not B R}\right)^{\dagger}}{3 M_{1}^{2}} \\
& +g^{\prime 2} \frac{\left(\lambda_{p s}^{\not B R}\right)^{T}\left(\lambda_{t r}^{\not B R}\right)^{*}}{3 M_{1}^{2}}\left[\left(Y_{u}-Y_{d}\right)^{2}\left(\frac{1}{2}+a_{\mathrm{ev}}\right)-\left(Y_{u}+Y_{d}+Y_{S_{1}}\right)^{2}\left(1+L_{1}\right)\right] \\
& +g_{s}^{2}\left[\frac{4}{3}\left(\frac{1}{2}+a_{e v}\right)+\frac{5}{9}\left(1+L_{1}\right)\right] \frac{\left(\lambda_{t r}^{\not B R}\right)^{*}\left(\lambda_{s p}^{\not B R}\right)}{M_{1}^{2}}, \\
& {\left[G_{u d}^{(8)}\right]_{p r s t}^{(1)}=-\frac{\left(\Lambda_{u}\right)_{p r}\left(\Lambda_{d}^{\not B}\right)_{s t}}{M_{1}^{2}}-\frac{\left(\Lambda_{u}^{\not B}\right)_{p r}\left(\Lambda_{d}^{\not B}\right)_{s t}}{M_{1}^{1}}} \\
& -\left[\left(1+N_{c}\right)\left(1+L_{1}\right) c_{1}+\frac{2 \tilde{M}_{2}^{2}}{M_{1}^{2}}\left(1+L_{2}\right)\left(N_{c} c_{1 \tilde{2}}^{(1)}+c_{1 \tilde{2}}^{(2)}\right)\right. \\
& \left.+8\left|A^{\prime}\right|^{2} \frac{1}{M_{1}^{2}} L_{2}\right] \frac{\left(\lambda_{p s}^{\not B R}\right)^{T}\left(\lambda_{r t}^{\not B R}\right)^{\dagger}}{M_{1}^{2}} \\
& -g^{\prime 2} \frac{\left(\lambda_{p s}^{\not B R}\right)^{T}\left(\lambda_{t r}^{\not B R}\right)^{*}}{M_{1}^{2}}\left[\left(Y_{u}-Y_{d}\right)^{2}\left(\frac{1}{2}+a_{\mathrm{ev}}\right)-\left(Y_{u}+Y_{d}+Y_{S_{1}}\right)^{2}\left(1+L_{1}\right)\right] \\
& +g_{s}^{2}\left[\frac{7}{6}\left(\frac{1}{2}+a_{e v}\right)+\frac{13}{6}\left(1+L_{1}\right)\right] \frac{\left(\lambda_{t r}^{\not B R}\right)^{*}\left(\lambda_{s p}^{\not B R}\right)}{M_{1}^{2}} ;
\end{aligned}
$$




$$
\begin{aligned}
& {\left[G_{q u}^{(1)}\right]_{p r s t}^{(1)}=-\frac{1}{12} \frac{\left(\Lambda_{q}\right)_{p r}\left(\Lambda_{u}\right)_{s t}}{M_{1}^{2}}-\frac{3\left(\Lambda_{q}^{\not B}\right)_{p r}\left(\Lambda_{u}^{\not B}\right)_{s t}}{M_{1}^{2}}+\frac{\left(\Lambda_{q}\right)_{p r}\left(\Lambda_{u}^{\not B}\right)_{s t}-4\left(\Lambda_{q}^{\not B}\right)_{p r}\left(\Lambda_{u}\right)_{s t}}{3 M_{1}^{2}}} \\
& +\left(\frac{3}{2}+L_{1}\right) \frac{\left(y_{D} \lambda^{\not B R}+2 \lambda^{\not B L} y_{U}^{*}\right)_{p s}\left(y_{D}^{*}\left(\lambda^{\not B R}\right)^{*}+2\left(\lambda^{\not B L}\right)^{*} y_{U}\right)_{r t}}{6 M_{1}^{2}}, \\
& {\left[G_{q u}^{(8)}\right]_{p r s t}^{(1)}=-\frac{1}{2} \frac{\left(\Lambda_{q}\right)_{p r}\left(\Lambda_{u}\right)_{s t}}{M_{1}^{2}}-\frac{4\left(\Lambda_{q}^{\not B}\right)_{p r}\left(\Lambda_{u}^{\not B}\right)_{s t}}{M_{1}^{2}}-\frac{\left(\Lambda_{q}\right)_{p r}\left(\Lambda_{u}^{\not B}\right)_{s t}-4\left(\Lambda_{q}^{\not B}\right)_{p r}\left(\Lambda_{u}\right)_{s t}}{M_{1}^{2}}} \\
& -\left(\frac{3}{2}+L_{1}\right) \frac{\left(y_{D} \lambda^{\not B R}+2 \lambda^{\not B L} y_{U}^{*}\right)_{p s}\left(y_{D}^{*}\left(\lambda^{\not B R}\right)^{*}+2\left(\lambda^{\not B L}\right)^{*} y_{U}\right)_{r t}}{2 M_{1}^{2}}, \\
& {\left[G_{q d}^{(1)}\right]_{p r s t}^{(1)}=\frac{\left(\Lambda_{q}\right)_{p r}\left(\Lambda_{d}^{\not B}\right)_{s t}}{3 M_{1}^{2}}-\frac{3\left(\Lambda_{q}^{\not B}\right)_{p r}\left(\Lambda_{d}^{\not B}\right)_{s t}}{M_{1}^{2}}-\frac{1}{8} \frac{\left(\left(\lambda^{1 \mathrm{~L}}\right)^{*} \tilde{\lambda}^{T}\right)_{p s}\left(\tilde{\lambda}^{*}\left(\lambda^{1 \mathrm{~L}}\right)^{T}\right)_{t r}}{M_{1}^{2}-\tilde{M}_{2}^{2}} \log \frac{M_{1}^{2}}{\tilde{M}_{2}^{2}}} \\
& -\left(\frac{3}{2}+L_{1}\right) \frac{\left(y_{U}\left(\lambda^{\not B R}\right)^{T}+2 \lambda^{\not B L} y_{D}^{*}\right)_{p s}\left(y_{U}^{*}\left(\lambda^{\not B R}\right)^{\dagger}+2\left(\lambda^{\not B L}\right)^{*} y_{D}\right)_{r t}}{6 M_{1}^{2}} \\
& {\left[G_{q d}^{(8)}\right]_{p r s t}^{(1)}=-\frac{\left(\Lambda_{q}\right)_{p r}\left(\Lambda_{d}^{\not B}\right)_{s t}}{M_{1}^{2}}-\frac{4\left(\Lambda_{q}^{\not B}\right)_{p r}\left(\Lambda_{d}^{\not B}\right)_{s t}}{M_{1}^{2}}} \\
& +\left(\frac{3}{2}+L_{1}\right) \frac{\left(y_{U}\left(\lambda^{\not B R}\right)^{T}+2 \lambda^{\not B L} y_{D}^{*}\right)_{p s}\left(y_{U}^{*}\left(\lambda^{\not B R}\right)^{\dagger}+2\left(\lambda^{\not B L}\right)^{*} y_{D}\right)_{r t}}{2 M_{1}^{2}}, \\
& {\left[G_{q u q d}^{(1)}\right]_{p r s t}^{(1)}=\frac{4}{3}\left[\left(1+N_{c}\right)\left(1+L_{1}\right) c_{1}+\frac{2 \tilde{M}_{2}^{2}}{M_{1}^{2}}\left(1+L_{2}\right)\left(N_{c} c_{1 \tilde{2}}^{(1)}+c_{1 \tilde{2}}^{(2)}\right)\right.} \\
& \left.+8\left|A^{\prime}\right|^{2} \frac{1}{M_{1}^{2}} L_{2}\right] \frac{\left(\lambda_{p r}^{\not B L}\right)\left(\lambda_{s t}^{\not B R}\right)^{\dagger}}{M_{1}^{2}} \\
& -\frac{4}{3} g^{\prime 2} \frac{\left(\lambda_{p s}^{\not B L}\right)\left(\lambda_{t r}^{\not B R}\right)^{*}}{M_{1}^{2}}\left(1+L_{1}\right)\left[Y_{q}\left(Y_{S_{1}}+2 Y_{q}\right)+Y_{d}\left(Y_{d}-Y_{S_{1}}\right)\right] \\
& +\frac{44}{9} g_{s}^{2} \frac{\left(\lambda_{t r}^{\not B R}\right)^{*}\left(\lambda_{s p}^{\not B L}\right)}{M_{1}^{2}}\left(\frac{8}{11}+L_{1}\right) \\
& {\left[G_{q u q d}^{(8)}\right]_{p r s t}^{(1)}=-4\left[\left(1+N_{c}\right)\left(1+L_{1}\right) c_{1}+\frac{2 \tilde{M}_{2}^{2}}{M_{1}^{2}}\left(1+L_{2}\right)\left(N_{c} c_{1 \tilde{2}}^{(1)}+c_{1 \tilde{2}}^{(2)}\right)\right.} \\
& \left.+8\left|A^{\prime}\right|^{2} \frac{1}{M_{1}^{2}} L_{2}\right] \frac{\left(\lambda_{p r}^{\not B L}\right)\left(\lambda_{s t}^{\not B R}\right)^{\dagger}}{M_{1}^{2}} \\
& +4 g^{\prime 2} \frac{\left(\lambda_{p s}^{\not B L}\right)\left(\lambda_{t r}^{\not B R}\right)^{*}}{M_{1}^{2}}\left(1+L_{1}\right)\left[Y_{q}\left(Y_{S_{1}}+2 Y_{q}\right)+Y_{d}\left(Y_{d}-Y_{S_{1}}\right)\right] \\
& +\frac{2}{3} g_{s}^{2} \frac{\left(\lambda_{t r}^{\not B R}\right)^{*}\left(\lambda_{s p}^{\not B L}\right)}{M_{1}^{2}}
\end{aligned}
$$

\section{Four leptons:}

$$
\begin{aligned}
{\left[G_{\ell \ell}^{(1)}\right]_{p r s t}^{(1)} } & =-\frac{N_{c}}{8}\left[\frac{\left(\Lambda_{\ell}\right)_{p r}\left(\Lambda_{\ell}\right)_{s t}}{M_{1}^{2}}+\frac{\left(\tilde{\Lambda}_{\ell}\right)_{p t}\left(\tilde{\Lambda}_{\ell}\right)_{s r}}{\tilde{M}_{2}^{2}}\right] \\
{\left[G_{e e}\right]_{p r s t}^{(1)} } & =-\frac{N_{c}}{8} \frac{\left(\Lambda_{e}\right)_{p r}\left(\Lambda_{e}\right)_{s t}}{M_{1}^{2}} \\
{\left[G_{\ell e}\right]_{p r s t}^{(1)} } & =-\frac{N_{c}}{4} \frac{\left(\Lambda_{\ell}\right)_{p r}\left(\Lambda_{e}\right)_{s t}}{M_{1}^{2}} .
\end{aligned}
$$




\section{Semileptonic:}

$$
\begin{aligned}
& {\left[G_{\ell q}^{(1)}\right]_{p r s t}^{(1)}=-\frac{1}{4} \frac{\left(\Lambda_{\ell}\right)_{p r}\left(\Lambda_{q}\right)_{s t}}{M_{1}^{2}}-\frac{4\left(\Lambda_{\ell}\right)_{p r}\left(\Lambda_{q}^{\not B}\right)_{s t}}{M_{1}^{2}}+\frac{4\left(\lambda^{\not B L} \lambda^{1 \mathrm{~L}}\right)_{t p}^{*}\left(\left(\lambda^{1 \mathrm{~L}}\right)^{T} \lambda^{\not B L}\right)_{r s}}{M_{1}^{2}}} \\
& +\frac{1}{4}\left(\frac{3}{2}+L_{2}\right) \frac{\left(\tilde{\lambda}^{\dagger} y_{D}^{\dagger}\right)_{p t}\left(y_{D} \tilde{\lambda}\right)_{s r}}{\tilde{M}_{2}^{2}} \\
& +\left[\frac{1+N_{c}}{4}\left(1+L_{1}\right) c_{1}+\frac{\tilde{M}_{2}^{2}}{2 M_{1}^{2}}\left(1+L_{2}\right)\left(N_{c} c_{1 \tilde{2}}^{(1)}+c_{1 \tilde{2}}^{(2)}\right)\right. \\
& \left.+8\left|A^{\prime}\right|^{2} \frac{1}{M_{1}^{2}} L_{2}\right] \frac{\left(\lambda_{p s}^{1 \mathrm{~L}}\right)^{\dagger}\left(\lambda_{t r}^{1 \mathrm{~L}}\right)}{M_{1}^{2}} \\
& +\frac{1}{4}\left(\frac{1}{2}+a_{\mathrm{ev}}\right)\left[g_{s}^{2} C_{F}^{\mathrm{SU}(3)}+g^{\prime 2}\left(Y_{q}-Y_{\ell}\right)^{2}+3 g^{2}\right] \frac{\left(\lambda_{p s}^{1 \mathrm{~L}}\right)^{\dagger} \lambda_{t r}^{1 \mathrm{~L}}}{M_{1}^{2}}, \\
& {\left[G_{\ell q}^{(3)}\right]_{p r s t}^{(1)}=\frac{4\left(\lambda^{\not B L} \lambda^{1 \mathrm{~L}}\right)_{t p}^{*}\left(\left(\lambda^{1 \mathrm{~L}}\right)^{T} \lambda^{\not B L}\right)_{r s}}{M_{1}^{2}}} \\
& -\left[\frac{1+N_{c}}{4}\left(1+L_{1}\right) c_{1}+\frac{\tilde{M}_{2}^{2}}{2 M_{1}^{2}}\left(1+L_{2}\right)\left(N_{c} c_{1 \tilde{2}}^{(1)}+c_{1 \tilde{2}}^{(2)}\right)\right. \\
& \left.+8\left|A^{\prime}\right|^{2} \frac{1}{M_{1}^{2}} L_{2}\right] \frac{\left(\lambda_{p s}^{1 \mathrm{~L}}\right)^{\dagger}\left(\lambda_{t r}^{1 \mathrm{~L}}\right)}{M_{1}^{2}} \\
& -\frac{1}{4}\left(\frac{1}{2}+a_{\mathrm{ev}}\right)\left[g_{s}^{2} C_{F}^{\mathrm{SU}(3)}+g^{\prime 2}\left(Y_{q}-Y_{\ell}\right)^{2}-3 g^{2}\right] \frac{\left(\lambda_{p s}^{1 \mathrm{~L}}\right)^{\dagger} \lambda_{t r}^{1 \mathrm{~L}}}{M_{1}^{2}}, \\
& {\left[G_{e u}\right]_{p r s t}^{(1)}=\left[\frac{1+N_{c}}{2}\left(1+L_{1}\right) c_{1}+\frac{\tilde{M}_{2}^{2}}{M_{1}^{2}}\left(1+L_{2}\right)\left(N_{c} c_{1 \tilde{2}}^{(1)}+c_{1 \tilde{2}}^{(2)}\right)\right.} \\
& \left.+8\left|A^{\prime}\right|^{2} \frac{1}{M_{1}^{2}} L_{2}\right] \frac{\left(\lambda_{p s}^{1 \mathrm{R}}\right)^{\dagger}\left(\lambda_{t r}^{1 \mathrm{R}}\right)}{M_{1}^{2}} \\
& +\frac{1}{2}\left(\frac{1}{2}+a_{\mathrm{ev}}\right)\left[g_{s}^{2} C_{F}^{\mathrm{SU}(3)}+g^{\prime 2}\left(Y_{u}-Y_{e}\right)^{2}+3 g^{2}\right] \frac{\left(\lambda_{p s}^{1 \mathrm{R}}\right)^{\dagger} \lambda_{t r}^{1 \mathrm{R}}}{M_{1}^{2}}+\frac{\left(\Lambda_{e}\right)_{p r}\left(\Lambda_{u}^{\not B}\right)_{s t}}{M_{1}^{2}},
\end{aligned}
$$

$$
\left[G_{e d}\right]_{p r s t}^{(1)}=\frac{\left(\Lambda_{e}\right)_{p r}\left(\Lambda_{d}^{\not B}\right)_{s t}}{M_{1}^{2}}-\frac{2\left(\lambda^{\not B R} \lambda^{1 \mathrm{R}}\right)_{t p}^{*}\left(\lambda^{\not B R} \lambda^{1 \mathrm{R}}\right)_{s r}}{M_{1}^{2}}-\frac{1}{2}\left(\frac{3}{2}-L_{2}\right) \frac{\left(y_{E}^{\dagger} \tilde{\lambda}^{\dagger}\right)_{p t}\left(\tilde{\lambda} y_{E}\right)_{s r}}{\tilde{M}_{2}^{2}}
$$

$$
\begin{aligned}
{\left[G_{q e}\right]_{p r s t}^{(1)}=} & -\frac{1}{4} \frac{\left(\Lambda_{q}\right)_{p r}\left(\Lambda_{e}\right)_{s t}}{M_{1}^{2}}-\frac{4\left(\Lambda_{q}^{\not B}\right)_{p r}\left(\Lambda_{e}\right)_{s t}}{M_{1}^{2}} \\
& -\frac{1}{4}\left(\frac{3}{2}+L_{1}\right) \frac{\left(\left(\lambda^{1 \mathrm{~L}}\right)^{*} y_{E}^{*}-y_{U}\left(\lambda^{1 \mathrm{R}}\right)^{*}\right)_{p s}\left(\lambda^{1 \mathrm{~L}} y_{E}-y_{U}^{*} \lambda^{1 \mathrm{R}}\right)_{r t}}{M_{1}^{2}}
\end{aligned}
$$

$$
\begin{aligned}
{\left[G_{\ell u}\right]_{p r s t}^{(1)}=} & -\frac{1}{4} \frac{\left(\Lambda_{\ell}\right)_{p r}\left(\Lambda_{u}\right)_{s t}}{M_{1}^{2}}+\frac{\left(\Lambda_{\ell}\right)_{p r}\left(\Lambda_{u}^{\not B}\right)_{s t}}{M_{1}^{2}} \\
& -\frac{1}{4}\left(\frac{3}{2}+L_{1}\right) \frac{\left(\left(\lambda^{1 \mathrm{~L}}\right)^{\dagger} y_{U}^{*}-y_{E}\left(\lambda^{1 \mathrm{R}}\right)^{\dagger}\right)_{p s}\left(\left(\lambda^{1 \mathrm{~L}}\right)^{T} y_{U}-y_{E}^{*}\left(\lambda^{1 \mathrm{R}}\right)^{T}\right)_{r t}}{M_{1}^{2}} \\
& +\frac{1}{2} \frac{\left(\tilde{\lambda}^{\dagger} \lambda^{\not B R}\right)_{p s}\left(\left(\lambda^{\not B R}\right)^{\dagger} \tilde{\lambda}\right)_{t r}}{M_{1}^{2}-\tilde{M}_{2}^{2}} \log \frac{M_{1}^{2}}{\tilde{M}_{2}^{2}}
\end{aligned}
$$




$$
\begin{aligned}
& {\left[G_{\ell d}\right]_{p r s t}^{(1)}=\frac{\left(\Lambda_{\ell}\right)_{p r}\left(\Lambda_{d}^{\not B}\right)_{s t}}{M_{1}^{2}}+\frac{1}{4} \frac{\left(\tilde{\Lambda}_{\ell}\right)_{p r}\left(\tilde{\Lambda}_{d}\right)_{s t}}{M_{1}^{2}-\tilde{M}_{2}^{2}} \log \frac{M_{1}^{2}}{\tilde{M}_{2}^{2}}} \\
& -\frac{1}{4}\left(\frac{3}{2}+L_{1}\right) \frac{\left(\left(\lambda^{1 \mathrm{~L}}\right)^{\dagger} y_{D}^{*}\right)_{p s}\left(\left(\lambda^{1 \mathrm{~L}}\right)^{T} y_{D}\right)_{r t}}{M_{1}^{2}} \\
& -\left[\frac{M_{1}^{2}}{\tilde{M}_{2}^{2}}\left(1+L_{1}\right)\left(N_{c} c_{1 \tilde{2}}^{(1)}+c_{1 \tilde{2}}^{(2)}\right)\right. \\
& \left.+\left(\left(1+2 N_{c}\right) \tilde{c}_{2}+\left(2+N_{c}\right) c_{\tilde{2}}^{(8)}\right)\left(1+L_{2}\right)\right] \frac{\tilde{\lambda}_{p t}^{\dagger} \tilde{\lambda}_{s r}}{2 \tilde{M}_{2}^{2}} \\
& -\frac{1}{2}\left(\frac{1}{2}+a_{\mathrm{ev}}\right)\left[g_{2}^{2} C_{F}^{\mathrm{SU}(3)}+g^{2} C_{F}^{\mathrm{SU}(2)}+g^{\prime 2}\left(Y_{\ell}+Y_{d}\right)^{2}\right] \frac{\left(\tilde{\lambda}_{p t}^{*}\right)\left(\tilde{\lambda}_{s r}\right)}{\tilde{M}_{2}^{2}} \\
& -2\left|A^{\prime}\right|^{2} \frac{\left(\tilde{\lambda}_{p t}\right)^{\dagger}\left(\tilde{\lambda}_{s r}\right)}{\tilde{M}_{2}^{4}}\left(1+\frac{M_{1}^{2} L_{1}-\tilde{M}_{2}^{2} L_{2}}{\Delta_{12}^{2}}\right), \\
& {\left[G_{\text {ledq }}\right]_{p r s t}^{(1)}=-\frac{6\left(\lambda^{\not B L} \lambda^{1 \mathrm{~L}}\right)_{t p}^{*}\left(\lambda^{\not B R} \lambda^{1 \mathrm{R}}\right)_{s r}}{M_{1}^{2}}} \\
& -\frac{1}{2}\left(\frac{3}{2}+L_{1}\right) \frac{\left(\left(\lambda^{1 \mathrm{~L}}\right)^{\dagger} y_{D}^{*}\right)_{p s}\left(\lambda^{1 \mathrm{~L}} y_{E}-y_{U}^{*} \lambda^{1 \mathrm{R}}\right)_{t r}}{M_{1}^{2}} \\
& +\frac{1}{2}\left(\frac{3}{2}+L_{2}\right) \frac{\left(\tilde{\lambda}^{\dagger} y_{D}^{\dagger}\right)_{p t}\left(\tilde{\lambda} y_{E}\right)_{s r}}{\tilde{M}_{2}^{2}} \\
& {\left[G_{\text {lequ }}^{(1)}\right]_{p r s t}^{(1)}=\left[\frac{1+N_{c}}{2}\left(1+L_{1}\right) c_{1}+\frac{\tilde{M}_{2}^{2}}{M_{1}^{2}}\left(1+L_{2}\right)\left(N_{c} c_{1 \tilde{2}}^{(1)}+c_{1 \tilde{2}}^{(2)}\right)\right.} \\
& \left.+8\left|A^{\prime}\right|^{2} \frac{1}{M_{1}^{2}} L_{2}\right] \frac{\left(\lambda_{p t}^{1 \mathrm{~L}}\right)^{\dagger}\left(\lambda_{s r}^{1 \mathrm{R}}\right)}{M_{1}^{2}} \\
& -\frac{3}{2}\left(\frac{3}{2}+L_{1}\right)\left[g_{s}^{2} C_{F}^{\mathrm{SU}(3)}+g^{\prime 2}\left(Y_{q}-Y_{\ell}\right)\left(Y_{u}-Y_{e}\right)\right] \frac{\left(\lambda_{p s}^{1 \mathrm{~L}}\right)^{\dagger} \lambda_{t r}^{1 \mathrm{R}}}{M_{1}^{2}}, \\
& {\left[G_{\text {lequ }}^{(3)}\right]_{p r s t}^{(1)}=-\left[\frac{1+N_{c}}{8}\left(1+L_{1}\right) c_{1}+\frac{\tilde{M}_{2}^{2}}{4 M_{1}^{2}}\left(1+L_{2}\right)\left(N_{c} c_{1 \tilde{2}}^{(1)}+c_{1 \tilde{2}}^{(2)}\right)\right.} \\
& \left.+8\left|A^{\prime}\right|^{2} \frac{1}{M_{1}^{2}} L_{2}\right] \frac{\left(\lambda_{p t}^{1 \mathrm{~L}}\right)^{\dagger}\left(\lambda_{s r}^{1 \mathrm{R}}\right)}{M_{1}^{2}} \\
& -\frac{1}{8}\left(\frac{3}{2}+L_{1}\right)\left[g_{s}^{2} C_{F}^{\mathrm{SU}(3)}+g^{\prime 2}\left(Y_{q}-Y_{\ell}\right)\left(Y_{u}-Y_{e}\right)\right] \frac{\left(\lambda_{p s}^{1 \mathrm{~L}}\right)^{\dagger} \lambda_{t r}^{1 \mathrm{R}}}{M_{1}^{2}} .
\end{aligned}
$$

\section{B-violating:}

$$
\begin{aligned}
{\left[G_{d u q}\right]_{p r s t}^{(1)}=} & {\left[\left(1+N_{c}\right)\left(1+L_{1}\right) c_{1}+\frac{2 \tilde{M}_{2}^{2}}{M_{1}^{2}}\left(1+L_{2}\right)\left(N_{c} c_{1 \tilde{2}}^{(1)}+c_{1 \tilde{2}}^{(2)}\right)\right.} \\
& \left.+8\left|A^{\prime}\right|^{2} \frac{1}{M_{1}^{2}} L_{2}\right] \frac{\left(\lambda_{p r}^{\not B R}\right)^{*}\left(\lambda_{s t}^{1 L}\right)}{M_{1}^{2}} \\
& +\left(1+L_{1}\right)\left[g^{\prime 2}\left(Y_{d}\left(Y_{q}-Y_{S_{1}}\right)+Y_{u}\left(Y_{\ell}-Y_{S_{1}}\right)+Y_{d}\left(Y_{u}-Y_{S_{1}}\right)\right)\right] \frac{\left(\lambda_{p r}^{\not B R}\right)^{*}\left(\lambda_{s t}^{1 \mathrm{~L}}\right)}{M_{1}^{2}}
\end{aligned}
$$




$$
\begin{aligned}
& -\left(\frac{1}{2}+a_{e v}\right)\left(Y_{\ell}+Y_{u}\right)\left(Y_{u}+Y_{d}\right) \frac{\left(\lambda_{p r}^{\not B R}\right)^{*}\left(\lambda_{s t}^{1 \mathrm{~L}}\right)}{M_{1}^{2}} \\
& +g_{s}^{2}\left[\frac{4}{3}\left(\frac{1}{2}+a_{e v}\right)-\frac{13}{6}\left(1+L_{1}\right)\right] \frac{\left(\lambda_{p r}^{\not B R}\right)^{*}\left(\lambda_{s t}^{1 \mathrm{~L}}\right)}{M_{1}^{2}} \\
& +\frac{\left((\tilde{\lambda})^{*}\left(\lambda^{1 \mathrm{~L}}\right)^{T}\right)_{p s}\left(\left(\lambda^{\not B R}\right)^{\dagger} \tilde{\lambda}\right)_{r t}}{M_{1}^{2}-\tilde{M}_{2}^{2}} \log \frac{M_{1}^{2}}{\tilde{M}_{2}^{2}} \\
& +\frac{\left(\lambda^{1 \mathrm{~L}} y_{E}+y_{U}^{*} \lambda^{1 \mathrm{R}}\right)_{p t}\left(\left(\lambda^{\not B R}\right)^{\dagger} y_{D}^{\dagger}+2 y_{U}^{T}\left(\lambda^{\not B L}\right)^{*}\right)_{s r}}{2 M_{1}^{2}}\left(\frac{3}{2}+L_{1}\right), \\
& {\left[G_{q q u}\right]_{p r s t}^{(1)}=\left[\left(1+N_{c}\right)\left(1+L_{1}\right) c_{1}+\frac{2 \tilde{M}_{2}^{2}}{M_{1}^{2}}\left(1+L_{2}\right)\left(N_{c} c_{1 \tilde{2}}^{(1)}+c_{1 \tilde{2}}^{(2)}\right)\right.} \\
& \left.+8\left|A^{\prime}\right|^{2} \frac{1}{M_{1}^{2}} L_{2}\right] \frac{\left(\lambda_{p r}^{\not B L}\right)^{*}\left(\lambda_{s t}^{1 \mathrm{R}}\right)}{M_{1}^{2}} \\
& -\left(1+L_{1}\right)\left[4 g^{\prime 2} Y_{S_{1}}\left(2 Y_{q}+Y_{S_{1}}\right)\right] \frac{\left(\lambda_{p r}^{\not B L}\right)^{*}\left(\lambda_{s t}^{1 \mathrm{R}}\right)}{4 M_{1}^{2}} \\
& +2 g^{\prime 2}\left(\frac{1}{2}+a_{e v}\right) Y_{q}\left(Y_{e}-Y_{u}\right) \frac{\left(\lambda_{p r}^{\not B L}\right)^{*}\left(\lambda_{s t}^{1 \mathrm{R}}\right)}{M_{1}^{2}} \\
& +g_{s}^{2} \frac{\left(\lambda_{p r}^{\not B L}\right)^{*}\left(\lambda_{s t}^{1 \mathrm{R}}\right)}{M_{1}^{2}}\left[\frac{2}{3}\left(\frac{1}{2}+a_{e v}\right)-\frac{5}{2}\left(1+L_{1}\right)\right] \\
& +\frac{\left(2\left(\lambda^{\not B L}\right)^{*} y_{D}-y_{U}^{*}\left(\lambda^{\not B R}\right)^{\dagger}\right)_{s p}\left(y_{E}^{*}\left(\lambda^{1 \mathrm{R}}\right)^{T}-\left(\lambda^{1 \mathrm{~L}}\right)^{T} y_{U}\right)_{t r}}{2 M_{1}^{2}}\left(\frac{3}{2}+L_{1}\right) \\
& -\frac{\left(y_{D}^{T} \lambda^{1 \mathrm{~L}}\right)_{p t}\left(2\left(\lambda^{\not B L}\right)^{*} y_{U}+y_{D}^{*}\left(\lambda^{\not B R}\right)^{*}\right)_{s r}}{2 M_{1}^{2}}\left(\frac{3}{2}+L_{1}\right),
\end{aligned}
$$

$$
\begin{aligned}
{\left[G_{q q q}\right]_{p r s t}^{(1)}=-\left[\left(1+N_{c}\right)\left(1+L_{1}\right) c_{1}+\frac{2 \tilde{M}_{2}^{2}}{M_{1}^{2}}\left(1+L_{2}\right)\right.} & \left(N_{c} c_{1 \tilde{2}}^{(1)}+c_{1 \tilde{2}}^{(2)}\right) \\
& \left.+8\left|A^{\prime}\right|^{2} \frac{1}{M_{1}^{2}} L_{2}\right] \frac{2\left(\lambda_{p r}^{\not B L}\right)^{*}\left(\lambda_{s t}^{1 \mathrm{~L}}\right)}{M_{1}^{2}}
\end{aligned}
$$$$
+\left(1+L_{1}\right)\left[2 g^{\prime 2} Y_{S_{1}}\left(6 Y_{q}+Y_{\ell}\right)\right] \frac{\left(\lambda_{p r}^{\not B L}\right)^{*}\left(\lambda_{s t}^{1 \mathrm{~L}}\right)}{2 M_{1}^{2}}
$$$$
+g_{s}^{2}\left(\frac{3}{2}+L_{1}\right) \frac{\left(\lambda_{p s}^{\not B L}\right)^{*}\left(\lambda_{r t}^{1 \mathrm{~L}}\right)}{12 M_{1}^{2}}-\frac{5}{3} g_{s}^{2} \frac{\left(\lambda_{p r}^{\not B L}\right)^{*}\left(\lambda_{s t}^{1 \mathrm{~L}}\right)}{M_{1}^{2}}\left(-\frac{3}{5}+L_{1}\right)
$$

$$
\begin{aligned}
& +\frac{2 g^{2}}{M_{1}^{2}}\left(\frac{3}{2}+L_{1}\right)\left[2\left(\lambda_{p s}^{\not B L}\right)^{*}\left(\lambda_{r t}^{1 \mathrm{~L}}\right)+\left(\lambda_{r s}^{\not B L}\right)^{*}\left(\lambda_{t p}^{1 \mathrm{~L}}\right)-\left(\lambda_{p r}^{\not B L}\right)^{*}\left(\lambda_{s t}^{1 \mathrm{~L}}\right)\right], \\
{\left[G_{d u u}\right]_{p r s t}^{(1)}=} & {\left[\left(1+N_{c}\right)\left(1+L_{1}\right) c_{1}+\frac{2 \tilde{M}_{2}^{2}}{M_{1}^{2}}\left(1+L_{2}\right)\left(N_{c} c_{1 \tilde{2}}^{(1)}+c_{1 \tilde{2}}^{(2)}\right)\right.} \\
& -\left[4\left(1+L_{1}\right) g^{\prime 2}\left(2 Y_{u}^{2}-2 Y_{e} \frac{1}{M_{1}^{2}} L_{2}\right] \frac{\left(\lambda_{p r}^{\not B R}\right)^{*}\left(\lambda_{s t}^{1 \mathrm{R}}\right)}{M_{1}^{2}}\right. \\
& \left.+2 g^{\prime 2} Y_{u}\left(Y_{d}+3 Y_{S_{1}}\right)-2 g^{\prime 2} Y_{e}\left(3 Y_{d}-Y_{u}\right)\right] \frac{\left(\lambda_{p r}^{\not B R}\right)^{*}\left(\lambda_{s t}^{1 \mathrm{R}}\right)}{4 M_{1}^{2}}
\end{aligned}
$$




$$
\begin{aligned}
& -2 g^{\prime 2} \frac{\left(\lambda_{p s}^{\not B R}\right)^{*}\left(\lambda_{r t}^{1 \mathrm{R}}\right)}{M_{1}^{2}}\left(\frac{3}{2}+L_{1}\right)\left[Y_{d}\left(Y_{e}-Y_{u}\right)-Y_{u}\left(Y_{e}+Y_{u}\right)\right] \\
& -g_{s}^{2} \frac{1}{2}\left(\frac{7}{3}+L_{1}\right) \frac{\left(\lambda_{p r}^{\not B R}\right)^{*}\left(\lambda_{s t}^{1 \mathrm{R}}\right)}{M_{1}^{2}} .
\end{aligned}
$$

In total, 109 out of 139 operators are generated in the Green basis which translates into 53 out of 59 operators in the Warsaw basis. The only operators not generated by the $S_{1}+\tilde{S}_{2^{-}}$ model in the Warsaw basis are the $\mathrm{CP}$ violating ones, namely $Q_{3(\tilde{G}, \tilde{W}), H(\tilde{B}, \tilde{W}, \tilde{G}), H \tilde{W} B}$, which are of course absent in the Green basis as well.

The UOLEA parameters $\tilde{f}_{11}-\tilde{f}_{19}$ appearing in Vector-Bosons-Scalar operators are given separately in appendix D. The hypercharges of the SM chiral fermions and the Higgs are,

$$
Y_{\ell}=-\frac{1}{2}, \quad Y_{e}=-1, \quad Y_{q}=\frac{1}{6}, \quad Y_{u}=\frac{2}{3}, \quad Y_{d}=-\frac{1}{3}, \quad \text { and } \quad Y_{H}=\frac{1}{2},
$$

respectively, while $Y_{S_{1}}=1 / 3$ and $Y_{\tilde{S}_{2}}=1 / 6$ for leptoquarks.

\subsection{Theoretical remarks}

Further remarks on our findings for the complete set of $d \leq 6$ Wilson coefficients in Green basis at one-loop are in order.

\subsubsection{Evanescent operators}

Evanescent operators appear in 4-point functions involving fermions. Treating the integrals in $d$-dimensions while using Fierz identities, that hold only in $d=4$ or encountering higher order of $\gamma$-matrices products, give rise to evanescent operators that in general vanish in $d=4$. The scheme we will be using for this type of structures is the introduction of local counterterms $a_{e v}, \ldots, f_{e v}$. For details the reader is referred to refs. [45-48, 63].

Although, strictly-speaking, not part of the actual matching calculation, in translating the raw results of the traces after substituting the $U$ matrices, one needs to choose a definite scheme to reduce the $\gamma$-matrix structure appearing in the subsequent equations and match it to a certain basis, such as the Green basis. In the model under consideration evanescent operators make their appearance in the Supertrace of (2.35) where both left and right projection operators appear in the same trace. We keep a general parameter $a_{e v}$ not resorting to any particular scheme. The usual scheme choice for evanescent operators, however, is $a_{e v}=\ldots=f_{e v}=1$. The relevant Dirac-structures appearing in the model at hand are (in the NDR scheme $d=4-\epsilon$ ),

$$
\begin{aligned}
& P_{L} \gamma_{\mu} \gamma_{\nu} P_{L} \otimes P_{L} \gamma^{\mu} \gamma^{\nu} P_{L}=4\left(1-\frac{\epsilon}{4}\right) P_{L} \otimes P_{L}-P_{L} \sigma_{\mu \nu} P_{L} \otimes P_{L} \sigma^{\mu \nu} P_{L}, \\
& P_{L} \gamma_{\mu} \gamma_{\nu} P_{L} \otimes P_{L} \gamma^{\nu} \gamma^{\mu} P_{L}=4\left(1-\frac{\epsilon}{4}\right) P_{L} \otimes P_{L}+P_{L} \sigma_{\mu \nu} P_{L} \otimes P_{L} \sigma^{\mu \nu} P_{L}, \\
& P_{L} \gamma^{\mu} \gamma^{\nu} P_{L} \otimes P_{R} \gamma^{\mu} \gamma^{\nu} P_{R}=4\left(1+a_{e v} \frac{\epsilon}{2}\right) P_{L} \otimes P_{R}+E_{L R}^{(2)}, \\
& P_{L} \gamma^{\mu} \gamma^{\nu} P_{L} \otimes P_{R} \gamma^{\nu} \gamma^{\mu} P_{R}=4\left[1-\frac{\epsilon}{2}\left(1+a_{e v}\right)\right] P_{L} \otimes P_{R}+E_{L R}^{(2)} .
\end{aligned}
$$

Plugging in a specific value for the coefficient $a_{e v}$, in our case, defines the evanescent operators $E_{L R}^{(2)}$. 


\subsubsection{RGE checks}

As a further cross check of our results for Wilson coefficients we have calculated the Renormalization Group Equations (RGEs) for a certain set. For purely one-loop generated operators one has to just take the derivative with respect to the renormalization scale $\mu$ and extract the relevant $\beta$-function. For instance explicitly taking the derivative with respect to $\mu$ on $\left[C_{e W}\right]_{p r}$ we find,

$$
\frac{d\left[C_{e W}\right]_{p r}}{d \ln \mu}=-N_{c} \frac{g}{4} \frac{\left(Y_{1 U}^{1 L}\right)_{p r}}{M_{1}^{2}} .
$$

Comparing with the $\beta$-functions from [64-67],

$$
\left[\beta_{e W}\right]_{p r}=6 g\left(y_{U}\right)_{s t}^{*}\left[C_{\text {lequ }}^{(3)}\right]_{p r s t},
$$

after plugging in the value of the relevant Wc [eq. (3.14)], we get,

$$
\left[\beta_{e W}\right]_{p r}=-\frac{3 g}{4} \frac{\left(Y_{1 U}^{1 L}\right)_{p r}}{M_{1}^{2}},
$$

which is in complete agreement, for $N_{c}=3$, with the direct application of the derivative. The same procedure has been followed for every other purely one-loop generated Wcs and we have found no discrepancies in the comparison.

For operators generated at tree-level as well the picture is a bit different because at tree-level the coupling of the respective Wcs must be considered as running parameters due to shifts of the corresponding fields. However these exact shifts will cancel with the RG running due to wavefunction renormalization. For example, to bring back the lepton and quark doublet kinetic term we must make the following shift,

$$
\begin{gathered}
\ell_{p} \longrightarrow \ell_{p}-\frac{1}{2}\left(\delta Z_{\ell}\right)_{p p_{1}} \ell_{p_{1}}, \\
q_{p} \longrightarrow q_{p}-\frac{1}{2}\left(\delta Z_{q}\right)_{p p_{1}} q_{p_{1}},
\end{gathered}
$$

where $\delta Z_{(\ell, q)}$ correspond to eqs. (3.43) and (3.45), respectively. We have also suppressed all other indices apart from generation indices. In turn this produces a shift in the coupling $\lambda_{p r}^{1 \mathrm{~L}}$. To absorb this shift we redefine the coupling as,

$$
\left(\lambda_{p r}^{1 \mathrm{~L}}\right)^{\mathrm{eff} .}=\lambda_{p r}^{1 \mathrm{~L}}-\frac{1}{2} \lambda_{p w}^{1 \mathrm{~L}}\left(\delta Z_{\ell}\right)_{w r}-\frac{1}{2} \lambda_{w r}^{1 \mathrm{~L}}\left(\delta Z_{q}\right)_{w p} .
$$

Taking the derivative w.r.t. $\mu$-parameter we find the $\beta$-function for the effective running coupling,

$$
\begin{aligned}
{\left[\beta_{\lambda 1 \mathrm{~L}}\right]_{p r}=} & \frac{N_{c}}{2}\left[\lambda^{1 \mathrm{~L}}\left(\lambda^{1 \mathrm{~L}}\right)^{\dagger} \lambda^{1 \mathrm{~L}}+\lambda^{1 \mathrm{~L}}(\tilde{\lambda})^{\dagger} \tilde{\lambda}\right]_{p r} \\
& +\frac{1}{2}\left[\left(\lambda^{1 \mathrm{~L}}\right)^{T}\left(\lambda^{1 \mathrm{~L}}\right)^{*}\left(\lambda^{1 \mathrm{~L}}\right)^{T}-8\left(\lambda^{1 \mathrm{~L}}\right)^{T} \lambda^{\not B L}\left(\lambda^{\not B L}\right)^{\dagger}\right]_{p r} .
\end{aligned}
$$

The same of course can be done for the complex conjugate coupling,

$$
\left[\beta_{\left(\lambda^{1 \mathrm{~L}}\right)^{\dagger}}\right]_{p r}=\left[\beta_{\lambda^{1 \mathrm{~L}}}\right]_{p r}^{\dagger} .
$$


These two will contribute, for instance, in the RG evolution of the operator $C_{\ell q}^{(1)}$. Ignoring finite parts, and for the clarity of the cancellation considering only the part produced by the shifts in the fermion fields, we find

$$
\begin{aligned}
& {\left[C_{\ell q}^{(1)}\right]_{p r s t} \propto\left[C_{\ell q}^{(1)^{(0)}}\right]_{p r s t}^{(0)}-\frac{1}{2}\left\{\left(\delta Z_{\ell}\right)_{p w}\left[C_{\ell q}^{(1)^{(0)}}\right]_{w r s t}^{(0)}+\left(\delta Z_{\ell}\right)_{r w}\left[C_{\ell q}^{(1)}\right]_{p w s t}^{(0)}\right.} \\
& \left.+\left(\delta Z_{q}\right)_{s w}\left[C_{\ell q}^{(1)}\right]_{p r w t}^{(0)}+\left(\delta Z_{q}\right)_{t w}\left[C_{\ell q}^{(1)^{(1)}}\right]_{p r s w}^{(0)}\right\} \\
& +\left[c_{1} L_{1}+\frac{\tilde{M}_{2}^{2}}{2 M_{1}^{2}} L_{2}\left(3 c_{1 \tilde{2}}^{(1)}+c_{1 \tilde{2}}^{(2)}\right)+8\left|A^{\prime}\right|^{2} \frac{1}{M_{1}^{2}} L_{2}\right] \frac{\left(\lambda_{p s}^{1 \mathrm{~L}}\right)^{\dagger}\left(\lambda_{t r}^{1 \mathrm{~L}}\right)}{M_{1}^{2}} .
\end{aligned}
$$

Schematically for the operator above we have,

$$
\begin{aligned}
\frac{d\left[C_{\ell q}^{(1)}\right]_{p r s t}}{d \ln \mu} \propto & \frac{d\left[C_{\ell q}^{(1)}\right]_{p r s t}^{(0)}}{d \ln \mu}-\frac{1}{2} \frac{d}{d \ln \mu}\left\{\left(\delta Z_{\ell}\right)_{p w}\left[C_{\ell q}^{(1)}\right]_{w r s t}^{(0)}+\left(\delta Z_{\ell}\right)_{r w}\left[C_{\ell q}^{(1)^{(1)}}\right]_{p w s t}^{(0)}\right. \\
& +\left(\delta Z_{q}\right)_{s w}\left[C_{\ell q}^{\left.(1)^{(1)}\right]_{p r w t}}+\left(\delta Z_{q}\right)_{t w}\left[C_{\ell q}^{(1)}\right]_{p r s w}^{(0)}\right\} \\
& +\left[2 c_{1}+\frac{\tilde{M}_{2}^{2}}{M_{1}^{2}}\left(3 c_{1 \tilde{2}}^{(1)}+c_{1 \tilde{2}}^{(2)}\right)+16\left|A^{\prime}\right|^{2} \frac{1}{M_{1}^{2}}\right] \frac{\left(\lambda_{p s}^{1 \mathrm{~L}}\right)^{\dagger}\left(\lambda_{t r}^{1 \mathrm{~L}}\right)}{M_{1}^{2}} .
\end{aligned}
$$

On the other hand the derivative of the tree-level operator reads,

$$
\frac{d\left[C_{\ell q}^{(1)}\right]_{p r s t}^{(0)}}{d \ln \mu}=\frac{\left(\lambda_{p s}^{1 \mathrm{~L}}\right)^{\dagger}}{4 M_{1}^{2}}\left(\beta_{\lambda^{1 \mathrm{~L}}}\right)_{t r}+\frac{\left(\lambda^{1 \mathrm{~L}}\right)_{t r}}{4 M_{1}^{2}}\left(\beta_{\left(\lambda^{1 \mathrm{~L}}\right)^{\dagger}}\right)_{p s}-\frac{\left(\lambda_{p s}^{1 \mathrm{~L}}\right)^{\dagger}\left(\lambda^{1 \mathrm{~L}}\right)_{t r}}{4 M_{1}^{2}} \gamma_{M_{1}^{2}}
$$

where $\gamma_{M_{1}^{2}}=d \ln M_{1}^{2} / d \ln \mu$ is the anomalous dimension of the leptoquark mass. The last term in (3.183) will cancel with the last line of (3.182). In contrast to the coupling, this cancellation is not captured by the matching procedure, where we have assumed no heavy-external-field legs.

The remaining terms inside the curly brackets in (3.182), due to the redefintion of the fermion fields, exactly cancel the contributions to the $\beta$-function from the tree-level result (3.183). Cancellations aside, by taking the explicit derivative of the whole set of Wcs we can cross-check the logarithmic part of our results. Comparing the $\beta$-functions produced by our model, with the relevant parts of [64-67], we find complete agreement.

\subsection{Phenomenological aspects}

In this section we present some interesting phenomenological aspects arising from the $S_{1}+$ $\tilde{S}_{2}$ LQ-model whose one-loop effective Lagrangian derived previously in this section.

\subsubsection{Lepton magnetic and electric dipole moments}

There is a recent excitement about the muon anomalous magnetic moment. FNAL experiment [68] confirmed previous results by BNL experiment [69] and found a $4.2 \sigma$ excess w.r.t. the SM, $\Delta a_{\mu}=a_{\mu}^{\exp }-a_{\mu}^{\mathrm{SM}}=(251 \pm 59) \times 10^{-11}[68]$. As a demonstration of our one-loop effective Lagrangian we work out the contribution to $\Delta a_{\mu}$ from the decoupling of $S_{1}+\tilde{S}_{2}$-leptoquarks, and compare it with the fixed order one-loop calculation. 
Within functional approach, contributions to magnetic moments of fermions arise from eqs. (2.58) and (2.59). The corresponding functional supertrace diagram and its expression is displayed in (2.31). The above contributions along with the insertion of the tree-level operator $\left[\mathcal{O}_{\text {lequ }}^{(3)}\right]$, associated with $\left[C_{\text {lequ }}^{(3)}\right]$ given in eq. (3.14), in a one-loop diagram computed for example in ref. [70] constitute the full EFT formula in this model. The dominant new physics contributions to the anomalous magnetic moment of the $\ell$-generation lepton are thus

$$
\Delta a^{\ell}=\frac{4 m_{\ell} v}{\sqrt{2}}\left[\frac{1}{g^{\prime}} \Re e\left[\mathcal{C}_{e B}\right]-\frac{1}{g} \Re e\left[\mathcal{C}_{e W}\right]\right]_{\ell \ell}+\frac{2 N_{c}}{3 \pi^{2}} \sum_{q} m_{\ell} m_{q} \log \left(\frac{\mu^{2}}{m_{q}^{2}}\right) \Re e\left\{\left[\mathcal{C}_{\ell e q u}^{(3)}\right]_{\ell \ell q q}\right\},
$$

where $v$ is the vev, $m_{\ell}$ is the $\ell=e, \mu, \tau$ lepton mass and $m_{q}$ is the quark mass running in the loop with $q=t, c, u$. We note for later, that when evolving down to the top quark mass, $m_{t}$, the dominant part of the last term vanishes, hence we can neglect all other sub-leading terms in the sum. Therefore, we are left with the first two terms in the square bracket. The coefficients $\mathcal{C}_{e B}$ and $\mathcal{C}_{e W}$ are defined at low energies in mass basis of ref. [57]. They are related to the Warsaw gauge basis coefficients, $C_{e B}$ and $C_{e W}$, through the expressions,

$$
\mathcal{C}_{e B}=U_{e_{L}}^{\dagger} C_{e B} U_{e_{R}}, \quad \mathcal{C}_{e W}=U_{e_{L}}^{\dagger} C_{e W} U_{e_{R}},
$$

where the unitary matrices $U_{e_{L, R}}$ diagonalize the lepton mass matrices, $U_{e_{L}}^{\dagger} y_{E} U_{e_{R}}=\hat{y}_{E}=$ $\operatorname{diag}\left(y_{e}, y_{\mu}, y_{\tau}\right)$. Since our results are given in Green basis we need a translation from Green to Warsaw basis. This translation is nicely given in ref. [33]. After a little algebra, we find the coefficients at renormalization scale $\mu$ (still gauge basis in $3 \times 3$ matrix notation) to be

$$
\begin{gathered}
{\left[C_{e B}\right]^{(1)}(\mu)=\frac{g^{\prime} N_{c}}{16 \pi^{2}}\left\{\frac{5}{24}\left[\log \left(\frac{\mu^{2}}{M_{1}^{2}}\right)+\frac{19}{10}\right] \frac{Y_{1 U}^{1 L}}{M_{1}^{2}}-\frac{1}{24} \frac{y_{E} \cdot \Lambda_{e}}{M_{1}^{2}}-\frac{1}{48} \frac{\tilde{\Lambda}_{\ell} \cdot y_{E}}{\tilde{M}_{2}^{2}}\right\},} \\
{\left[C_{e W}\right]^{(1)}(\mu)=\frac{g N_{c}}{16 \pi^{2}}\left\{-\frac{1}{8}\left[\log \left(\frac{\mu^{2}}{M_{1}^{2}}\right)+\frac{3}{2}\right] \frac{Y_{1 U}^{1 L}}{M_{1}^{2}}+\frac{1}{24} \frac{\Lambda_{\ell} \cdot y_{E}}{M_{1}^{2}}-\frac{1}{48} \frac{\tilde{\Lambda}_{\ell} \cdot y_{E}}{\tilde{M}_{2}^{2}}\right\},}
\end{gathered}
$$

where ". " means matrix multiplication. These results are in agreement with ref. [70]. The parameters $Y_{1 U}^{1 L}, \Lambda_{e}, \tilde{\Lambda}_{\ell}$ and $\Lambda_{\ell}$ are defined in eqs. (3.31), (3.29) and (3.28), respectively. We then use the RGE running of the coefficients $C_{e B}, C_{e W}$ from the heavy leptoquark mass scale $M_{1}$ down to the top-quark mass scale $m_{t}$ and plug the result into (3.184) to find at leading-log approximation (for $N_{c}=3$ ): ${ }^{6}$

$$
\begin{aligned}
\Delta a_{\ell}^{\left(S_{1}+\tilde{S}_{2}\right)}= & \sum_{q=u, c, t} \frac{m_{\ell}}{4 \pi^{2}} \frac{m_{q}}{M_{1}^{2}}\left[\log \left(\frac{m_{t}^{2}}{M_{1}^{2}}\right)+\frac{7}{4}\right] \Re e\left(\hat{\lambda}_{q \ell}^{1 L *} \hat{\lambda}_{q \ell}^{1 R}\right) \\
& -\frac{m_{\ell}^{2}}{32 \pi^{2} M_{1}^{2}}\left(\hat{\lambda}_{q \ell}^{1 L} \hat{\lambda}_{q \ell}^{1 L}+\hat{\lambda}_{q \ell}^{1 R *} \hat{\lambda}_{q \ell}^{1 R}\right)
\end{aligned}
$$

where all parameters and masses are to be evaluated at $m_{t}$ and the "hatted" couplings are defined in mass basis as,

$$
\hat{\lambda}^{1 L}=U_{u_{L}}^{T} \lambda^{1 L} U_{e_{L}}, \quad \hat{\lambda}^{1 R}=U_{u_{R}}^{T} \lambda^{1 R} U_{e_{R}}, \quad \hat{\tilde{\lambda}}=U_{d_{R}}^{\dagger} \tilde{\lambda} U_{e L},
$$

\footnotetext{
${ }^{6}$ To leading-log order the result is the same by setting $\mu=m_{t}$ in (3.186) and (3.187) and then take the difference in (3.184), and neglecting the contribution from light quark masses.
} 
with $U_{u_{L}}^{\dagger} y_{U} U_{u_{R}}=\hat{y}_{U}=\operatorname{diag}\left(y_{u}, y_{s}, y_{t}\right)$ being the up-quark fermion Yukawa couplings. Note that, as it should, the one-loop expression (3.188) agrees with the fixed order calculation of ref. [39] for the $S_{1}$-leptoquark decoupling. Also obvious from (3.188) is a natural enhancement of $O\left(m_{t} / m_{\ell}\right)$ due to $S_{1}$-decoupling, while there is no effect from the $\tilde{S}_{2}$-particle decoupling. However, a similar enhancement is shown in eq. (3.49), for the one-loop corrections to the Yukawa coupling of the leptons, and subsequently to the lepton mass itself.

Moreover, a bound on electron Electric Dipole Moment (eEDM), $\left|d_{e}\right|<1.1 \times$ $10^{-29} \mathrm{e} \cdot \mathrm{cm}$ at $90 \% \mathrm{CL}$, anounced by ACME collaboration [71] almost three years ago. Our complete 1-loop functional matching LQs renders the calculation of eEDM very easy. As mentioned previously, in the model at hand $\left(S_{1}+\tilde{S}_{2}\right)$, bosonic operators of the form $H^{2} F \tilde{F}$ and $F^{3}$ are not induced, therefore the only effect at one-loop arises from the Warsawbasis operators $Q_{e B}, Q_{e W}$ and $Q_{\ell e q u}^{(3)}$ as before. Basically, the calculation is the same with the lepton magnetic moments we performed above. Again, neglecting the contribution from $C_{\text {lequ }}^{(3)}$ since the evaluation is at $\mu=m_{t}$, the eEDM reads,

$$
\frac{d_{e}}{e}\left(m_{t}\right)=\sqrt{2} v\left[\frac{1}{g^{\prime}} \Im m\left[\mathcal{C}_{e B}\left(m_{t}\right)\right]-\frac{1}{g} \Im m\left[\mathcal{C}_{e W}\left(m_{t}\right)\right]\right]_{11} .
$$

Plugging into (3.190) the imaginary parts of eqs. (3.186) and (3.187) after substituting $\mu=m_{t}$, we find

$$
\frac{d_{e}}{e}=\frac{1}{8 \pi^{2}} \frac{m_{t}}{M_{1}^{2}}\left[\log \left(\frac{m_{t}^{2}}{M_{1}^{2}}\right)+\frac{7}{4}\right] \Im m\left(\hat{\lambda}_{31}^{1 L *} \hat{\lambda}_{31}^{1 R}\right)
$$

This result agrees with the fixed order 1-loop calculation, up to $\mathcal{O}\left(m_{t}^{2} / M_{1}^{4}\right)$-terms, obtained by applying the general one-loop formula for lepton EDMs from ref. [72] onto the particular $S_{1}+\tilde{S}_{2}$ LQ-model. The leading-log term of (3.191) also agrees with the one obtained in refs. [38, 73].

The reader should note that this is a SMEFT calculation, i.e. we have chosen $\mu=m_{t}$. Ideally it's more appropriate to match onto Low Energy Effective Field Theory (LEFT) and perform the calculation of dipole moments at lower scales, which is beyond the scope of this paper. For a thorough EFT analysis of leptonic magnetic and electric dipole moments along these lines the reader is referred to refs. [70, 74].

\subsubsection{Radiative neutrino masses}

A nice feature of the $S_{1}+\tilde{S}_{2}$ model is that neutrino masses are induced radiatively at oneloop. The single dimension-5 operator $Q_{\nu \nu}$, defined in appendix C, arises in the effective Lagrangian from the supertrace functional diagram (2.34) which after calculation provides us the associated Wilson coefficient $G_{\nu \nu}=C_{\nu \nu}$ (3.54) in both Green and Warsaw basis. The result is finite and agrees with ref. [41]. Then going to the mass basis SMEFT Lagrangian of ref. [57] we have for the diagonal neutrino mass matrix

$$
m_{\nu}=-v^{2} U_{\nu_{L}}^{T} C_{\nu \nu} U_{\nu_{L}} .
$$


By using the tree level definitions of CKM and MNS matrices, ${ }^{7}$ as $K_{\mathrm{CKM}}=U_{u_{L}}^{\dagger} U_{d_{L}}$ and $U_{\mathrm{MNS}}=U_{e_{L}}^{\dagger} U_{\nu_{L}}$ and eq. (3.189) we obtain

$$
m_{\nu}=-\frac{\sqrt{2}}{16 \pi^{2}} \frac{v A_{\tilde{2} 1}}{M_{1}^{2}-\tilde{M}_{2}^{2}}\left[U_{\mathrm{MNS}}^{T}\left(\hat{\lambda}^{1 L}\right)^{T} K_{\mathrm{CKM}} m_{d} \hat{\tilde{\lambda}} U_{\mathrm{MNS}}\right] \log \left(\frac{M_{1}^{2}}{\tilde{M}_{2}^{2}}\right),
$$

where $m_{d}$ is the diagonal down quark mass matrix. The neutrino masses clearly follow a "down-quark" mass hierarchy with the couplings $\hat{\lambda}^{1 L}, \hat{\tilde{\lambda}}$ and the CKM matrix defining off-diagonal transitions. Obviously, depending on the value of the parameter $A_{\tilde{2} 1}$ that mixes both leptoquarks with the Higgs boson, we can probe two different mass scales: (i) $A_{\tilde{2} 1} / \max \left(M_{1}, M_{2}\right) \simeq 1$, and (ii) $A_{\tilde{2} 1} / \max \left(M_{1}, M_{2}\right) \rightarrow 0$. In case (i) correct order of magnitude of neutrino mass, $m_{\nu} \lesssim 0.1 \mathrm{eV}$, and $\lambda \sim O(1)$ requires $M_{1} \approx M_{2} \gtrsim 10^{13} \mathrm{GeV}$ and this is currently consistent with proton decay bounds (see below), whereas in case (ii) $M_{1} \approx M_{2} \simeq 1 \mathrm{TeV}$ requires $A_{\tilde{2} 1} \approx 10^{-7} \mathrm{GeV}$ which is technically natural in terms of a $\mathbb{Z}_{3}$ $\left(\right.$ or $\mathbb{Z}_{4}$ ) softly broken discrete symmetry discussed in section 3 (but still baryon number violating couplings, $\lambda^{\not B L}$ and $\lambda^{\not B R}$, are allowed and have to be set to zero by another symmetry in order to avoid fast proton decay).

\subsubsection{Proton decay}

Baryon number is violated in the $S_{1}+\tilde{S}_{2}$ model. All $d=6$ baryon number violating (BNV) operators appear already at tree level in the effective Lagrangian [eqs. (3.19), (3.20)], after the decoupling of $S_{1}$-field. From these expressions and table 3 we easily see they have $\Delta B=\Delta L=1$ consistent with eqs. (3.24) and (3.25). Rotating fermion fields into the mass basis of ref. [57] we find for the tree-level Wilson-coefficients

$$
\begin{aligned}
{\left[\mathcal{C}^{q q u}\right]_{f_{1} f_{2} f_{3} f_{4}}^{(0)} } & =\frac{1}{M_{1}^{2}}\left[K_{\mathrm{CKM}}^{T}\left(\lambda^{\hat{B} L}\right)^{*}\right]_{f_{1} f_{2}}\left(\hat{\lambda}^{1 R}\right)_{f_{3} f_{4}}, \\
{\left[\mathcal{C}^{d u q}\right]_{f_{1} f_{2} f_{3} f_{4}}^{(0)} } & =\frac{1}{M_{1}^{2}}\left(\lambda^{\hat{B} R}\right)_{f_{1} f_{2}}^{*}\left[K_{\mathrm{CKM}}^{T} \hat{\lambda}^{1 L}\right]_{f_{3} f_{4}}, \\
{\left[\mathcal{C}^{d u u}\right]_{f_{1} f_{2} f_{3} f_{4}}^{(0)} } & =\frac{1}{M_{1}^{2}}\left(\lambda^{\hat{B} R}\right)_{f_{1} f_{2}}^{*}\left(\hat{\lambda}^{1 R}\right)_{f_{3} f_{4}}, \\
{\left[\mathcal{C}^{q q q}\right]_{f_{1} f_{2} f_{3} f_{4}}^{(0)} } & =-\frac{2}{M_{1}^{2}}\left[K_{\mathrm{CKM}}^{T}\left(\lambda^{\hat{B} L}\right)^{*}\right]_{f_{1} f_{2}}\left[K_{\mathrm{CKM}}^{T} \hat{\lambda}^{1 L}\right]_{f_{3} f_{4}}
\end{aligned}
$$

where the "hatted" couplings are given in eq. (3.189) in addition to

$$
\lambda^{\hat{B} L}=U_{u_{L}}^{\dagger} \lambda^{\not B L} U_{d_{L}}^{*}, \quad \lambda^{\hat{B} R}=U_{d_{R}}^{\dagger} \lambda^{\not B R} U_{u_{R}}^{*} .
$$

Obviously, due to lepton and baryon quantum numbers arranged in table 3, only one BNVcoupling, $\lambda^{\not B L}$ or $\lambda^{\not B R}$, appear for $\Delta L=\Delta B=1$. Plugging these into the Feynman Rules of ref. [57] we derive decay rates for general nucleon decay processes.

To date, proton decay has not been observed and Super Kamiokande has increased the proton lifetime limits up to $\sim 10^{34}$ years with bounds [75, 76]

$$
\tau\left(p \rightarrow e^{+} \pi^{0}\right)>1.6 \times 10^{34} \mathrm{yrs}, \quad \tau\left(p \rightarrow \bar{\nu} K^{+}\right)>0.59 \times 10^{34} \mathrm{yrs},
$$

\footnotetext{
${ }^{7}$ There is no "pollution" to CKM or MNS matrices from other tree level operators in this model.
} 
being the most sensitive ones to BSM physics [77]. Based on the first of these bounds we derive constraints on the following products of couplings:

$$
2\left(\lambda^{\hat{B} R}\right)_{11}^{*} \hat{\lambda}_{11}^{1 R}, \quad 2\left(\lambda^{\hat{B} R}\right)_{11}^{*} \hat{\lambda}_{11}^{1 L}, \quad 2\left(\lambda^{\hat{B} L}\right)_{11}^{*} \hat{\lambda}_{11}^{1 R}, \quad 4\left(\lambda^{\hat{B}} L\right)_{11}^{*} \hat{\lambda}_{11}^{1 L} \lesssim 10^{-6}\left(\frac{M_{1}}{3 \times 10^{12}}\right)^{2} .
$$

Note that although the CKM-matrix, $K_{\mathrm{CKM}}$, appears explicitly in tree expressions (3.194)(3.197), it disappears completely from the relevant BNV vertices of ref. [57] for physical fields. In summary, $M_{1} \gtrsim 3 \times 10^{12} \mathrm{GeV}$ and first generation $\hat{\lambda}^{1 L, 1 R} \sim O(1)$ and $\lambda^{\not B L}$ and $\lambda^{\not B R}$ of the order of electron yukawa coupling is, currently, a safe combination.

At tree level, the proton decay constraints in eq. (3.200) apply on the 11 entries of $\lambda$-matrices. This changes by going to higher orders where in principle all flavour structure of those matrices are getting involved through the CKM-matrix. One loop contributions to BNV Wilson coefficients are given in eqs. (3.164)-(3.167). For example, by just looking at $\left[G_{q q q}\right]^{(1)}$ and the term proportional to the strong QCD coupling, $g_{s}^{2}\left(\lambda_{p s}^{\not B L}\right)^{*}\left(\lambda_{s t}^{1 \mathrm{~L}}\right)$ we find a contribution to the $p \rightarrow e^{+} \pi^{0}$ amplitude of the form $g_{s}^{2}\left(\lambda^{\hat{B} L} K_{\mathrm{CKM}}^{\dagger}\right)_{11}\left(K_{C K M}^{T} \hat{\lambda}^{1 L}\right)_{11}$ which, although CKM-suppressed, displays a certain sensitivity in the off-diagonal $\lambda_{1 i}$ entries under the strong proton decay bounds of (3.199). A partial list of dedicated studies on proton decay in leptoquark-like models are given in refs. [51, 78-80] and related reviews in refs. $[52,77]$.

In summary, unless there is a symmetry to prohibit BNV-couplings, the mass $M_{1}$ must be bigger than the "intermediate" scale $\sim 10^{12} \mathrm{GeV}$. As we discuss in the next paragraph, this will bring every other leptoquark masses e.g. $\tilde{M}_{2}$, at around that scale unless unnatural fine tuning is called upon in the Higgs sector.

\subsubsection{Perturbativity and fine tuning}

An interesting interplay between the LQ masses can be explored in some operators that contain the ratio of the two masses. We can thus put some bounds on the ratio $M_{1} / \tilde{M}_{2}$ so that perturbation theory is not violated. For example, for the operator $G_{\ell d}$ adding the tree, eq. (3.15), and one-loop level, eq. (3.160) that depends on the LQ mass ratio, we have,

$$
\left[G_{\ell d}\right]_{p r s t} \propto-\frac{\tilde{\lambda}_{t p}^{*} \tilde{\lambda}_{s r}}{2 \tilde{M}_{2}^{2}}\left(1+\frac{1}{16 \pi^{2}} \frac{M_{1}^{2}}{\tilde{M}_{2}^{2}}\left(N_{c} c_{1 \tilde{2}}^{(1)}+c_{1 \tilde{2}}^{(2)}\right)\left(1+L_{1}\right)\right) .
$$

Other operators depend on the inverse mass ratio $\tilde{M}_{2} / M_{1}$. For instance, adding eq. (3.16) and eq. (3.139), we have,

$$
\left[G_{\ell q}^{(1)}\right]_{p r s t} \propto \frac{\left(\lambda^{1 \mathrm{~L}}\right)_{s p}^{*}\left(\lambda_{t r}^{1 \mathrm{~L}}\right)}{4 M_{1}^{2}}\left(1+\frac{2}{16 \pi^{2}} \frac{\tilde{M}_{2}^{2}}{M_{1}^{2}}\left(N_{c} c_{1 \tilde{2}}^{(1)}+c_{1 \tilde{2}}^{(2)}\right)\left(1+L_{2}\right)\right) .
$$

For perturbation theory to work, loop level contributions have to be way smaller than tree level ones. Then we can get a combined constrain for the ratio of the masses,

$$
2 \frac{N_{c} c_{1 \tilde{2}}^{(1)}+c_{1 \tilde{2}}^{(2)}}{16 \pi^{2}}\left(1+L_{2}\right)<\frac{M_{1}^{2}}{\tilde{M}_{2}^{2}}<\frac{16 \pi^{2}}{\left(1+L_{1}\right)\left(N_{c} c_{1 \tilde{2}}^{(1)}+c_{1 \tilde{2}}^{(2)}\right)},
$$


which depends explicitly on parameters of the self interactions of the leptoquarks. Next, we define the following quantities,

$$
r^{2} \equiv \frac{M_{1}^{2}}{\tilde{M}_{2}^{2}}, \quad \alpha \equiv \frac{N_{c} c_{1 \tilde{2}}^{(1)}+c_{1 \tilde{2}}^{(2)}}{16 \pi^{2}} .
$$

The inequality then becomes,

$$
2 \alpha\left(1+L_{2}\right)<r^{2}<\frac{1}{\alpha\left(1+L_{1}\right)} .
$$

Which can be written in the following form,

$$
\frac{\mu^{2}}{\tilde{M}_{2}^{2}} \exp \left(1-\frac{1}{\alpha r^{2}}\right)<r^{2}<\frac{M_{1}^{2}}{\mu^{2}} \exp \left(\frac{r^{2}}{2 \alpha}-1\right) .
$$

From here on we will pick up a renormalization scale, first we will pick $\mu=M_{1}$ and then $\mu=\tilde{M}_{2}$. As for the parameters $c_{1 \tilde{2}}^{(1,2)}$ we will take them first of order unity and second to a fine tuned value of the order of $10^{-4}$. Plugging these numbers into eq. (3.204) we get $\alpha=1 / 4 \pi^{2}$ and $\alpha=10^{-4} / 4 \pi^{2}$ for each respective value of c's. Both these values will be used for both cases of the renormalization scale and extract some bounds for the masses.

- For the first case, $\mu=M_{1}$. In this case the combined inequality reads, for $N_{c}=3$,

$$
r^{2} \exp \left(1-\frac{1}{\alpha r^{2}}\right)<r^{2}<\exp \left(\frac{r^{2}}{2 \alpha}-1\right) .
$$

The right inequality is always satisfied, while from the left one we can get that,

$$
r^{2}<\frac{1}{\alpha}
$$

Considering the two distinct values of $\alpha$, we can get $M_{1}<\left(2 \pi, 2 \pi \times 10^{2}\right) \tilde{M}_{2}$.

- For the second case $\mu=\tilde{M}_{2}$. The inequality becomes,

$$
\exp \left(1-\frac{1}{\alpha r^{2}}\right)<r^{2}<r^{2} \exp \left(\frac{r^{2}}{2 \alpha}-1\right) \text {. }
$$

The right part gives, $r^{2}>2 \alpha$, while the left part is always true.

If we combine the two results we can get the following bound,

$$
2 \alpha<r^{2}<\frac{1}{\alpha} \text {. }
$$

Plugging the values of $\alpha$ we get,

$$
\frac{1}{\sqrt{2} \pi}\left(1,10^{-2}\right)<\frac{M_{1}}{\tilde{M}_{2}}<2 \pi\left(1,10^{2}\right) .
$$

Therefore, we can conclude that when couplings $c_{1 \tilde{2}}^{(1,2)}$ are of $\mathcal{O}(1)$ the masses can be taken to be of similar magnitude without violating perturbation theory. If we fine tune the 
couplings to even smaller values we can further increase the mass ratio giving us more room to tune the numerical values of the masses.

Noteworthy, the situation we just described for the Wcs, $G_{\ell d}^{(1)}$ and $G_{\ell q}^{(1)}$, share the same characteristics with the Higgs mass hierarchy problem which is of course evident in LQmodels. Indeed, by performing the matching procedure we have assumed that the Higgs mass $m$ is zero i.e., the Higgs field is part of the low energy EFT. Therefore, one-loop contributions to the Higgs mass found in (3.53) have to be of the order of the EW scale. For this to happen there are two cases (i) LQ-masses are of the order of the TeV-scale and Higgs couplings naturally of order $\mathcal{O}(1)$ or (ii) LQ-masses are heavier but Higgs couplings e.g. $\lambda_{H 1}, \tilde{\lambda}_{H 2}, \lambda_{\tilde{2} \tilde{2}}$ together with $A_{\tilde{2} 1} / M_{1}$ are small enough, although there is no known symmetry to naturally accommodate all these limits.

\section{Conclusions}

The resurgence of functional techniques to matching has led to a fair amount of universal results and compact formulae over the last few years. In this work we explored the matching of all scalar leptoquark representations that can be constructed under the SM gauge group. We have extracted through the use of Supertrace functional techniques [23] a universal formula, eq. (2.9) for tree level and eq. (2.43) for one-loop matching plus all $\mathbf{X}$-matrices in appendix A, for the decoupling of all scalar leptoquarks and put it to use in two distinct models. First we cross-tested it with the Feynman diagrammatic approach of the $S_{1}+S_{3}$ model [33]. Then we applied it to the, phenomenologically richer, $S_{1}+\tilde{S}_{2}$ model taking also baryon number violating couplings into account. In total, the latter model generates the single dimension-5 Weinberg operator at one loop, which gives rise to radiative neutrino masses. At dimension-6, 109 operators are generated in the Green basis while in the translation to Warsaw basis we are left with 53 (out of 59) operators thus covering almost the entire spectrum of dimension- 6 operators, the only exception being the set of 6 bosonic CP-violating ones. Our results of the given Wilson coefficients, derived in section 3 , have been also cross checked with the one loop RGEs finding complete agreement.

On the phenomenological side, we have briefly explored several distinct observables. First, we studied the implications to the lepton magnetic and electric dipole moments where there has been recent experimental advances. With this example we demonstrated the use of the matching in arriving at know results from fixed order calculations. Secondly, we have investigated possible regions of leptoquark masses, at the scale of a few $\mathrm{TeV}$ and at a high scale, as well as their coupling with the Higgs field, to generate radiatively the order of magnitude of neutrino masses through the Weinberg operator. Furthermore, we were able to put certain bounds in the combinations of BNV and non-BNV couplings, through the investigation of proton decay at tree level and one-loop. Last but not least, we have constructed a combined inequality for the ratio of the two LQ masses so that perturbation theory is not violated and briefly discussed the hierarchy problem in LQ-models.

As a concluding remark we would like to point out that models covering almost the entirety of the given operator basis spectrum, can serve as excellent benchmarks for various codes that will perform the matching automatically. The main reason for this argument 
is that within matching one needs to apply a fair amount of identities ranging from group theoretic, to Fierz identities and also accounting for evanescent operators arising from higher number of $\gamma$-matrix structures. All of the above have been encountered in the models that have been investigated in this work, ultimately, adding up to the number of fully worked out examples of one loop matching.

\section{Acknowledgments}

We would like to thank Valerio Gherardi for useful insights upon their work while comparing our results of the Wilson coefficients to theirs and for helping us catching several sign mistakes. We also thank Xiaochuan Lu for taking the time in writing useful comments on the use of STrEAM and clarifying some of its functions. We would like to thank the authors of refs. [81] and [70] each helping us to find a sign error in two Wilson coefficients. KM acknowledges funding from University of Ioannina Research Committee.

\section{A Lagrangian and X-matrices for scalar leptoquarks}

We append here the general Lagrangian for all scalar leptoquarks and the relevant $\mathbf{X}$ matrices needed to construct the EFT Lagrangian at one-loop. It can be split into three parts, in eqs. (2.1), (2.2) and (2.3). The notation for LQs is given in table 1.

The LQ-SM fermion interactions are,

$$
\begin{aligned}
\mathcal{L}_{\mathrm{LQ}-\mathrm{f}}= & {\left[\left(\lambda_{p r}^{1 \mathrm{~L}}\right) \bar{q}_{p i}^{c} \cdot \epsilon \cdot \ell_{r}+\left(\lambda_{p r}^{1 \mathrm{R}}\right) \bar{u}_{i}^{c} e_{r}\right] S_{1 i}+\text { h.c. } } \\
& +\left[\left(\lambda_{p r}^{\not B L}\right) \epsilon^{i j k} \bar{q}_{p j} \cdot \epsilon \cdot q_{r k}^{c}+\left(\lambda_{p r}^{\not B R}\right) \epsilon^{i j k} \bar{d}_{p j} u_{r k}^{c}\right] S_{1 i}+\text { h.c. } \\
& +\left[\left(\tilde{\lambda}_{p r}^{1}\right) \bar{d}_{p i}^{c} e_{r}+\left(\tilde{\lambda}_{p r}^{1 \not B}\right) \epsilon^{i j k} \bar{u}_{p j} u_{r k}^{c}\right] \tilde{S}_{1 i}+\text { h.c. } \\
& +\left[\left(\lambda_{p r}^{2 L R}\right) \bar{q}_{p i \alpha} e_{r}-\left(\lambda_{p r}^{2 R L}\right) \bar{u}_{p i} \ell_{r \beta} \epsilon^{\beta \alpha}\right] S_{2 i \alpha}+\text { h.c. } \\
& +\left(\tilde{\lambda}_{p r}\right) \bar{d}_{p i} \tilde{S}_{2 i}^{T} \cdot \epsilon \cdot \ell_{r}+\text { h.c. } \\
& +\left[\left(\lambda_{p r}^{3 L}\right) \bar{q}_{p i}^{c} \cdot \epsilon \cdot \sigma^{I} \cdot \ell_{r}+\left(\lambda_{p r}^{3 \not B}\right) \epsilon^{i j k} \bar{q}_{p j} \cdot \sigma^{I} \cdot \epsilon \cdot q_{r k}^{c}\right] S_{3 i}^{I}+\text { h.c. }
\end{aligned}
$$

The LQ-Higgs interactions read,

$$
\begin{aligned}
\mathcal{L}_{\mathrm{LQ}-\mathrm{H}}= & -\sum_{n}\left(M_{n}^{2}+\lambda_{H n}\left|S_{n}\right|^{2}\right)|H|^{2}+\sum_{n=2, \tilde{2}} \lambda_{n n}\left(S_{n i}^{\dagger} \cdot H\right)\left(H^{\dagger} \cdot S_{n i}\right) \\
& \times\left[-A_{\tilde{2} 1} S_{1 i}^{\dagger}\left(\tilde{S}_{2 i}^{\dagger} \cdot H\right)+A_{\tilde{2} 3} S_{3 i}^{I^{\dagger}}\left(\tilde{S}_{2 i}^{\dagger} \cdot \sigma^{I} \cdot H\right)\right. \\
& +\lambda_{2 \tilde{2}}\left(S_{2 i}^{\dagger} \cdot H\right)\left(H^{T} \cdot \epsilon \cdot \tilde{S}_{2 i}\right)+\lambda_{3 \tilde{1}} \tilde{S}_{1 i}^{\dagger}\left(H^{T} \cdot \epsilon \cdot \sigma^{I} \cdot H\right) S_{3 i}^{I} \\
& \left.+\lambda_{H 13}\left(H^{\dagger} \cdot \sigma^{I} \cdot H\right) S_{3 i}^{I \dagger} S_{1 i}+\text { h.c. }\right] \\
& -i \lambda_{\epsilon H 3} \epsilon^{I J K}\left(H^{\dagger} \cdot \sigma^{I} \cdot H\right) S_{3 i}^{J \dagger} S_{3 i}^{K \dagger}
\end{aligned}
$$

Where the index $n$ runs through, $n=1, \tilde{1}, 2, \tilde{2}, 3$ in one to one correspondence to the sets $\left\{S_{1}, \tilde{S}_{1}, S_{2}, \tilde{S}_{2}, S_{3}\right\},\left\{M_{1}, \tilde{M}_{1}, M_{2}, \tilde{M}_{2}, M_{3}\right\}$ and $\left\{\lambda_{H 1}, \tilde{\lambda}_{H 1}, \lambda_{H 2}, \tilde{\lambda}_{H 2}, \lambda_{H 3}\right\}$. 
Finally, self-interactions among scalar leptoquarks are

$$
\mathcal{L}_{\mathrm{S}}=-V(S),
$$

where $V(S)$ is the tree-level potential that is built from gauge group invariant combinations among LQ fields. In general $V(S)$ for all LQs is quite lengthy. For combinations $S_{1}+\tilde{S}_{2}$, the potential $V\left(S_{1}, \tilde{S}_{2}\right)$ can be read from (3.4), for $S_{1}+S_{3}$ from eq. (2.3) of ref. [33] while the most general one in eqs. (46) and (49) of ref. [82]. Note that $X(U)$-matrices are constructed solely from simple second field derivatives of $V(S)$ and there is no need to be written down explicitly.

In what follows we reserve letters $i, \alpha, p, \mu, A$ and $I$ to denote the respective field indices in the left hand side multiplet $\bar{\varphi}=\left(\bar{\varphi}_{S}, \bar{\varphi}_{L}\right)$, while the letters $j, \beta, r, \nu, B, J$ are used for the right hand side multiplet $\varphi=\left(\varphi_{S}, \varphi_{L}\right)$ and we suppress spinor indices. Each letter represents the respective gauge group representation given in table 1. Additionally, the chirality projection operators regarding the Weyl to Dirac conversion of fermions mentioned in the main text, are left implicit.

\section{A.1 $\mathbf{X}_{S S}$}

$\mathrm{U}_{\mathrm{S}_{1} \mathrm{~S}_{1}}$ :

$$
\begin{aligned}
& U_{S_{1}^{\dagger} S_{1}}=\lambda_{H 1}|H|^{2} \delta_{i j}+\frac{\partial^{2} V}{\partial S_{1 i}^{\dagger} \partial S_{1 j}}, \\
& U_{S_{1} S_{1}^{\dagger}}=\lambda_{H 1}|H|^{2} \delta_{i j}+\frac{\partial^{2} V}{\partial S_{1 i} \partial S_{1 j}^{\dagger}} .
\end{aligned}
$$

$\mathbf{U}_{\mathbf{S}_{1} \tilde{\mathbf{S}}_{2}}$ :

$$
\begin{gathered}
U_{S_{1} \tilde{S}_{2}}=A_{\tilde{2} 1}^{*} H_{\beta}^{*} \delta_{i j}+\frac{\partial^{2} V}{\partial S_{1 i} \partial \tilde{S}_{2 j \beta}}, \\
U_{S_{1}^{\dagger} \tilde{S}_{2}^{*}}=A_{21} H_{\beta} \delta_{i j}+\frac{\partial^{2} V}{\partial S_{1 i}^{\dagger} \partial \tilde{S}_{2 j \beta}^{*}} .
\end{gathered}
$$

$\mathrm{U}_{\mathrm{S}_{1} \mathrm{~S}_{3}}$ :

$$
\begin{gathered}
U_{S_{1}^{\dagger} S_{3}}=\lambda_{H 13}^{*} \delta_{i j}\left(H^{\dagger} \sigma^{J} H\right)+\frac{\partial^{2} V}{\partial S_{1 i}^{\dagger} \partial S_{3 j}^{J}}, \\
U_{S_{1} S_{3}^{*}}=\lambda_{H 13} \delta_{i j}\left(H^{\dagger} \sigma^{J} H\right)+\frac{\partial^{2} V}{\partial S_{1 i} \partial S_{3 j}^{I *}} .
\end{gathered}
$$

$\mathrm{U}_{\tilde{\mathrm{S}}_{1} \tilde{\mathrm{S}}_{1}}:$

$$
\begin{aligned}
U_{\tilde{S}_{1}^{\dagger} \tilde{S}_{1}} & =\tilde{\lambda}_{H 1}|H|^{2} \delta_{i j}+\frac{\partial^{2} V}{\partial \tilde{S}_{1 i}^{\dagger} \partial \tilde{S}_{1 j}}, \\
U_{\tilde{S}_{1} \tilde{S}_{1}^{\dagger}} & =\tilde{\lambda}_{H 1}|H|^{2} \delta_{i j}+\frac{\partial^{2} V}{\partial \tilde{S}_{1 i} \partial \tilde{S}_{1 j}^{\dagger}} .
\end{aligned}
$$


$\mathrm{U}_{\tilde{\mathrm{S}}_{1} \mathrm{~S}_{3}}$ :

$$
\begin{gathered}
U_{\tilde{S}_{1}^{\dagger} S_{3}}=-\lambda_{3 \tilde{1}} \delta_{i j}\left(H^{T} \cdot \epsilon \cdot \sigma^{J} \cdot H\right)+\frac{\partial^{2} V}{\partial \tilde{S}_{1 i}^{\dagger} \partial S_{3 j}^{J}}, \\
U_{\tilde{S}_{1} S_{3}^{*}}=\lambda_{3 \tilde{1}}^{*} \delta_{i j}\left(H^{\dagger} \cdot \sigma^{J} \cdot \epsilon \cdot H^{*}\right)+\frac{\partial^{2} V}{\partial \tilde{S}_{1 i} \partial S_{3 j}^{J *}} .
\end{gathered}
$$

$\mathrm{U}_{\mathrm{S}_{2} \mathrm{~S}_{2}}$ :

$$
\begin{gathered}
U_{S_{2}^{\dagger} S_{2}}=\delta_{i j} \delta_{\alpha \beta} \lambda_{H 2}|H|^{2}-\lambda_{22} \delta_{i j} H_{\alpha} H_{\beta}^{*}+\frac{\partial^{2} V}{\partial S_{2 i \alpha}^{\dagger} \partial S_{2 j \beta}} \\
U_{S_{2}^{T} S_{2}^{*}}=\delta_{i j} \delta_{\alpha \beta} \lambda_{H 2}|H|^{2}-\lambda_{22} \delta_{i j} H_{\alpha}^{*} H_{\beta}+\frac{\partial^{2} V}{\partial S_{2 i \alpha}^{T} \partial S_{2 j \beta}^{*}}
\end{gathered}
$$

$\mathrm{U}_{\mathrm{S}_{2} \tilde{\mathrm{S}}_{2}}$ :

$$
\begin{gathered}
U_{S_{2}^{\dagger} \tilde{S}_{2}}=\lambda_{2 \tilde{2}} \delta_{i j} H_{\alpha}(\epsilon \cdot H)_{\beta}^{T}+\frac{\partial^{2} V}{\partial S_{2 i \alpha}^{\dagger} \partial \tilde{S}_{2 j \beta}} \\
U_{S_{2}^{T} \tilde{S}_{2}^{*}}=\lambda_{2 \tilde{2}} \delta_{i j} H_{\alpha}^{*}\left(\epsilon \cdot H^{*}\right)_{\beta}+\frac{\partial^{2} V}{\partial S_{2 i \alpha}^{T} \partial \tilde{S}_{2 j \beta}^{*}} .
\end{gathered}
$$

$\mathrm{U}_{\widetilde{\mathrm{S}}_{2} \tilde{\mathrm{S}}_{2}}$ :

$$
\begin{aligned}
U_{\tilde{S}_{2}^{\dagger} \tilde{S}_{2}} & =\delta_{i j} \delta_{\alpha \beta} \tilde{\lambda}_{H 2}|H|^{2}-\lambda_{\tilde{2} \tilde{2}} \delta_{i j} H_{\alpha} H_{\beta}^{*}+\frac{\partial^{2} V}{\partial \tilde{S}_{2 i \alpha}^{\dagger} \partial \tilde{S}_{2 j \beta}}, \\
U_{\tilde{S}_{2}^{T} \tilde{S}_{2}} & =\frac{2}{3} \lambda_{5} \epsilon^{i j k}\left[\epsilon^{\alpha \alpha_{1}} \tilde{S}_{2 j \alpha_{1}} H_{\beta}^{*}+\tilde{S}_{2 k \alpha_{1}} \epsilon^{\alpha_{1} \beta} H_{\alpha}^{*}-\epsilon^{\alpha \beta}\left(H^{\dagger} \cdot \tilde{S}_{2 k}\right)\right]+\frac{\partial^{2} V}{\partial \tilde{S}_{2 i \alpha}^{T} \partial \tilde{S}_{2 j \beta}} \\
U_{\tilde{S}_{2}^{\dagger} \tilde{S}_{2}^{*}} & =\frac{2}{3} \lambda_{5} \epsilon^{i j k}\left[\epsilon^{\alpha \alpha_{1}} \tilde{S}_{2 k \alpha_{1}} H_{\beta}+\tilde{S}_{2 k \alpha_{1}} \epsilon^{\alpha_{1} \beta} H_{\alpha}-\epsilon^{\alpha \beta}\left(\tilde{S}_{2 k}^{\dagger} \cdot H\right)\right]+\frac{\partial^{2} V}{\partial \tilde{S}_{2 i \alpha}^{\dagger} \partial \tilde{S}_{2 j \beta}^{*}} \\
U_{\tilde{S}_{2}^{T} \tilde{S}_{2}^{*}} & =\delta_{i j} \delta_{\alpha \beta} \tilde{\lambda}_{H 2}|H|^{2}-\lambda_{\tilde{2} \tilde{2}} \delta_{i j} H_{\alpha}^{*} H_{\beta}+\frac{\partial^{2} V}{\partial \tilde{S}_{2 i \alpha}^{T} \partial \tilde{S}_{2 j \beta}^{*}}
\end{aligned}
$$

$\mathrm{U}_{\widetilde{\mathrm{S}}_{2} \mathrm{~S}_{1}}$ :

$$
\begin{aligned}
& U_{\tilde{S}_{2}^{T} S_{1}}=A_{\tilde{2} 1}^{*} H_{\alpha}^{*} \delta_{i j}+\frac{\partial^{2} V}{\partial \tilde{S}_{2 i \alpha}^{T} \partial S_{1 j}} \\
& U_{\tilde{S}_{2}^{\dagger} S_{1}^{\dagger}}=A_{\tilde{2} 1} H_{\alpha} \delta_{i j}+\frac{\partial^{2} V}{\partial \tilde{S}_{2 i \alpha}^{\dagger} \partial S_{1 j}^{\dagger}}
\end{aligned}
$$

$\mathrm{U}_{\tilde{\mathrm{S}}_{2} \mathrm{~S}_{2}}$ :

$$
\begin{gathered}
U_{\tilde{S}_{2}^{\dagger} S_{2}}=\lambda_{2 \tilde{2}} \delta_{i j} H_{\beta}^{*}\left(\epsilon \cdot H^{*}\right)_{\alpha}+\frac{\partial^{2} V}{\partial \tilde{S}_{2 i \alpha}^{*} \partial S_{2 j \beta}}, \\
U_{\tilde{S}_{2}^{T} S_{2}^{*}}=\lambda_{2 \tilde{2}} \delta_{i j} H_{\beta}(\epsilon \cdot H)_{\alpha}^{T}+\frac{\partial^{2} V}{\partial \tilde{S}_{2 i \alpha} \partial S_{2 j \beta}^{*}} .
\end{gathered}
$$


$\mathrm{U}_{\widetilde{\mathrm{S}}_{2} \mathrm{~S}_{3}}$ :

$$
\begin{gathered}
U_{\tilde{S}_{2}^{\dagger} S_{3}^{*}}=A_{23} \delta_{i j}\left(\sigma^{J} \cdot H\right)_{\alpha}+\frac{\partial^{2} V}{\partial \tilde{S}_{2 i \alpha}^{*} \partial S_{3 j}^{J *}}, \\
U_{\tilde{S}_{2}^{T} S_{3}}=A_{23}^{*} \delta_{i j}\left(\sigma^{J} \cdot H^{*}\right)_{\alpha}^{T}+\frac{\partial^{2} V}{\partial \tilde{S}_{2 i \alpha} \partial S_{3 j}^{J}} .
\end{gathered}
$$

$\mathrm{U}_{\mathrm{S}_{3} \mathrm{~S}_{3}}$ :

$$
\begin{gathered}
U_{S_{3}^{\dagger} S_{3}}=\lambda_{H 3} \delta_{i j} \delta^{I J}|H|^{2}+i \lambda_{\epsilon H 3} \epsilon^{I J K}\left(H^{\dagger} \sigma^{K} H\right) \delta_{i j}+\frac{\partial^{2} V}{\partial S_{3 i}^{I *} \partial S_{3 j}^{J}} \\
U_{S_{3}^{T} S_{3}}=\lambda_{H 3} \delta_{i j} \delta^{I J}|H|^{2}-i \lambda_{\epsilon H 3} \epsilon^{I J K}\left(H^{\dagger} \sigma^{K} H\right) \delta_{i j}+\frac{\partial^{2} V}{\partial S_{3 i}^{I} \partial S_{3 j}^{J *}}
\end{gathered}
$$

$\mathrm{U}_{\mathrm{S}_{3} \mathrm{~S}_{1}}$ :

$$
\begin{gathered}
U_{S_{3}^{\dagger} S_{1}}=\lambda_{H 13} \delta_{i j}\left(H^{\dagger} \sigma^{I} H\right)+\frac{\partial^{2} V}{\partial S_{3 i}^{I *} \partial S_{1 j}} \\
U_{S_{3}^{T} S_{1}^{*}}=\lambda_{H 13}^{*} \delta_{i j}\left(H^{\dagger} \sigma^{I} H\right)+\frac{\partial^{2} V}{\partial S_{3 i}^{I} \partial S_{1 j}^{*}}
\end{gathered}
$$

$\mathrm{U}_{\mathrm{S}_{3} \tilde{\mathrm{S}}_{1}}$ :

$$
\begin{gathered}
U_{S_{3}^{\dagger} \tilde{S}_{1}}=\lambda_{3 \tilde{1}}^{*} \delta_{i j}\left(H^{\dagger} \cdot \sigma^{I} \cdot \epsilon \cdot H^{*}\right)+\frac{\partial^{2} V}{\partial S_{3 i}^{I *} \partial \tilde{S}_{1 j}} \\
U_{S_{3}^{T} \tilde{S}_{1}^{*}}=-\lambda_{3 \tilde{1}} \delta_{i j}\left(H^{T} \cdot \epsilon \cdot \sigma^{I} \cdot H^{*}\right)+\frac{\partial^{2} V}{\partial S_{3 i}^{I} \partial \tilde{S}_{1 j}^{*}}
\end{gathered}
$$

$\mathrm{U}_{\mathrm{S}_{3} \tilde{\mathrm{S}}_{2}}:$

$$
\begin{aligned}
& U_{S_{3}^{\dagger} \tilde{S}_{2}^{*}}=-A_{\tilde{2} 3} \delta_{i j}\left(\sigma^{I} \cdot H\right)_{\beta}+\frac{\partial^{2} V}{\partial S_{3 i}^{I *} \partial \tilde{S}_{2 j \beta}^{*}} \\
& U_{S_{3}^{T} \tilde{S}_{2}}=-A_{\tilde{2} 3}^{*} \delta_{i j}\left(\sigma^{I} \cdot H^{*}\right)_{\beta}^{T}+\frac{\partial^{2} V}{\partial S_{3 i}^{I} \partial \tilde{S}_{2 j \beta}}
\end{aligned}
$$

\section{Matrix structure:}

$$
\mathbf{U}_{\mathbf{S}_{\mathbf{n}} \mathbf{S}_{\mathbf{m}}}=\left(\begin{array}{cc}
U_{S_{n}^{\dagger} S_{m}} & U_{S_{n}^{\dagger} S_{m}^{*}} \\
U_{S_{n}^{T} S_{m}} & U_{S_{n}^{T} S_{m}^{*}}
\end{array}\right),
$$

with $n, m=1, \tilde{1}, 2, \tilde{2}, 3$. All combinations make up the whole matrix structure of the heavyonly $\mathbf{U}_{\mathbf{S S}}$. Here we have listed all terms involving the Higgs field as well. There are also terms coming from the potential of all leptoquarks which are found by the general formula,

$$
\begin{aligned}
U_{S_{n}^{\dagger} S_{m}} & =\frac{\partial V}{\partial S_{n}^{\dagger} \partial S_{m}}, & U_{S_{n}^{\dagger} S_{m}^{*}} & =\frac{\partial V}{\partial S_{n}^{\dagger} \partial S_{m}^{*}}, \\
U_{S_{n}^{T} S_{m}} & =\frac{\partial V}{\partial S_{n}^{T} \partial S_{m}}, & U_{S_{n}^{T} S_{m}^{*}} & =\frac{\partial V}{\partial S_{n}^{T} \partial S_{m}^{*}} .
\end{aligned}
$$




\section{A.2 $\mathrm{X}_{S L}$}

\section{$\mathrm{U}_{\mathrm{S}_{\mathrm{n}}}$ :}

$$
\begin{aligned}
& U_{S_{1} \ell}=-\left(\lambda_{p r}^{1 \mathrm{~L}}\right) \bar{q}_{p i \alpha}^{c} \epsilon^{\alpha \beta}, \quad U_{S_{1}^{\dagger} \ell^{c}}=-\left(\lambda_{p r}^{1 \mathrm{~L}}\right)^{*} \bar{q}_{p i \alpha} \epsilon^{\alpha \beta}, \\
& U_{S_{1}^{\dagger} q}=2\left(\lambda^{\not B L}\right)^{*} \bar{q}_{p k \alpha}^{c} \epsilon^{\alpha \beta} \epsilon^{i j k}, \quad U_{S_{1}^{\dagger} q^{c}}=\left(\lambda_{p r}^{1 \mathrm{~L}}\right)^{\dagger} \delta_{i j} \bar{\ell}_{p \alpha} \epsilon^{\alpha \beta}, \\
& U_{S_{1} q}=\left(\lambda_{p r}^{1 \mathrm{~L}}\right)^{T} \delta_{i j} \bar{\ell}_{p \alpha}^{c} \epsilon^{\alpha \beta}, \quad U_{S_{1} q^{c}}=2\left(\lambda_{p r}^{\not B L}\right) \epsilon^{i j k} \bar{q}_{p k \alpha} \epsilon^{\alpha \beta} \text {, } \\
& U_{S_{1}^{\dagger} u}=\left(\lambda_{p r}^{\not B R}\right)^{*} \bar{d}_{p k}^{c} \epsilon^{i j k}, \quad U_{S_{1}^{\dagger} u^{c}}=-\left(\lambda_{p r}^{1 \mathrm{R}}\right)^{\dagger} \bar{e}_{p} \delta_{i j}, \\
& U_{S_{1} u}=-\left(\lambda_{p r}^{1 \mathrm{R}}\right)^{T} \bar{e}_{p}^{c} \delta_{i j}, \quad U_{S_{1} u^{c}}=\left(\lambda_{p r}^{\not B R}\right) \epsilon^{i j k} \bar{d}_{p k}, \\
& U_{S_{1} e}=-\left(\lambda_{p r}^{1 \mathrm{R}}\right) \bar{u}_{p i}^{c}, \quad U_{S_{1}^{\dagger} e^{c}}=-\left(\lambda_{p r}^{1 \mathrm{R}}\right)^{*} \bar{u}_{p i}, \\
& U_{S_{1}^{\dagger} d}=-\left(\lambda_{p r}^{\not B R}\right)^{\dagger} \bar{u}_{p k}^{c} \epsilon^{i j k}, \quad U_{S_{1} d^{c}}=-\left(\lambda_{p r}^{\not B R}\right)^{T} \epsilon^{i j k} \bar{u}_{p k}, \\
& U_{\tilde{S}_{1}^{\dagger} u}=-2\left(\tilde{\lambda}_{p r}^{1 \not B}\right)^{\dagger} \bar{u}_{p k}^{c} \epsilon^{i j k}, \quad U_{\tilde{S}_{1} u^{c}}=2\left(\tilde{\lambda}_{p r}^{1 \not B}\right) \bar{u}_{p k} \epsilon^{i j k}, \\
& U_{\tilde{S}_{1} e}=-\left(\tilde{\lambda}_{p r}^{1}\right) \bar{d}_{p i}^{c}, \quad U_{\tilde{S}_{1}^{\dagger} e^{c}}=-\left(\tilde{\lambda}_{p r}^{1}\right)^{*} \bar{d}_{p i}, \\
& U_{\tilde{S}_{1} d}=-\left(\tilde{\lambda}_{p r}^{1}\right)^{T} \bar{e}_{p}^{c} \delta_{i j}, \quad U_{\tilde{S}_{1}^{\dagger} d^{c}}=-\left(\tilde{\lambda}_{p r}^{1}\right)^{\dagger} \bar{e}_{p} \delta_{i j}, \\
& U_{S_{2}^{T} \ell}=-\left(\lambda_{p r}^{2 R L}\right) \bar{u}_{p i} \epsilon^{\alpha \beta}, \quad U_{S_{2}^{\dagger} \ell^{c}}=-\left(\lambda_{p r}^{2 R L}\right)^{*} \epsilon^{\alpha \beta} \bar{u}_{p i}^{c}, \\
& U_{S_{2}^{\dagger} q}=-\left(\lambda_{p r}^{2 L R}\right)^{\dagger} \delta_{\alpha \beta} \delta_{i j} \bar{e}_{p}, \quad U_{S_{2}^{T} q^{c}}=-\left(\lambda_{p r}^{2 L R}\right)^{T} \delta_{\alpha \beta} \delta_{i j} \bar{e}_{p}^{c}, \\
& U_{S_{2}^{\dagger} u}=\left(\lambda_{p r}^{2 R L}\right)^{\dagger} \delta_{i j} \bar{\ell}_{p \beta} \epsilon^{\beta \alpha}, \quad U_{S_{2}^{T} u^{c}}=\left(\lambda_{p r}^{2 R L}\right)^{T} \delta_{i j} \bar{\ell}_{p \beta}^{c} \epsilon^{\beta \alpha}, \\
& U_{S_{2}^{T} e}=-\left(\lambda_{p r}^{2 L R}\right) \bar{q}_{p i \alpha}, \quad U_{S_{2}^{\dagger} e^{c}}=-\left(\lambda_{p r}^{2 L R}\right)^{*} \bar{q}_{p i \alpha}^{c}, \\
& U_{\tilde{S}_{2}^{T} \ell}=-\left(\tilde{\lambda}_{p r}\right) \bar{d}_{p i} \epsilon^{\alpha \beta}, \quad U_{\tilde{S}_{2}^{\dagger} \ell^{c}}=-\left(\tilde{\lambda}_{p r}\right)^{*} \bar{d}_{p i}^{c} \epsilon^{\alpha \beta}, \\
& U_{\tilde{S}_{2}^{\dagger} d}=\left(\tilde{\lambda}_{p r}\right)^{\dagger} \delta_{i j} \bar{\ell}_{p \beta} \epsilon^{\beta \alpha}, \quad U_{\tilde{S}_{2}^{T} d^{c}}=\left(\tilde{\lambda}_{p r}\right)^{T} \delta_{i j} \bar{\ell}_{p \beta}^{c} \epsilon^{\beta \alpha} \text {, } \\
& U_{S_{3}^{T} \ell}=-\left(\lambda_{p r}^{3 L}\right) \bar{q}_{p i \alpha}^{c} \epsilon^{\alpha \gamma} \sigma_{\gamma \beta}^{I}, \quad U_{S_{3}^{\dagger} \ell^{c}}=\left(\lambda_{p r}^{3 L}\right)^{*} \bar{q}_{p i \alpha} \epsilon^{\gamma \alpha} \sigma_{\beta \gamma}^{I}, \\
& U_{S_{3}^{T} q}=-\left(\lambda_{p r}^{3 L}\right)^{T} \bar{\ell}_{p \alpha}^{c} \sigma_{\gamma \alpha}^{I} \epsilon^{\beta \gamma} \delta_{i j}, \quad U_{S_{3}^{\dagger} q^{c}}=\left(\lambda_{p r}^{3 L}\right)^{\dagger} \bar{\ell}_{p \alpha} \sigma_{\alpha \gamma}^{I} \epsilon^{\gamma \beta} \delta_{i j}, \\
& U_{S_{3}^{\dagger} q}=2\left(\lambda_{p r}^{3 \not B}\right)^{\dagger} \epsilon^{i j k}\left(\bar{q}_{p k}^{c} \cdot \epsilon \cdot \sigma^{I}\right)_{\beta}, \quad U_{S_{3}^{T} q^{c}}=2\left(\lambda_{p r}^{3 \not B}\right) \epsilon^{i j k}\left(\bar{q}_{p k} \cdot \sigma^{I} \cdot \epsilon\right)_{\beta} .
\end{aligned}
$$

\section{Matrix structure:}

$$
\begin{aligned}
& \mathbf{U}_{\mathbf{S}_{\mathbf{1}} \ell}=\left(\begin{array}{cc}
0 & U_{S_{1}^{\dagger} \ell^{c}} \\
U_{S_{1} \ell} & 0
\end{array}\right), \quad \mathbf{U}_{\mathbf{S}_{\mathbf{1}} \mathbf{q}}=\left(\begin{array}{cc}
U_{S_{1}^{\dagger} q} & U_{S_{1}^{\dagger} q^{c}} \\
U_{S_{1} q} & U_{S_{1} q^{c}}
\end{array}\right) \\
& \mathbf{U}_{\mathbf{S}_{\mathbf{1}} \mathbf{u}}=\left(\begin{array}{cc}
U_{S_{1}^{\dagger} u} & U_{S_{1}^{\dagger} u^{c}} \\
U_{S_{1} u} & U_{S_{1} u^{c}}
\end{array}\right), \quad \mathbf{U}_{\mathbf{S}_{\mathbf{1}} \mathbf{d}}=\left(\begin{array}{cc}
U_{S_{1}^{\dagger} d} & 0 \\
0 & U_{S_{1} d^{c}}
\end{array}\right), \quad \mathbf{U}_{\mathbf{S}_{\mathbf{1}} \mathbf{e}}=\left(\begin{array}{cc}
0 & U_{S_{1}^{\dagger} e^{c}} \\
U_{S_{1} e} & 0
\end{array}\right), \\
& \mathbf{U}_{\tilde{\mathbf{S}}_{1} \mathbf{u}}=\left(\begin{array}{cc}
U_{\tilde{S}_{1}^{\dagger} u} & 0 \\
0 & U_{\tilde{S}_{1} u^{c}}
\end{array}\right), \quad \mathbf{U}_{\tilde{\mathbf{S}}_{1} \mathbf{e}}=\left(\begin{array}{cc}
0 & U_{\tilde{S}_{1}^{\dagger} e^{c}} \\
U_{\tilde{S}_{1} e} & 0
\end{array}\right), \quad \mathbf{U}_{\tilde{\mathbf{S}}_{1} \mathbf{d}}=\left(\begin{array}{cc}
0 & U_{\tilde{S}_{1}^{\dagger} d^{c}} \\
U_{\tilde{S}_{1} d}
\end{array}\right), \\
& \mathbf{U}_{\mathbf{S}_{\mathbf{2}} \ell}=\left(\begin{array}{cc}
0 & U_{S_{2}^{\dagger} \ell^{c}} \\
U_{S_{2}^{T} \ell} & 0
\end{array}\right), \quad \mathbf{U}_{\mathbf{S}_{\mathbf{2}} \mathbf{d}}=\left(\begin{array}{cc}
U_{S_{2}^{\dagger} q} & 0 \\
0 & U_{S_{2}^{T} q^{c}}
\end{array}\right)
\end{aligned}
$$




$$
\begin{aligned}
& \mathbf{U}_{\mathbf{S}_{\mathbf{2}} \mathbf{u}}=\left(\begin{array}{cc}
U_{S_{2}^{\dagger} u} & 0 \\
0 & U_{S_{2}^{T} u^{c}}
\end{array}\right), \quad \mathbf{U}_{\mathbf{S}_{\mathbf{2}} \mathbf{e}}=\left(\begin{array}{cc}
0 & U_{S_{2}^{\dagger} e^{c}} \\
U_{S_{2}^{T} e} & 0
\end{array}\right), \\
& \mathbf{U}_{\tilde{\mathbf{S}}_{\mathbf{2}} \ell}=\left(\begin{array}{cc}
0 & U_{\tilde{S}_{2}^{\dagger} \ell^{c}} \\
U_{\tilde{S}_{2}^{T} \ell} & 0
\end{array}\right), \quad \mathbf{U}_{\tilde{\mathbf{S}}_{\mathbf{2}} \mathbf{d}}=\left(\begin{array}{cc}
U_{\tilde{S}_{2}^{\dagger} d} & 0 \\
0 & U_{\tilde{S}_{2}^{T} d^{c}}
\end{array}\right) \text {, } \\
& \mathbf{U}_{\mathbf{S}_{\mathbf{3}} \ell}=\left(\begin{array}{cc}
0 & U_{S_{3}^{\dagger} \ell^{c}} \\
U_{S_{3}^{T} \ell} & 0
\end{array}\right), \quad \mathbf{U}_{\mathbf{S}_{\mathbf{3}} \mathbf{q}}=\left(\begin{array}{cc}
U_{S_{3}^{\dagger} q} & U_{S_{3}^{\dagger} q^{c}} \\
U_{S_{3}^{T} q} & U_{S_{3}^{T} q^{c}}
\end{array}\right) .
\end{aligned}
$$

\section{$\mathrm{U}_{\mathrm{S}_{\mathrm{n}} \mathrm{H}}$ :}

$$
\begin{aligned}
& U_{S_{1}^{\dagger} H}=\lambda_{H 1} H_{\beta}^{*} S_{1 i}+A_{\tilde{2} 1} \tilde{S}_{2 i \beta}^{*}+\lambda_{H 13}^{*} S_{3 i}^{I}\left(H^{\dagger} \cdot \sigma^{I}\right)_{\beta}, \\
& U_{S_{1}^{\dagger} H^{*}}=\lambda_{H 1} H_{\beta} S_{1 i}^{\dagger}+\lambda_{H 13}^{*} S_{3 i}^{I}\left(\sigma^{I} \cdot H\right)_{\beta}^{T}, \\
& U_{S_{1} H}=\lambda_{H 1} H_{\beta}^{*} S_{1 i}+\lambda_{H 13} S_{3 i}^{I \dagger}\left(H^{\dagger} \cdot \sigma^{I}\right)_{\beta}, \\
& U_{S_{1} H^{*}}=\lambda_{H 1} H_{\beta} S_{1 i}^{\dagger}+A_{\tilde{2} 1}^{*} \tilde{S}_{2 i \beta}+\lambda_{H 13} S_{3 i}^{I^{\dagger}}\left(\sigma^{I} \cdot H\right)_{\beta}^{T}, \\
& U_{\tilde{S}_{1}^{\dagger} H}=\lambda_{H \tilde{1}} H_{\beta}^{*} \tilde{S}_{1 i}-\lambda_{3 \tilde{1}}\left[\left(H^{T} \cdot \epsilon \cdot \sigma^{I}\right)_{\beta}-\left(\epsilon \cdot \sigma^{I} \cdot H\right)_{\beta}^{T}\right] S_{3 i}^{I}, \\
& U_{\tilde{S}_{1}^{\dagger} H^{*}}=\lambda_{H \tilde{1}} H_{\beta} \tilde{S}_{1 i}, \\
& U_{\tilde{S}_{1} H}=\lambda_{H \tilde{1}} H_{\beta}^{*} \tilde{S}_{1 i}^{\dagger}, \\
& U_{\tilde{S}_{1} H^{*}}=\lambda_{H \tilde{1}} H_{\beta} \tilde{S}_{1 i}^{\dagger}-\lambda_{3 \tilde{1}}^{*} S_{3 i}^{I \dagger}\left[\left(\sigma^{I} \cdot \epsilon \cdot H^{*}\right)_{\beta}^{T}-\left(H^{\dagger} \cdot \sigma^{I} \cdot \epsilon\right)_{\beta}\right] \text {, } \\
& U_{S_{2}^{\dagger} H}=\lambda_{H 2} H_{\beta}^{*} S_{2 i \alpha}^{*}-\lambda_{2 \tilde{2}} \delta_{\alpha \beta}\left(H^{T} \cdot \epsilon \cdot \tilde{S}_{2 i}^{*}\right)-\lambda_{2 \tilde{2}} H_{\alpha}\left(\epsilon \cdot \tilde{S}_{2 i}^{*}\right)_{\beta}^{T} \\
& -\lambda_{22}\left(\epsilon \cdot S_{2 i}^{*}\right)_{\beta}^{T}\left(\epsilon \cdot H^{*}\right)_{\alpha}^{T}, \\
& U_{S_{2}^{\dagger} H^{*}}=\lambda_{H 2} H_{\beta} S_{2 i \alpha}^{*}-\lambda_{22}\left(H^{T} \cdot \epsilon \cdot S_{2 i}^{*}\right) \epsilon^{\alpha \beta}, \\
& U_{S_{2}^{T} H}=\lambda_{H 2} H_{\beta}^{*} S_{2 i \alpha}-\lambda_{22} \epsilon^{\beta \alpha}\left(S_{2 i}^{T} \cdot \epsilon \cdot H^{*}\right), \\
& U_{S_{2}^{T} H^{*}}=\lambda_{H 2} H_{\beta} S_{2 i \alpha}+\lambda_{2 \tilde{2}}^{*} \delta_{\alpha \beta}\left(\tilde{S}_{2 i} \cdot \epsilon \cdot H^{*}\right)+\lambda_{2 \tilde{2}}^{*} H_{\alpha}^{*}\left(\tilde{S}_{2 i}^{T} \cdot \epsilon\right)_{\beta} \\
& -\lambda_{22}\left(H^{T} \cdot \epsilon\right)_{\alpha}\left(S_{2 i}^{T} \cdot \epsilon\right)_{\beta} \text {, } \\
& U_{\tilde{S}_{2}^{\dagger} H}=\tilde{\lambda}_{H 2} H_{\beta}^{*} \tilde{S}_{2 i \alpha}+A_{\tilde{2} 1} S_{1 i}^{\dagger} \delta_{\alpha \beta}-A_{\tilde{2} 3} S_{3 i}^{I \dagger} \sigma_{\alpha \beta}^{I}-\lambda_{\tilde{2} \tilde{2}} \delta_{\alpha \beta}\left(H^{\dagger} \cdot \tilde{S}_{2 i}\right) \\
& +\frac{1}{3} \lambda_{5} \epsilon^{i j k}\left(-2 \epsilon^{\alpha \alpha_{1}} \tilde{S}_{2 j \alpha_{1}} \tilde{S}_{2 k \beta}+\tilde{S}_{2 k}^{T} \cdot \epsilon \cdot \tilde{S}_{2 j} \delta_{\alpha \beta}\right), \\
& U_{\tilde{S}_{2}^{\dagger} H^{*}}=\tilde{\lambda}_{H 2} H_{\beta} \tilde{S}_{2 i \alpha}+\lambda_{2 \tilde{2}}^{*} \epsilon^{\alpha \beta}\left(H^{\dagger} \cdot S_{2 i}^{*}\right)+\lambda_{2 \tilde{2}}^{*} S_{2 i \beta}^{*}\left(\epsilon \cdot H^{*}\right)_{\alpha}^{T} \\
& -\lambda_{\tilde{2} \tilde{2}} \epsilon^{\alpha \beta}\left(H^{T} \cdot \epsilon \cdot \tilde{S}_{2 i}^{*}\right) \text {, } \\
& U_{\tilde{S}_{2}^{T} H}=\tilde{\lambda}_{H 2} H_{\beta}^{*} \tilde{S}_{2 i \alpha}^{*}-\lambda_{2 \tilde{2}}\left(S_{2 i}^{T} \cdot H\right) \epsilon^{\beta \alpha}-\lambda_{2 \tilde{2}} S_{2 i \beta}\left(H^{T} \cdot \epsilon\right)_{\alpha} \\
& -\lambda_{\tilde{2} \tilde{2}} \epsilon^{\beta \alpha}\left(\tilde{S}_{2 i}^{T} \cdot \epsilon \cdot H^{*}\right), \\
& U_{\tilde{S}_{2}^{T} H^{*}}=\tilde{\lambda}_{H 2} H_{\beta} \tilde{S}_{2 i \alpha}^{*}+A_{\tilde{2} 1}^{*} \delta_{\alpha \beta} S_{1 i}-A_{\tilde{2} 3}^{*} \sigma_{\beta \alpha}^{I} S_{3 i}^{I}-\lambda_{\tilde{2} \tilde{2}}\left(\tilde{S}_{2 i}^{\dagger} \cdot H\right) \\
& +\frac{1}{3} \lambda_{5} \epsilon^{i j k}\left(-2 \epsilon^{\alpha \alpha_{1}} \tilde{S}_{2 j \alpha_{1}}^{*} \tilde{S}_{2 k \beta}^{*}+\tilde{S}_{2 k}^{\dagger} \cdot \epsilon \cdot \tilde{S}_{2 j}^{*} \delta_{\alpha \beta}\right)
\end{aligned}
$$




$$
\begin{aligned}
U_{S_{3}^{\dagger} H}= & \lambda_{H 3} H_{\beta}^{*} S_{3 i}^{I}+\lambda_{H 13}\left(H^{\dagger} \cdot \sigma^{I}\right)_{\beta} S_{1 i}-i \lambda_{\epsilon H 3} \epsilon^{I J K}\left(H^{\dagger} \cdot \sigma^{J}\right)_{\beta} S_{3 i}^{K}, \\
U_{S_{3}^{\dagger} H^{*}}= & \lambda_{H 3} H_{\beta} S_{3 i}^{I}+\lambda_{H 13}\left(\sigma^{I} \cdot H\right)_{\beta}^{T} S_{1 i}-i \lambda_{\epsilon H 3} \epsilon^{I J K}\left(\sigma^{J} \cdot H\right)_{\beta}^{T} S_{3 i}^{K} \\
& +\lambda_{3 \tilde{1}} \tilde{S}_{1 i}\left[\left(H^{\dagger} \cdot \sigma^{I} \cdot \epsilon\right)-\left(\sigma^{I} \cdot \epsilon \cdot H^{*}\right)^{T}\right]_{\beta}, \\
U_{S_{3}^{T} H}= & \lambda_{H 3} H_{\beta}^{*} S_{3 i}^{I *}+\lambda_{H 13}^{*}\left(H^{\dagger} \cdot \sigma^{I}\right)_{\beta} S_{1 i}^{\dagger}-i \lambda_{\epsilon H 3} \epsilon^{I J K}\left(H^{\dagger} \cdot \sigma^{K}\right)_{\beta} S_{3 i}^{J *} \\
& -\lambda_{3 \tilde{1}} \tilde{S}_{1 i}^{\dagger}\left[\left(H^{T} \cdot \epsilon \cdot \sigma^{I}\right)-\left(\epsilon \cdot \sigma^{I} \cdot H\right)^{T}\right]_{\beta}, \\
U_{S_{3}^{T} H^{*}}= & \lambda_{H 3} H_{\beta} S_{3 i}^{I *}+\lambda_{H 13}^{*} S_{1 i}^{\dagger}\left(\sigma^{I} \cdot H\right)_{\beta}^{T}-i \lambda_{\epsilon H 3} \epsilon^{I J K}\left(\sigma^{K} \cdot H\right)_{\beta}^{T} S_{3 i}^{J *}
\end{aligned}
$$

\section{Matrix structure:}

$$
\mathbf{U}_{\mathbf{S}_{\mathbf{n}} \mathbf{H}}=\left(\begin{array}{cc}
U_{S_{n}^{\dagger} H} & U_{S_{n}^{\dagger} H^{*}} \\
U_{S_{n}^{T} H} & U_{S_{n}^{T} H^{*}}
\end{array}\right)
$$

\section{A.3 $\mathbf{X}_{L S}$}

\section{$\mathrm{U}_{\mathrm{fS}_{\mathrm{n}}}$ :}

$$
\begin{aligned}
& U_{\bar{\ell}^{c} S_{1}}=\left(\lambda_{p r}^{1 \mathrm{~L}}\right)^{T} \epsilon^{\alpha \beta} q_{r j \beta}, \quad U_{\bar{\ell} S_{1}^{\dagger}}=\left(\lambda_{p r}^{1 \mathrm{~L}}\right)^{\dagger} \epsilon^{\alpha \beta} q_{r j \beta}^{c}, \\
& U_{\bar{q} S_{1}}=2\left(\lambda_{p r}^{\not B L}\right) \epsilon^{i j k} \epsilon^{\alpha \beta} q_{r j \beta}^{c}, \quad U_{\bar{q} S_{1}^{\dagger}}=-\left(\lambda_{p r}^{1 \mathrm{~L}}\right)^{*} \epsilon^{\alpha \beta} \ell_{r \beta}^{c} \delta_{i j}, \\
& U_{\bar{q}^{c} S_{1}}=-\left(\lambda_{p r}^{1 \mathrm{~L}}\right) \epsilon^{\alpha \beta} \ell_{r \beta} \delta_{i j}, \quad U_{\bar{q}^{c} S_{1}^{\dagger}}=2\left(\lambda_{p r}^{\not B L}\right)^{*} \epsilon^{i j k} \epsilon^{\alpha \beta} q_{r k \beta}, \\
& U_{\bar{u} S_{1}}=-\left(\lambda_{p r}^{\not B R}\right)^{T} \epsilon^{i j k} d_{r k}^{c}, \quad U_{\bar{u} S_{1}^{\dagger}}=-\left(\lambda_{p r}^{1 \mathrm{R}}\right)^{*} e_{r}^{c} \delta_{i j}, \\
& U_{\bar{u}^{c} S_{1}}=-\left(\lambda_{p r}^{1 \mathrm{R}}\right) e_{r} \delta_{i j}, \quad U_{\bar{u}^{c} S_{1}^{\dagger}}=-\left(\lambda_{p r}^{\not B R}\right)^{\dagger} \epsilon^{i j k} d_{r k}, \\
& U_{\bar{e}^{c} S_{1}}=-\left(\lambda_{p r}^{1 \mathrm{R}}\right)^{T} u_{r j}, \quad U_{\bar{e} S_{1}^{\dagger}}=-\left(\lambda_{p r}^{1 \mathrm{R}}\right)^{\dagger} u_{r j}^{c}, \\
& U_{\bar{d} S_{1}}=\left(\lambda_{p r}^{\not B R}\right) \epsilon^{i j k} u_{r k}^{c}, \quad U_{\bar{d}^{c} S_{1}^{\dagger}}=\left(\lambda_{p r}^{\not B R}\right)^{*} \epsilon_{i j k} u_{r k}, \\
& U_{\bar{u} \tilde{S}_{1}}=2\left(\tilde{\lambda}_{p r}^{1 \not B}\right) \epsilon^{i j k} u_{r k}^{c}, \quad U_{\bar{u}^{c} \tilde{S}_{1}^{\dagger}}=-2\left(\tilde{\lambda}_{p r}^{1 \not B}\right)^{\dagger} \epsilon^{i j k} u_{r k}, \\
& U_{\bar{e}^{c} \tilde{S}_{1}}=-\left(\tilde{\lambda}_{p r}^{1}\right)^{T} d_{r j}, \quad U_{\bar{e} \tilde{S}_{1}^{\dagger}}=-\left(\tilde{\lambda}_{p r}^{1}\right)^{\dagger} d_{r j}^{c}, \\
& U_{\bar{d}^{c} \tilde{S}_{1}}=-\left(\tilde{\lambda}_{p r}^{1}\right) e_{r} \delta_{i j}, \quad U_{\bar{d} \tilde{S}_{1}^{\dagger}}=-\left(\tilde{\lambda}_{p r}^{1}\right)^{*} e_{r}^{c} \delta_{i j}, \\
& U_{\bar{\ell}^{c} S_{2}}=-\left(\lambda_{p r}^{2 R L}\right)^{T} \epsilon^{\beta \alpha} u_{r j}^{c}, \quad U_{\bar{\ell}_{2}^{*}}=\left(\lambda_{p r}^{2 R L}\right)^{\dagger} \epsilon^{\alpha \beta} u_{r j}, \\
& U_{\bar{q} S_{2}}=-\left(\lambda_{p r}^{2 L R}\right) \delta_{\alpha \beta} \delta_{i j} e_{r}, \quad U_{\bar{q}^{c} S_{2}^{*}}=-\left(\lambda_{p r}^{2 L R}\right)^{*} \delta_{\alpha \beta} \delta_{i j} e_{r}^{c}, \\
& U_{\bar{u} S_{2}}=-\left(\lambda_{p r}^{2 R L}\right) \delta_{i j} \epsilon^{\beta \alpha} \ell_{r \alpha} \alpha, \quad U_{\bar{u}^{c} S_{2}^{*}}=\left(\lambda_{p r}^{2 R L}\right)^{*} \delta_{i j} \ell_{r \alpha}^{c} \epsilon^{\alpha \beta} \text {, } \\
& U_{\bar{e}^{c} S_{2}}=-\left(\lambda_{p r}^{2 L R}\right)^{T} q_{p j \beta}^{c}, \quad U_{\bar{e} S_{2}^{*}}=-\left(\lambda_{p r}^{2 L R}\right)^{\dagger} q_{r j \beta}, \\
& U_{\bar{\ell}^{c} \tilde{S}_{2}}=\left(\tilde{\lambda}_{p r}\right)^{T} d_{r i}^{c} \epsilon^{\alpha \beta}, \quad U_{\bar{\ell}_{2}^{*}}=(\tilde{\lambda} p r)^{\dagger} d_{r j} \epsilon^{\alpha \beta}, \\
& U_{\bar{d} \tilde{S}_{2}}=-\left(\tilde{\lambda}_{p r}\right) \delta_{i j} \epsilon^{\beta \alpha} \ell_{r \alpha}, \quad U_{\bar{d}^{c} \tilde{S}_{2}^{*}}=\left(\tilde{\lambda}_{p r}\right)^{*} \delta_{i j} \ell_{r \alpha}^{c} \epsilon^{\alpha \beta}, \\
& U_{\bar{\ell}^{c} S_{3}}=-\left(\lambda_{p r}^{3 L}\right)^{T}\left(q_{r j} \cdot \epsilon \cdot \sigma^{J}\right)_{\alpha}, \quad U_{\bar{\ell} S_{3}^{*}}=\left(\lambda_{p r}^{3 L}\right)^{\dagger} \sigma_{\alpha \alpha_{1}}^{J} \epsilon^{\alpha_{1} \alpha_{2}} q_{r j \alpha_{2}}^{c}, \\
& U_{\bar{q}^{c} S_{3}}=-\left(\lambda_{p r}^{3 L}\right) \delta_{i j} \epsilon^{\alpha \alpha_{1}} \sigma_{\alpha_{1} \alpha_{2}}^{I} \ell_{r \alpha_{2}}, \quad U_{\bar{q} S_{3}^{*}}=\left(\lambda_{p r}^{3 L}\right)^{*}\left(\ell_{r}^{c} \cdot \sigma^{J} \cdot \epsilon\right)_{\alpha},
\end{aligned}
$$




$$
U_{\bar{q} S_{3}}=2\left(\lambda_{p r}^{3 \not B}\right) \epsilon^{i j k} \sigma_{\alpha \alpha_{1}}^{J} \epsilon^{\alpha_{1} \alpha_{2}} q_{r k \alpha_{2}}^{c}, \quad U_{\bar{q}^{c} S_{3}^{*}}=2\left(\lambda_{p r}^{3 \not B}\right)^{\dagger} \epsilon^{i j k} \epsilon^{\alpha \alpha_{1}} \sigma_{\alpha_{1} \alpha_{2}}^{J} q_{r k \alpha_{2}} .
$$

\section{Matrix structure:}

$$
\begin{aligned}
& \mathbf{U}_{\ell \mathbf{S}_{\mathbf{1}}}=\left(\begin{array}{cc}
0 & U_{\bar{\ell} S_{1}^{\dagger}} \\
U_{\bar{\ell}^{c} S_{1}} & 0
\end{array}\right), \quad \mathbf{U}_{\mathbf{q} \mathbf{S}_{\mathbf{1}}}=\left(\begin{array}{cc}
U_{\bar{q} S_{1}} & U_{\bar{q} S_{1}^{\dagger}} \\
U_{\bar{q}^{c} S_{1}} & U_{\bar{q}^{c} S_{1}^{\dagger}}^{\dagger}
\end{array}\right) \\
& \mathbf{U}_{\mathbf{u S}}=\left(\begin{array}{cc}
U_{\bar{u} S_{1}} & U_{\bar{u} S_{1}^{\dagger}} \\
U_{\bar{u}^{c} S_{1}} & U_{\bar{u}^{c} S_{1}^{\dagger}}^{\dagger}
\end{array}\right), \quad \mathbf{U}_{\mathbf{d} \mathbf{S}_{\mathbf{1}}}=\left(\begin{array}{cc}
U_{\bar{d} S_{1}} & 0 \\
0 & U_{\bar{d}^{c} S_{1}^{\dagger}}^{\dagger}
\end{array}\right), \quad \mathbf{U}_{\mathbf{e} \mathbf{S}_{\mathbf{1}}}=\left(\begin{array}{cc}
0 & U_{\bar{e} S_{1}^{\dagger}} \\
U_{\bar{e}^{c} S_{1}} & 0
\end{array}\right), \\
& \mathbf{U}_{\mathbf{u} \tilde{\mathbf{S}}_{1}}=\left(\begin{array}{cc}
U_{\bar{u} \tilde{S}_{1}} & 0 \\
0 & U_{\bar{\ell}^{c} \tilde{S}_{1}^{\dagger}}^{\dagger}
\end{array}\right), \quad \mathbf{U}_{\mathbf{e} \tilde{\mathbf{S}}_{\mathbf{1}}}=\left(\begin{array}{cc}
0 & U_{\bar{e} \tilde{S}_{1}^{\dagger}} \\
U_{\bar{e}^{c} \tilde{S}_{1}} & 0
\end{array}\right), \quad \mathbf{U}_{\mathbf{d} \tilde{\mathbf{S}}_{1}}=\left(\begin{array}{cc}
0 & U_{\bar{d} \tilde{S}_{1}^{\dagger}} \\
U_{\bar{d}^{c} \tilde{S}_{1}} & 0
\end{array}\right), \\
& \mathbf{U}_{\ell \mathbf{S}_{\mathbf{2}}}=\left(\begin{array}{cc}
0 & U_{\bar{\ell} S_{2}^{*}} \\
U_{\bar{\ell}^{c} S_{2}} & 0
\end{array}\right), \quad \mathbf{U}_{\mathbf{q} \mathbf{S}_{\mathbf{2}}}=\left(\begin{array}{cc}
U_{\bar{q} S_{2}} & 0 \\
0 & U_{\bar{q}^{c} S_{2}^{*}}
\end{array}\right) \text {, } \\
& \mathbf{U}_{\mathbf{u S}_{\mathbf{2}}}=\left(\begin{array}{cc}
U_{\bar{u} S_{2}} & 0 \\
0 & U_{\bar{u}^{c} S_{2}^{*}}^{*}
\end{array}\right), \quad \mathbf{U}_{\mathbf{e S}}=\left(\begin{array}{cc}
0 & U_{\bar{e}} S_{2}^{*} \\
U_{\bar{e}^{c} S_{2}} & 0
\end{array}\right), \\
& \mathbf{U}_{\ell \tilde{\mathbf{S}}_{\mathbf{2}}}=\left(\begin{array}{cc}
0 & U_{\bar{\ell} \tilde{S}_{2}^{*}} \\
U_{\bar{\ell}^{c} \tilde{S}_{2}} & 0
\end{array}\right), \quad \mathbf{U}_{\mathbf{d} \tilde{\mathbf{S}}_{\mathbf{2}}}=\left(\begin{array}{cc}
U_{\bar{d} \tilde{S}_{2}} & 0 \\
0 & U_{\bar{d}^{c} \tilde{S}_{2}^{*}}^{*}
\end{array}\right), \\
& \mathbf{U}_{\ell \mathbf{S}_{3}}=\left(\begin{array}{cc}
0 & U_{\bar{\ell} S_{3}^{*}} \\
U_{\bar{\ell}^{c} S_{3}} & 0
\end{array}\right), \quad \mathbf{U}_{\mathbf{q} \mathbf{S}_{3}}=\left(\begin{array}{cc}
U_{\bar{q} S_{3}} & U_{\bar{q} S_{3}^{*}} \\
U_{\bar{q}^{c} S_{3}} & U_{\bar{q}^{c} S_{3}^{*}}
\end{array}\right) \text {. }
\end{aligned}
$$

\section{$\mathrm{U}_{\mathrm{HS}_{\mathrm{n}}}$ :}

$$
\begin{aligned}
U_{H^{\dagger} S_{1}}= & \lambda_{H 1} H_{\alpha} S_{1 j}^{\dagger}+A_{\tilde{2} 1}^{*} \tilde{S}_{2 j \alpha}+\lambda_{H 13} S_{3 j}^{J \dagger}\left(\sigma^{J} \cdot H\right)_{\alpha}^{T}, \\
U_{H^{\dagger} S_{1}^{\dagger}}= & \lambda_{H 1} H_{\alpha} S_{1 j}+\lambda_{H 13}^{*} S_{3 j}^{J}\left(\sigma^{J} \cdot H\right)_{\alpha}^{T} \\
U_{H^{T} S_{1}}= & \lambda_{H 1} H_{\alpha}^{*} S_{1 j}^{\dagger}+\lambda_{H 13} S_{3 j}^{J \dagger}\left(H^{\dagger} \cdot \sigma^{J}\right)_{\alpha} \\
U_{H^{T} S_{1}^{\dagger}}= & \lambda_{H 1} H_{\alpha}^{*} S_{1 j}+A_{21} \tilde{S}_{2 j \alpha}^{*}+\lambda_{H 13}^{*}\left(H^{\dagger} \cdot \sigma^{J}\right) S_{3 j}^{J}, \\
U_{H^{\dagger} \tilde{S}_{1}}= & \lambda_{H \tilde{1}} H_{\alpha} \tilde{S}_{1 j}^{\dagger}+\lambda_{3 \tilde{1}}^{*}\left[\left(\sigma^{I} \cdot \epsilon \cdot H^{*}\right)-\left(H^{\dagger} \cdot \sigma^{I} \cdot \epsilon\right)^{T}\right]_{\alpha} S_{3 j}^{I \dagger}, \\
U_{H^{\dagger} \tilde{S}_{1}^{\dagger}=} & \lambda_{H \tilde{1}} H_{\alpha} \tilde{S}_{1 j}, \\
U_{H^{T} \tilde{S}_{1}}= & \lambda_{H \tilde{1}} H_{\alpha}^{*} \tilde{S}_{1 j}^{\dagger}, \\
U_{H^{T} \tilde{S}_{1}^{\dagger}=} & \lambda_{H \tilde{1}} H_{\alpha}^{*} \tilde{S}_{1 j}-\lambda_{3 \tilde{1}}\left[\left(H^{T} \cdot \epsilon \cdot \sigma^{I}\right)-\left(\epsilon \cdot \sigma^{I} \cdot H\right)^{T}\right]_{\alpha} S_{3 j}^{I}, \\
U_{H^{\dagger} S_{2}}= & \lambda_{H 2} H_{\alpha} S_{2 j \beta}-\lambda_{2 \tilde{2}}^{*}\left[H_{\beta}^{*}\left(\tilde{S}_{2 j}^{T} \cdot \epsilon\right)_{\alpha}-\delta_{\alpha \beta}\left(\tilde{S}_{2 j}^{T} \cdot \epsilon \cdot H^{*}\right)\right] \\
& -\lambda_{22}\left(S_{2 j} \cdot \epsilon\right)_{\alpha}^{T}\left(H^{T} \cdot \epsilon\right)_{\beta}^{T}, \\
U_{H^{\dagger} S_{2}^{*}}= & \lambda_{H 2} H_{\alpha} S_{2 j \beta}^{*}-\lambda_{22} \epsilon^{\beta \alpha}\left(H^{T} \cdot \epsilon \cdot S_{2 j}^{*}\right), \\
U_{H^{T} S_{2}}= & \lambda_{H 2} H_{\alpha}^{*} S_{2 j \beta}-\lambda_{22} \epsilon^{\alpha \beta}\left(S_{2 j}^{T} \cdot \epsilon \cdot H^{*}\right), \\
U_{H^{T} S_{2}^{*}}= & \lambda_{H 2} H_{\alpha} S_{2 j \beta}^{*}-\lambda_{2 \tilde{2}}\left[H_{\beta}\left(\epsilon \cdot \tilde{S}_{2 i}^{*}\right)_{\alpha}+\left(H^{T} \cdot \epsilon \cdot \tilde{S}_{2 j}^{*}\right) \delta_{\alpha \beta}\right] \\
& -\lambda_{22}\left(\epsilon \cdot S_{2 j}^{*}\right)_{\alpha}\left(\epsilon \cdot H^{*}\right)_{\beta},
\end{aligned}
$$




$$
\begin{aligned}
U_{H^{\dagger} \tilde{S}_{2}}= & \tilde{\lambda}_{H 2} H_{\alpha} \tilde{S}_{2 j \beta}^{*}+A_{\tilde{2} 1}^{*} \delta_{\alpha \beta} S_{1 j}-A_{\tilde{2} 3}^{*} S_{3 j}^{I} \sigma_{\alpha \beta}^{I}-\lambda_{2 \tilde{2}}\left(\tilde{S}_{2 i}^{\dagger} \cdot H\right) \\
& +\frac{1}{3} \lambda_{5} \epsilon^{i j k}\left[-2 \tilde{S}_{2 i \alpha 1} \epsilon^{\alpha \beta} \tilde{S}_{2 k \alpha}+\left(\tilde{S}_{2 i} \cdot \epsilon \cdot \tilde{S}_{2 k}\right) \delta_{\alpha \beta}\right] \\
U_{H^{\dagger} \tilde{S}_{2}^{*}}= & \tilde{\lambda}_{H 2} H_{\alpha} \tilde{S}_{2 j \beta}+\lambda_{2 \tilde{2}}^{*}\left[S_{2 j \alpha}^{*}\left(\epsilon \cdot H^{*}\right)_{\beta}-\epsilon^{\alpha \beta}\left(H^{\dagger} \cdot S_{2 j}^{*}\right)\right] \\
& +\lambda_{\tilde{2} \tilde{2}} \epsilon^{\alpha \beta}\left(H^{T} \cdot \epsilon \cdot \tilde{S}_{2 j}^{*}\right) \\
U_{H^{T} \tilde{S}_{2}}= & \tilde{\lambda}_{H 2} H_{\alpha}^{*} \tilde{S}_{2 j \beta}^{*}-\lambda_{2 \tilde{2}}\left[\epsilon^{\alpha \beta}\left(S_{2 j}^{T} \cdot H\right)-S_{2 j \alpha}\left(H^{T} \cdot \epsilon\right)_{\beta}^{T}\right] \\
& -\lambda_{\tilde{2} \tilde{2}} \epsilon^{\alpha \beta}\left(\tilde{S}_{2 j} \cdot \epsilon \cdot H^{*}\right) \\
U_{H^{T} \tilde{S}_{2}^{*}}= & \tilde{\lambda}_{H 2} H_{\alpha}^{*} \tilde{S}_{2 i \beta}+A_{\tilde{2} 1} S_{1 j}^{\dagger} \delta_{\alpha \beta}-A_{\tilde{2} 3} S_{3 j}^{I \dagger} \sigma_{\beta \alpha}^{I}-\lambda_{\tilde{2} \tilde{2}}\left(H^{\dagger} \cdot \tilde{S}_{2 i}\right) \\
& +\frac{1}{3} \lambda_{5} \epsilon^{i j k}\left[-2 \tilde{S}_{2 i \alpha 1}^{*} \epsilon^{\alpha} \beta \tilde{S}_{2 k \alpha}^{*}+\left(\tilde{S}_{2 i}^{\dagger} \cdot \epsilon \cdot \tilde{S}_{2 k}^{*}\right) \delta_{\alpha \beta}\right] \\
U_{H^{\dagger} S_{3}}= & \lambda_{H 3} H_{\alpha} S_{3 j}^{J \dagger}+\lambda_{H 13}^{*}\left(\sigma^{J} \cdot H\right)_{\alpha} S_{1 j}^{\dagger}-i \lambda_{\epsilon H 3} \epsilon^{I J K}\left(H^{\dagger} \cdot \sigma^{I}\right)_{\alpha}^{T} S_{3 j}^{K \dagger}, \\
U_{H^{\dagger} S_{3}^{*}}= & \lambda_{H 3} H_{\alpha} S_{3 j}^{J}+\lambda_{H 13}\left(\sigma^{J} \cdot H\right)_{\alpha} S_{1 j}+i \lambda_{\epsilon H 3} \epsilon^{I J K}\left(\sigma^{I} \cdot H\right)_{\alpha} S_{3 j}^{K} \\
& +\lambda_{3 \tilde{1}}^{*} \tilde{S}_{1 j}\left[\left(\sigma^{J} \cdot \epsilon \cdot H^{*}\right)-\left(H^{\dagger} \cdot \sigma^{J} \cdot \epsilon\right)^{T}\right]_{\alpha}, \\
U_{H^{T} S_{3}}= & \lambda_{H 3} H_{\alpha}^{*} S_{3 j}^{J \dagger}+\lambda_{H 13}^{*}\left(\sigma^{I} \cdot H^{*}\right)_{\alpha} S_{1 j}^{\dagger}-i \lambda_{\epsilon H 3} \epsilon^{I J K}\left(H^{\dagger} \cdot \sigma^{I}\right)_{\alpha}^{T} S_{3 j}^{K \dagger} \\
& -\lambda_{3 \tilde{1}} \tilde{S}_{1 j}^{\dagger}\left[\left(\epsilon \cdot \sigma^{J} \cdot H\right)-\left(H^{T} \cdot \epsilon \cdot \sigma^{J}\right)^{T}\right]_{\alpha}, \\
U_{H^{T} S_{3}^{*}}= & \lambda_{H 3} H_{\alpha}^{*} S_{3 j}^{J}+\lambda_{H 13}\left(H^{\dagger} \cdot \sigma^{J}\right)_{\alpha}^{T} S_{1 j}+i \lambda_{\epsilon H 3} \epsilon^{I J K}\left(H^{\dagger} \cdot \sigma^{I}\right)_{\alpha} S_{3 j}^{K} .
\end{aligned}
$$

\section{Matrix structure:}

$$
\mathbf{U}_{\mathbf{H S}_{\mathbf{n}}}=\left(\begin{array}{cc}
U_{H^{\dagger} S_{n}} & U_{H^{\dagger} S_{n}^{*}} \\
U_{H^{T} S_{n}} & U_{H^{T} S_{n}^{*}}^{*}
\end{array}\right) .
$$

\section{A.4 $\mathbf{X}_{L L}$}

$\mathrm{U}_{\mathrm{ff}}$ :

$$
\begin{aligned}
& U_{\bar{\ell}^{c}}=\left(\lambda_{p r}^{1 \mathrm{~L}}\right)^{\dagger} S_{1 j}^{\dagger} \epsilon^{\alpha \beta} \\
& U_{\bar{\ell}^{c} q}=-\left(\lambda_{p r}^{1 \mathrm{~L}}\right)^{T} S_{1 j} \epsilon^{\alpha \beta} \\
& +\left(\lambda_{p r}^{3 L}\right)^{\dagger} S_{3 j}^{J} \sigma_{\alpha \gamma}^{J} \epsilon^{\gamma \beta}, \\
& +\left(\lambda_{p r}^{3 L}\right)^{T} S_{3 j}^{J} \epsilon^{\beta \gamma} \sigma_{\gamma \alpha}^{J}, \\
& U_{\bar{\ell} u}=\left(\lambda_{p r}^{2 R L}\right)^{\dagger}\left(\epsilon \cdot S_{2 i}^{*}\right)_{\alpha} \text {, } \\
& U_{\bar{\ell}^{c} u^{c}}=\left(\lambda_{p r}^{2 R L}\right)^{T}\left(\epsilon \cdot S_{2 j}\right)_{\alpha}^{T} \text {, } \\
& U_{\bar{\ell} e}=\left(y_{E}\right)_{p r} H_{\alpha}, \\
& U_{\bar{\ell}^{c} e^{c}}=\left(y_{E}\right)_{p r}^{*} H_{\alpha}^{*} \text {, } \\
& U_{\bar{\ell} d}=\left(\tilde{\lambda}_{p r}\right)^{\dagger} \epsilon^{\alpha \beta} \tilde{S}_{2 i \beta}^{*}, \\
& U_{\bar{\ell}^{c} d^{c}}=\left(\tilde{\lambda}_{p r}\right)^{T} \epsilon^{\alpha \beta} \tilde{S}_{2 j \beta} \text {, } \\
& U_{\bar{q} \ell^{c}}=-\left(\lambda_{p r}^{1 \mathrm{~L}}\right)^{*} S_{1 i}^{\dagger} \epsilon^{\alpha \beta}+\left(\lambda_{p r}^{3 L}\right)^{*} S_{3 i}^{I} \sigma_{\beta \gamma}^{I} \epsilon^{\gamma \alpha}, \\
& U_{\bar{q}^{c} \ell}=-\left(\lambda_{p r}^{1 \mathrm{~L}}\right) \epsilon^{\alpha \beta} S_{1 i}-\left(\lambda_{p r}^{3 L}\right) S_{3 i}^{I} \sigma_{\alpha \gamma}^{I} \epsilon^{\gamma \beta}, \\
& U_{\bar{q} q^{c}}=-2\left(\lambda_{p r}^{\not B L}\right) \epsilon^{\alpha \beta} \epsilon^{i j k} S_{1 k}, \\
& U_{\bar{q}^{c} \bar{q}}=-2\left(\lambda_{p r}^{\not B L}\right)^{*} \epsilon^{\alpha \beta} \epsilon^{i j k} S_{1 k}^{\dagger} \text {, } \\
& -2\left(\lambda_{p r}^{3 \not B}\right) \epsilon^{i j k} S_{3 k}^{K} \sigma_{\alpha \gamma}^{K} \epsilon^{\gamma \beta}, \\
& -\left(\lambda_{p r}^{3 \not B}\right)^{\dagger} \epsilon^{i j k} \epsilon^{\alpha \gamma} \sigma_{\gamma \beta}^{K} S_{3 k}^{K \dagger} \\
& U_{\bar{q} u}=\left(y_{U}\right)_{p r} \delta_{i j} \epsilon^{\alpha \beta} H_{\beta}^{*} \text {, } \\
& U_{\bar{q}^{c} u^{c}}=\left(y_{U}\right)_{p r}^{*} \delta_{i j} \epsilon^{\alpha \beta} H_{\beta} \text {, } \\
& U_{\bar{q} e}=-\left(\lambda_{p r}^{2 L R}\right) S_{2 i \alpha}, \\
& U_{\bar{q}^{c} e^{c}}=-\left(\lambda_{p r}^{2 L R}\right)^{*} S_{2 i \alpha}^{*}, \\
& U_{\bar{q} d}=\left(y_{D}\right)_{p r} H_{\alpha} \delta_{i j} \text {, } \\
& U_{\bar{q}^{c} d^{c}}=\left(y_{D}\right)_{p r}^{*} \delta_{i j} H_{\alpha}^{*},
\end{aligned}
$$




$$
\begin{aligned}
U_{\bar{u} \ell} & =-\left(\lambda_{p r}^{2 R L}\right) S_{2 i \gamma} \epsilon^{\gamma \beta}, & U_{\bar{u}^{c} \ell^{c}} & =\left(\lambda_{p r}^{2 R L}\right)^{*} \epsilon^{\beta \gamma} S_{2 i \gamma}^{*}, \\
U_{\bar{u} q} & =-\left(y_{U}\right)_{p r}^{\dagger} \epsilon^{\alpha \beta} H_{\alpha} \delta_{i j}, & U_{\bar{u}^{c} \bar{q}^{c}} & =-\left(y_{U}\right)_{p r}^{T} \epsilon^{\beta \alpha} H_{\alpha}^{*} \delta_{i j}, \\
U_{\bar{u} u^{c}} & =-2\left(\tilde{\lambda}_{p r}^{1 \not B}\right) \epsilon^{i j k} \tilde{S}_{1 k}, & U_{\bar{u}^{c} u} & =2\left(\tilde{\lambda}_{p r}^{1 \not B}\right)^{\dagger} \epsilon^{i j k} \tilde{S}_{1 k}^{\dagger}, \\
U_{\bar{u} e^{c}} & =-\left(\lambda_{p r}^{1 R}\right)^{*} S_{1 i}^{\dagger}, & U_{\bar{u}^{c} e} & =-\left(\lambda_{p r}^{1 R}\right) S_{1 i}, \\
U_{\bar{u} d^{c}} & =-\left(\lambda_{p r}^{\not B R}\right)^{T} \epsilon^{i j k} S_{1 k}, & U_{\bar{u}^{c} d} & =-\left(\lambda_{p r}^{\not B R}\right)^{\dagger} \epsilon^{i j k} S_{1 k}^{\dagger}, \\
U_{\bar{e} \ell} & =\left(y_{E}\right)_{p r}^{\dagger} H_{\beta}^{*}, & U_{\bar{e}^{c} \ell^{c}} & =\left(y_{E}\right)_{p r}^{T} H_{\beta}, \\
U_{\bar{e} q} & =-\left(\lambda_{p r}^{2 L R}\right)^{\dagger} S_{2 j \alpha}^{\dagger}, & U_{\bar{e}^{c} q^{c}} & =-\left(\lambda_{p r}^{2 L R}\right)^{T} S_{2 j \beta}, \\
U_{\bar{e} u^{c}} & =\left(\lambda_{p r}^{1 \mathrm{R}}\right)^{\dagger} S_{1 j}^{\dagger}, & U_{\bar{e}^{c} u} & =-\left(\lambda_{p r}^{1 \mathrm{R}}\right)^{T} S_{1 j}, \\
U_{\bar{e} d^{c}} & =-\left(\tilde{\lambda}_{p r}\right)^{\dagger} \tilde{S}_{1 j}, & U_{\bar{e}^{c} d} & =-\left(\tilde{\lambda}_{p r}\right)^{T} \tilde{S}_{1 j}, \\
U_{\bar{d} \ell} & =-\left(\tilde{\lambda}_{p r}\right) \tilde{S}_{2 i \alpha} \epsilon^{\alpha \beta}, & U_{\bar{d}^{c} \ell^{c}} & =\left(\tilde{\lambda}_{p r}\right)^{*} \epsilon^{\beta \alpha} \tilde{S}_{2 i \alpha}^{*}, \\
U_{\bar{d} q} & =\left(y_{D}\right)_{p r}^{\dagger} H_{\beta}^{*} \delta_{i j}, & U_{\bar{d}^{c} q^{c}} & =\left(y_{D}\right)_{p r}^{T} H_{\alpha} \delta_{i j}, \\
U_{\bar{d} u^{c}} & =\left(\lambda_{p r}^{\not B R} \epsilon^{i j k} S_{1 k},\right. & U_{\bar{d}^{c} u} & =\left(\lambda_{p r}^{\not B R}\right)^{*} \epsilon^{i j k} S_{1 k}^{\dagger}, \\
U_{\bar{d} e^{c}} & =-\left(\tilde{\lambda}_{p r}\right)^{*} \tilde{S}_{1 i}^{\dagger}, & U_{\bar{d}^{c} e} & =-\left(\tilde{\lambda}_{p r}\right) \tilde{S}_{1 i} .
\end{aligned}
$$

\section{Matrix structure:}

$$
\begin{aligned}
& \mathbf{U}_{\ell \mathbf{q}}=\left(\begin{array}{cc}
0 & U_{\bar{\ell} q^{c}} \\
U_{\bar{\ell}^{c} q} & 0
\end{array}\right), \quad \mathbf{U}_{\ell \mathbf{u}}=\left(\begin{array}{cc}
U_{\bar{\ell} u} & 0 \\
0 & U_{\bar{\ell}^{c} u^{c}}
\end{array}\right), \\
& \mathbf{U}_{\ell \mathbf{e}}=\left(\begin{array}{cc}
U_{\bar{\ell} e} & 0 \\
0 & U_{\bar{\ell}^{c} e^{c}}
\end{array}\right), \quad \mathbf{U}_{\ell \mathbf{d}}=\left(\begin{array}{cc}
U_{\bar{\ell} d} & 0 \\
0 & U_{\bar{\ell}^{c} d^{c}}
\end{array}\right), \\
& \mathbf{U}_{\mathbf{q} \ell}=\left(\begin{array}{cc}
0 & U_{\bar{q} \ell^{c}} \\
U_{\bar{q}^{c} \ell} & 0
\end{array}\right), \quad \mathbf{U}_{\mathbf{q q}}=\left(\begin{array}{cc}
0 & U_{\bar{q} q^{c}} \\
U_{\bar{q}^{c} q} & 0
\end{array}\right), \quad \mathbf{U}_{\mathbf{q u}}=\left(\begin{array}{cc}
U_{\bar{q} u} & 0 \\
0 & U_{\bar{q}^{c} u^{c}}
\end{array}\right) \\
& \mathbf{U}_{\mathbf{q e}}=\left(\begin{array}{cc}
U_{\bar{q} e} & 0 \\
0 & U_{\bar{q}^{c} e^{c}}
\end{array}\right), \quad \mathbf{U}_{\mathbf{q d}}=\left(\begin{array}{cc}
U_{\bar{q} d} & 0 \\
0 & U_{\bar{q}^{c} d^{c}}
\end{array}\right) \\
& \mathbf{U}_{\mathbf{u} \ell}=\left(\begin{array}{cc}
U_{\bar{u} \ell} & 0 \\
0 & U_{\bar{u}^{c} \ell^{c}}
\end{array}\right), \quad \mathbf{U}_{\mathbf{u q}}=\left(\begin{array}{cc}
U_{\bar{u} q} & 0 \\
0 & U_{\bar{u}^{c} q^{c}}
\end{array}\right), \quad \mathbf{U}_{\mathbf{u u}}=\left(\begin{array}{cc}
0 & U_{\bar{u} u^{c}} \\
U_{\bar{u}^{c} u} & 0
\end{array}\right), \\
& \mathbf{U}_{\mathbf{u e}}=\left(\begin{array}{cc}
0 & U_{\bar{u} e^{c}} \\
U_{\bar{u}^{c} e} & 0
\end{array}\right), \quad \mathbf{U}_{\mathbf{u d}}=\left(\begin{array}{cc}
0 & U_{\bar{u} d^{c}} \\
U_{\bar{u}^{c} d} & 0
\end{array}\right) \text {, } \\
& \mathbf{U}_{\mathbf{d} \ell}=\left(\begin{array}{cc}
U_{\bar{d} \ell} & 0 \\
0 & U_{\bar{d}^{c} \ell^{c}}
\end{array}\right), \quad \mathbf{U}_{\mathbf{d q}}=\left(\begin{array}{cc}
U_{\bar{d} q} & 0 \\
0 & U_{\bar{d}^{c}} q^{c}
\end{array}\right) \\
& \mathbf{U}_{\mathbf{d u}}=\left(\begin{array}{cc}
0 & U_{\bar{d} u^{c}} \\
U_{\bar{d}^{c} u} & 0
\end{array}\right), \quad \mathbf{U}_{\mathbf{d e}}=\left(\begin{array}{cc}
0 & U_{\bar{d} e^{c}} \\
U_{\bar{d}^{c} e} & 0
\end{array}\right) \\
& \mathbf{U}_{\mathbf{e} \ell}=\left(\begin{array}{cc}
U_{\bar{e} \ell} & 0 \\
0 & U_{\bar{e}^{c} \ell c}^{c}
\end{array}\right), \quad \mathbf{U}_{\mathbf{e q}}=\left(\begin{array}{cc}
U_{\bar{e} q} & 0 \\
0 & U_{\bar{e}^{c} q^{c}}
\end{array}\right), \\
& \mathbf{U}_{\mathbf{e u}}=\left(\begin{array}{cc}
0 & U_{\bar{e} u^{c}} \\
U_{\bar{e}^{c} u} & 0
\end{array}\right), \quad \mathbf{U}_{\mathbf{e d}}=\left(\begin{array}{cc}
0 & U_{\bar{e} d^{c}} \\
U_{\bar{e}^{c} d} & 0
\end{array}\right) \\
& \mathbf{U}_{\ell \mathbf{u}}=\mathbf{U}_{\ell \ell}=\mathbf{U}_{\mathbf{q e}}=\mathbf{U}_{\mathbf{u} \ell}=\mathbf{U}_{\mathbf{u u}}=\mathbf{U}_{\mathbf{d d}}=\mathbf{U}_{\mathbf{d e}}=\mathbf{U}_{\mathbf{e e}}=\mathbf{U}_{\mathbf{e q}}=\mathbf{U}_{\mathbf{e d}}=\mathbf{0}
\end{aligned}
$$


$\mathrm{U}_{\mathrm{Hf}}$ :

$$
\begin{aligned}
U_{H^{\dagger} \ell} & =\left(y_{E}\right)_{p r}^{\dagger} \bar{e}_{p} \delta_{\alpha \beta}, & U_{H^{T} \ell^{c}} & =\left(y_{E}\right)_{p r}^{T} \bar{e}_{p}^{c} \delta_{\alpha \beta}, \\
U_{H^{\dagger} q} & =\left(y_{D}\right)_{p r}^{\dagger} \bar{d}_{p j} \delta_{\alpha \beta}, & U_{H^{\dagger} q^{c}} & =\left(y_{U}\right)_{p r}^{T} \bar{u}_{p j}^{c} \epsilon^{\beta \alpha}, \\
U_{H^{T} q} & =\left(y_{U}\right)_{p r}^{\dagger} \epsilon^{\alpha \beta} \bar{u}_{p j}, & U_{H^{T} q^{c}} & =\left(y_{D}\right)_{p r}^{T} \bar{d}_{p j}^{c} \delta_{\alpha \beta}, \\
U_{H^{\dagger} u} & =\left(y_{U}\right)_{p r} \bar{q}_{p j \beta} \epsilon^{\beta \alpha}, & U_{H^{T} u^{c}} & =\left(y_{U}\right)_{p r}^{*} \bar{q}_{p j \beta}^{c} \epsilon^{\alpha \beta}, \\
U_{H^{T} e} & =\left(y_{E}\right)_{p r} \bar{\ell}_{p \alpha}, & U_{H^{\dagger} e^{c}} & =\left(y_{E}\right)_{p r}^{*} \bar{\ell}_{p \alpha}^{c}, \\
U_{H^{T} d} & =\left(y_{D}\right)_{p r} \bar{q}_{p j \alpha}, & U_{H^{\dagger} d^{c}} & =\left(y_{D}\right)_{p r}^{*} \bar{q}_{p j \alpha}^{c} .
\end{aligned}
$$

Matrix structure:

$$
\begin{aligned}
& \mathbf{U}_{\mathbf{H} \ell}=\left(\begin{array}{cc}
U_{H^{\dagger} \ell} & 0 \\
0 & U_{H^{T} \ell^{c}}
\end{array}\right), \mathbf{U}_{\mathbf{H q}}=\left(\begin{array}{cc}
U_{H^{\dagger} q} & U_{H^{\dagger} q^{c}} \\
U_{H^{T} q} & U_{H^{T} q^{c}}
\end{array}\right), \\
& \mathbf{U}_{\mathbf{H u}}=\left(\begin{array}{cc}
U_{H^{\dagger} u} & 0 \\
0 & U_{H^{T} u^{c}}
\end{array}\right), \quad \mathbf{U}_{\mathbf{H d}}=\left(\begin{array}{cc}
0 & U_{H^{\dagger} d^{c}} \\
U_{H^{T} d} & 0
\end{array}\right), \quad \mathbf{U}_{\mathbf{H e}}=\left(\begin{array}{cc}
0 & U_{H^{\dagger} e^{c}} \\
U_{H^{T} e} & 0
\end{array}\right) .
\end{aligned}
$$

$\mathrm{U}_{\mathrm{fH}}$ :

$$
\begin{aligned}
U_{\bar{\ell} H} & =\left(y_{E}\right)_{p r} e_{r} \delta_{\alpha \beta}, & U_{\bar{\ell}^{c} H^{*}} & =\left(y_{E}\right)_{p r}^{*} e_{r}^{c} \delta_{\alpha \beta}, \\
U_{\bar{q} H} & =\left(y_{D}\right)_{p r} d_{r i} \delta_{\alpha \beta}, & U_{\bar{q} H^{*}} & =\left(y_{U}\right)_{p r} u_{r i} \epsilon^{\alpha \beta}, \\
U_{\bar{q}^{c} H} & =\left(y_{U}\right)_{p r}^{*} \epsilon^{\alpha \beta} u_{r i}^{c}, & U_{\bar{q}^{c} H^{*}} & =\left(y_{D}\right)_{p r}^{*} d_{r i}^{c} \delta_{\alpha \beta}, \\
U_{\bar{u} H} & =\left(y_{U}\right)_{p r}^{\dagger} \epsilon^{\beta \alpha} q_{r i \alpha}, & U_{\bar{u}^{c} H^{*}} & =\left(y_{U}\right)_{p r}^{T} \epsilon^{\alpha \beta} q_{r i \alpha}^{c}, \\
U_{\bar{d}^{c} H} & =\left(y_{D}\right)_{p r}^{T} q_{r i \beta}^{c}, & U_{\bar{d} H^{*}} & =\left(y_{D}\right)_{p r}^{\dagger} q_{r i \beta}, \\
U_{\bar{e}^{c} H} & =\left(y_{E}\right)_{p r}^{T} \ell_{r \beta}^{c}, & U_{\bar{e} H^{*}} & =\left(y_{E}\right)_{p r}^{\dagger} \ell_{r \beta} .
\end{aligned}
$$

Matrix structure:

$$
\begin{aligned}
& \mathbf{U}_{\ell \mathbf{H}}=\left(\begin{array}{cc}
U_{\bar{\ell} H} & 0 \\
0 & U_{\bar{\ell}^{c} H^{*}}
\end{array}\right), \quad \mathbf{U}_{\mathbf{q H}}=\left(\begin{array}{cc}
U_{\bar{q} H} & U_{\bar{q} H^{*}} \\
U_{\bar{q}^{c} H} & U_{\bar{q}^{c} H^{*}}
\end{array}\right), \\
& \mathbf{U}_{\mathbf{u H}}=\left(\begin{array}{cc}
U_{\bar{u} H} & 0 \\
0 & U_{\bar{u}^{c} H^{*}}
\end{array}\right), \quad \mathbf{U}_{\mathbf{d H}}=\left(\begin{array}{cc}
0 & U_{\bar{d} H^{*}} \\
U_{\bar{d}^{c} H} & 0
\end{array}\right), \quad \mathbf{U}_{\mathbf{e H}}=\left(\begin{array}{cc}
0 & U_{\bar{e} H^{*}} \\
U_{\bar{e}^{c} H} & 0
\end{array}\right) .
\end{aligned}
$$

$\mathrm{U}_{\mathrm{Vf}}$ :

$$
\begin{aligned}
U_{B \ell} & =-\bar{\ell}_{r \beta} g^{\prime} Y_{\ell} \gamma^{\mu}, & U_{B \ell^{c}} & =\bar{\ell}_{r \beta}^{c} g^{\prime} Y_{\ell} \gamma^{\mu}, \\
U_{W \ell} & =-\frac{g}{2} \bar{\ell}_{r \alpha_{1}} \sigma_{\alpha_{1} \beta}^{I} \gamma^{\mu}, & U_{W \ell^{c}} & =\frac{g}{2} \bar{\ell}_{r \alpha_{1}} \sigma_{\beta \alpha_{1}}^{I} \gamma^{\mu}, \\
U_{B q} & =-\bar{q}_{r j \beta} g^{\prime} Y_{q} \gamma^{\mu}, & U_{B q^{c}} & =\bar{q}_{r j \beta}^{c} g^{\prime} Y_{q} \gamma^{\mu}, \\
U_{W q} & =-\frac{g}{2} \bar{q}_{r j \alpha_{1}} \sigma_{\alpha_{1} \beta}^{I} \gamma^{\mu}, & U_{W q^{c}} & =\frac{g}{2} \bar{\ell}_{r j \alpha_{1}} \sigma_{\beta \alpha_{1}}^{I} \gamma^{\mu}, \\
U_{G q} & =-g_{s} \bar{q}_{r i \beta} T_{i j}^{A} \gamma^{\mu}, & U_{G q^{c}} & =g_{s} \bar{q}_{r i \beta}^{c} T_{j i}^{A} \gamma^{\mu}, \\
U_{B u} & =-\bar{u}_{r j} g^{\prime} Y_{u} \gamma^{\mu}, & U_{B u^{c}} & =\bar{u}_{r i}^{c} g^{\prime} Y_{u} \gamma^{\mu}, \\
U_{G u} & =-g_{s} \bar{u}_{r i} T_{i j}^{A} \gamma^{\mu}, & U_{G u^{c}} & =g_{s} \bar{u}_{r i} T_{j i}^{A} \gamma^{\mu}, \\
U_{B d} & =-\bar{d}_{r j} g^{\prime} Y_{d} \gamma^{\mu}, & U_{B d^{c}} & =\bar{d}_{r j}^{c} g^{\prime} Y_{d} \gamma^{\mu}, \\
U_{G d} & =-g_{s} \bar{d}_{r i} T_{i j}^{A} \gamma^{\mu}, & U_{G d^{c}} & =g_{s} \bar{d}_{r i}^{c} T_{j i}^{A} \gamma^{\mu}, \\
U_{B e} & =-\bar{e}_{r} g^{\prime} Y_{e} \gamma^{\mu}, & U_{B e^{c}} & =\bar{e}_{r}^{c} g^{\prime} Y_{e} \gamma^{\mu} .
\end{aligned}
$$




\section{Matrix structure:}

$$
\begin{aligned}
& \mathbf{U}_{\mathbf{V} \ell}=\left(\begin{array}{cc}
U_{B \ell} & U_{B \ell^{c}} \\
U_{W \ell} & U_{W \ell^{c}} \\
0 & 0
\end{array}\right), \quad \mathbf{U}_{\mathbf{V q}}=\left(\begin{array}{cc}
U_{B q} & U_{B q^{c}} \\
U_{W q} & U_{W q^{c}} \\
U_{G q} & U_{G q^{c}}
\end{array}\right) \\
& \mathbf{U}_{\mathbf{V u}}=\left(\begin{array}{cc}
U_{B u} & U_{B u^{c}} \\
0 & 0 \\
U_{G u} & U_{G u^{c}}
\end{array}\right), \quad \mathbf{U}_{\mathbf{V d}}=\left(\begin{array}{cc}
U_{B d} & U_{B d^{c}} \\
0 & 0 \\
U_{G d} & U_{G d^{c}}
\end{array}\right), \quad \mathbf{U}_{\mathbf{V e}}=\left(\begin{array}{cc}
U_{B e} & U_{B e^{c}} \\
0 & 0 \\
0 & 0
\end{array}\right) .
\end{aligned}
$$

$\mathrm{U}_{\mathrm{fV}}$ :

$$
\begin{aligned}
U_{\bar{\ell} B} & =-g^{\prime} Y_{\ell} \gamma^{\nu} \ell_{p \alpha}, & U_{\bar{\ell}^{c} B} & =g^{\prime} Y_{\ell} \gamma^{\nu} \ell_{p \alpha}^{c}, \\
U_{\bar{\ell} W} & =-\frac{g}{2} \sigma_{\alpha \alpha_{1}}^{I} \gamma^{\nu} \ell_{p \alpha_{1}}, & U_{\bar{\ell}^{c} W} & =\frac{g}{2} \sigma_{\alpha_{1} \alpha}^{I} \gamma^{\nu} \ell_{p \alpha_{1}}^{c}, \\
U_{\bar{q} B} & =-g^{\prime} Y_{q} \gamma^{\nu} q_{p i \alpha}, & U_{\bar{q}^{c} B} & =g^{\prime} Y_{q} \gamma^{\nu} q_{p i \alpha}^{c}, \\
U_{\bar{q} W} & =-\frac{g}{2} \sigma_{\alpha \alpha_{1}}^{I} \gamma^{\nu} q_{p i \alpha_{1}}, & U_{\bar{q}^{c} W} & =\frac{g}{2} \sigma_{\alpha_{1} \alpha}^{I} \gamma^{\nu} q_{p i \alpha_{1}}, \\
U_{\bar{q} G} & =-g_{s} \gamma^{\mu} T_{i j}^{B} q_{p j \alpha}, & U_{\bar{q}^{c} G} & =g_{s} \gamma^{\mu} T_{j i}^{B} q_{p j \alpha}^{c}, \\
U_{\bar{u} B} & =-g^{\prime} Y_{u} \gamma^{\nu} u_{p i}, & U_{\bar{u}^{c} B} & =g^{\prime} Y_{q} \gamma^{\nu} u_{p i}^{c}, \\
U_{\bar{u} G} & =-g_{s} \gamma^{\mu} T_{i j}^{B} u_{p j}, & U_{\bar{u}^{c} G} & =g_{s} \gamma^{\mu} T_{j i}^{B} q_{p j}^{c}, \\
U_{\bar{d} B} & =-g^{\prime} Y_{d} \gamma^{\nu} d_{p i}, & U_{\bar{d}^{c} B} & =g^{\prime} Y_{d} \gamma^{\nu} d_{p i}^{c}, \\
U_{\bar{d} G} & =-g_{s} \gamma^{\mu} T_{i j}^{B} d_{p j}, & U_{\bar{d}^{c} G} & =g_{s} \gamma^{\mu} T_{j i}^{B} d_{p j}^{c}, \\
U_{\bar{e} B} & =-g^{\prime} Y_{e} \gamma^{\nu} e_{p}, & U_{\bar{e}^{c} B} & =g^{\prime} Y_{e} \gamma^{\nu} e_{p} .
\end{aligned}
$$

\section{Matrix structure:}

$$
\begin{aligned}
& \mathbf{U}_{\ell \mathbf{V}}=\left(\begin{array}{ccc}
U_{\bar{\ell} B} & U_{\bar{\ell} W} & 0 \\
U_{\bar{\ell}^{c} B} & U_{\bar{\ell}^{c} W} & 0
\end{array}\right), \quad \mathbf{U}_{\mathbf{q} \mathbf{V}}=\left(\begin{array}{ccc}
U_{\bar{q} B} & U_{\bar{q} W} & U_{\bar{q} G} \\
U_{\bar{q}^{c} B} & U_{\bar{q}^{c} W} & U_{\bar{q}^{c} G}
\end{array}\right), \\
& \mathbf{U}_{\mathbf{u V}}=\left(\begin{array}{ccc}
U_{\bar{u} B} & 0 & U_{\bar{u} G} \\
U_{\bar{u} B} & 0 & U_{\bar{u}^{c} G}
\end{array}\right), \quad \mathbf{U}_{\mathbf{d V}}=\left(\begin{array}{ccc}
U_{\bar{d} B} & 0 & U_{\bar{d} G} \\
U_{\bar{d}^{c} B} & 0 & U_{\bar{d}^{c} G}
\end{array}\right), \quad \mathbf{U}_{\mathbf{e V}}=\left(\begin{array}{ccc}
U_{\bar{e} B} & 0 & 0 \\
U_{\bar{e}^{c} B} & 0 & 0
\end{array}\right) .
\end{aligned}
$$

$\mathrm{Z}_{\mathrm{S}_{\mathrm{n}} \mathrm{V}}$ :

$$
\begin{array}{ll}
Z_{S_{(1, \tilde{1})} B}^{\rho \nu}=-g^{\rho \nu} g^{\prime} Y_{S_{(1, \tilde{1})}} S_{(1, \tilde{1}) i}^{\dagger}, & \bar{Z}_{B S_{(1, \tilde{1})}}^{\mu \kappa}=-g^{\mu \kappa} g^{\prime} Y_{S_{(1, \tilde{1})}} S_{(1, \tilde{1}) j}^{\dagger}, \\
Z_{S_{(1, \tilde{1})}^{\rho \nu} B}^{\dagger}=-g^{\rho \nu} g^{\prime} Y_{S_{(1, \tilde{1})}} S_{(1, \tilde{1}) i}, & \bar{Z}_{B S_{(1, \tilde{1})}^{\mu \kappa}}^{\dagger}=-g^{\mu \kappa} g^{\prime} Y_{S_{(1, \tilde{1})}} S_{(1, \tilde{1}) j}, \\
Z_{S_{(1, \tilde{1})}}^{\rho \nu}=-g^{\rho \nu} g_{s} S_{(1, \tilde{1}) k}^{\dagger} T_{i k}^{B}, & \bar{Z}_{G S_{(1, \tilde{1})}}^{\mu \kappa}=-g^{\mu \kappa} g_{s} S_{(1, \tilde{1}) k}^{\dagger} T_{k j}^{B}, \\
Z_{S_{(1, \tilde{1})}^{\rho \nu} G}^{\nu}=-g^{\rho \nu} g_{s} T_{i k}^{B} S_{(1, \tilde{1}) k}, & \bar{Z}_{G S_{(1, \tilde{1})}^{\mu \kappa}}^{\dagger}=-g^{\mu \kappa} g_{s} T_{k j}^{B} S_{(1, \tilde{1}) k}, \\
Z_{S_{(2, \tilde{2})}^{\rho \nu} B}^{\rho \nu}=-g^{\rho \nu} g^{\prime} Y_{S_{(2, \tilde{2})}} S_{(2, \tilde{2}) i \alpha}^{*}, & \bar{Z}_{B S_{(2, \tilde{2})}^{\mu \kappa}}^{\mu \kappa}=-g^{\mu \kappa} g^{\prime} Y_{S_{(2, \tilde{2})}} S_{(2, \tilde{2}) j \beta}, \\
Z_{S_{(2, \tilde{2})}^{\rho \nu} B}^{\dagger \nu}=-g^{\rho \nu} g^{\prime} Y_{S_{(2, \tilde{2})}} S_{(2, \tilde{2}) i \alpha}, & \bar{Z}_{B S_{(2, \tilde{2})}^{\mu \kappa}}^{\dagger}=-g^{\mu \kappa} g^{\prime} Y_{S_{(2, \tilde{2})}} S_{(2, \tilde{2}) j \beta}^{*}, \\
Z_{S_{(2, \tilde{2})}^{T} W}^{\rho \nu}=-g^{\rho \nu} \frac{g}{2} \sigma_{\alpha \alpha_{1}}^{J} S_{(2, \tilde{2}) i \alpha_{1}}^{*}, & \bar{Z}_{W S_{(2, \tilde{2})}^{T}}^{\mu \kappa}=-g^{\mu \kappa} \frac{g}{2} \sigma_{\alpha_{1} \beta}^{I} S_{(2, \tilde{2}) j \alpha_{1}},
\end{array}
$$




$$
\begin{aligned}
Z_{S_{(2, \tilde{2})}^{\dagger} W}^{\rho \nu} & =-g^{\rho \nu} \frac{g}{2} \sigma_{\alpha \alpha_{1}}^{J} S_{(2, \tilde{2}) i \alpha_{1}}, & \bar{Z}_{W S_{(2, \tilde{2})}^{\dagger \kappa}}^{\mu \kappa} & =-g^{\mu \kappa} \frac{g}{2} \sigma_{\alpha_{1} \beta}^{I} S_{(2, \tilde{2}) j \alpha_{1}}^{*}, \\
Z_{S_{(2, \tilde{2})}^{\rho \nu} G} & =-g^{\rho \nu} g_{s} T_{i k}^{B} S_{(2, \tilde{2}) k \alpha}^{*}, & \bar{Z}_{G S_{(2, \tilde{2})}^{\mu \kappa}}^{T} & =-g^{\mu \kappa} g_{s} T_{k j}^{B} S_{(2, \tilde{2}) k \beta}, \\
Z_{S_{(2, \tilde{2})}^{\mu \nu} G}^{\dagger} & =-g^{\mu \nu} g_{s} T_{i k}^{B} S_{(2, \tilde{2}) k \alpha}, & \bar{Z}_{G S_{(2, \tilde{)})}^{\mu \kappa}}^{\dagger} & =-g^{\mu \kappa} g_{s} T_{k j}^{B} S_{(2, \tilde{2}) k \beta}^{*}, \\
Z_{S_{3}^{T} B}^{\rho \nu} & =-g^{\rho \nu} g^{\prime} Y_{S_{3}} S_{3 i}^{I *}, & \bar{Z}_{B S_{3}^{T}}^{\mu \kappa} & =-g^{\mu \kappa} g^{\prime} Y_{S_{3}} S_{3 j}^{J}, \\
Z_{S_{3}^{\dagger} B}^{\rho \nu} & =-g^{\rho \nu} g^{\prime} Y_{S_{3}} S_{3 i}^{I}, & \bar{Z}_{B S_{3}^{\dagger}}^{\mu \kappa} & =-g^{\mu \kappa} g^{\prime} Y_{S_{3}} S_{3 j}^{J *}, \\
Z_{S_{3}^{T} W}^{\rho \nu} & =i g^{\rho \nu} g \epsilon^{L K J} S_{3 l}^{K *}, & \bar{Z}_{W S_{3}^{T}}^{\mu \kappa} & =-i g^{\mu \kappa} g \epsilon^{L K I} S_{3 l}^{K}, \\
Z_{S_{3}^{\dagger} W}^{\rho \nu} & =i g^{\rho \nu} g \epsilon^{L K J} S_{3 l}^{K}, & \bar{Z}_{W S_{3}^{\dagger}}^{\mu \kappa} & =-i g^{\mu \kappa} g \epsilon^{L K I} S_{3 j}^{K *}, \\
Z_{S_{3}^{T} G}^{\rho \nu} & =-g^{\rho \nu} g_{s} T_{i k}^{B} S_{3 k \alpha}^{*}, & \bar{Z}_{G S_{3}^{T}}^{\mu \kappa} & =-g^{\mu \kappa} g_{s} T_{k j}^{B} S_{3 k \beta}, \\
Z_{S_{3}^{\dagger} G}^{\mu \nu} & =-g^{\mu \nu} g_{s} T_{i k}^{B} S_{3 k \alpha}, & \bar{Z}_{G S_{3}^{\dagger}}^{\mu \kappa} & =-g^{\mu \kappa} g_{s} T_{k j}^{B} S_{3 k \beta}^{*} .
\end{aligned}
$$




\section{B Supertraces vs. covariant diagrams}

The construction of covariant diagrams precedes chronologically the method of Supertraces. The essential difference between these two techniques is the point at which the CDE is applied. In Supertraces one first makes superdiagrams from the distinct components of the interaction matrix $\mathbf{X}$ and then applies the CDE in the resulting Supertrace. In the Covariant diagrams approach the components of the interaction matrix are split explicitly into, heavy-only, heavy-light and light-only contributions. In the resulting trace the CDE is applied and the log-function is ultimately Taylor expanded. Finally, from the expanded formula one reads the components of the covariant diagrams in the same manner one would read of Feynman rules and the covariant diagrams are drawn. All in all Supertraces are a more compact way to present Covariant diagrams. One can also look at [23] for a more elaborate comparison between the two approaches.

In tables 4 and 5 we present the relevant covariant diagrams for the Scalar Leptoquark Action. We have made combinations of matrices, and momentum insertions following the rules for constructing covariant diagrams in [16]. We have classified them in terms of $U, P$ and $Z$ insertions. In total we count 60 covariant diagrams.

To summarize one can now clearly see the advantage of using the method of Supertraces. From 60 Covariant Diagrams we ended up with 15 Supertrace Diagrams calculated in section 2.3 .

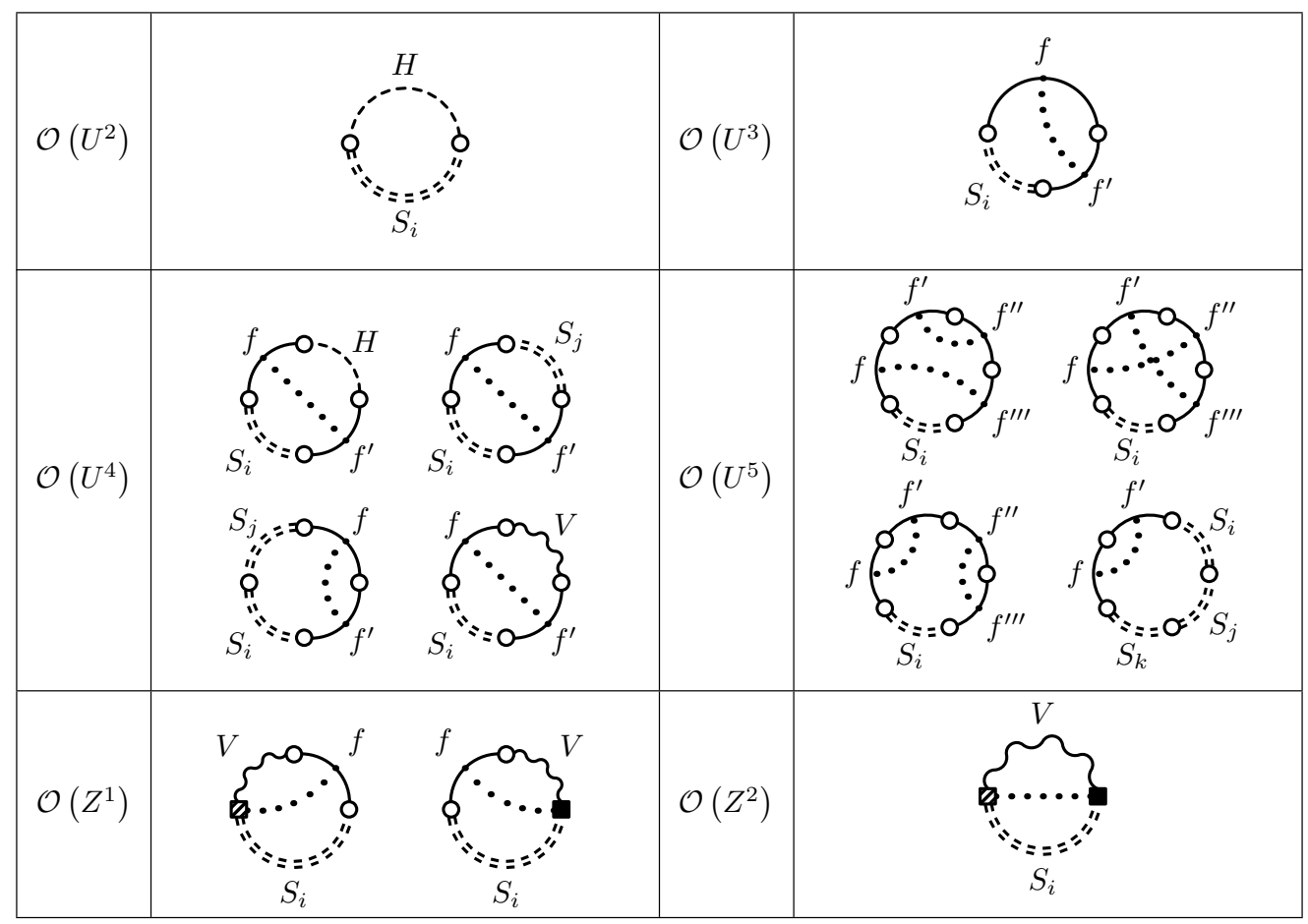

Table 4. $U$ and $Z$ only, heavy-light, covariant diagrams contributing to the construction of operators up to dimension 6 . Where $S_{i}=S_{1}, \tilde{S}_{2}, f=\ell, q, u, e, d$ and $V=B, W, G$. In total we count 13 diagrams. 


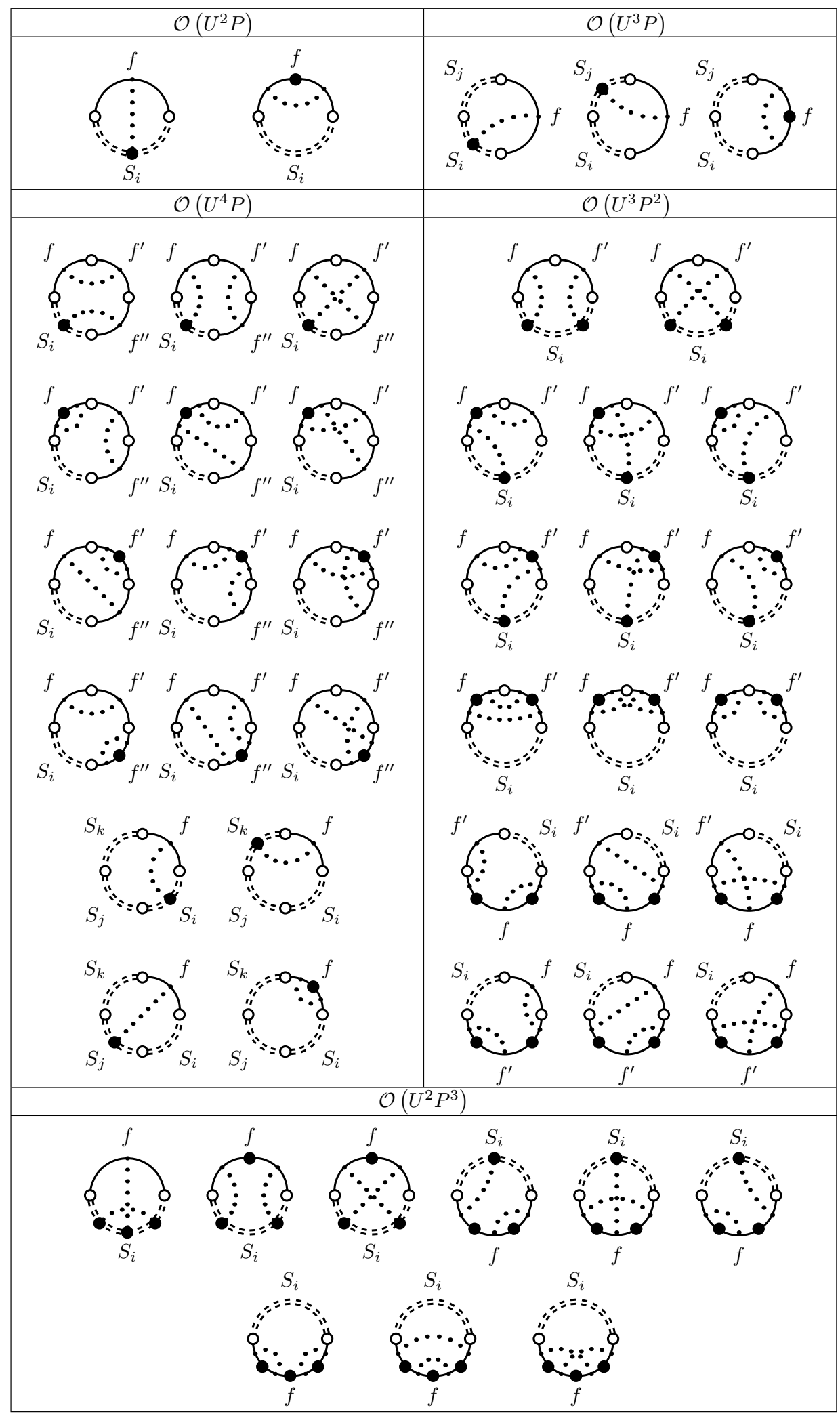

Table 5. Covariant diagrams containing powers of both $U$ and $P$ contributing to operators up to dimension 6 . In total we have 47 diagrams. 


\section{Green basis operators}

The dimension-5 operator has the same definition in both Green and Warsaw basis, as shown in table 6 .

The dimension- 6 operators in Green basis are listed in tables 7-10. Shaded ones are included in Warsaw basis too.

\begin{tabular}{|l|l|}
\hline \multicolumn{2}{|c|}{$\boldsymbol{H}^{\mathbf{2}} \boldsymbol{\psi}^{\mathbf{2}}$} \\
\hline $\mathcal{O}_{\nu \nu}$ & $\epsilon^{\alpha \beta} \epsilon^{\alpha_{1} \beta_{1}} H^{\alpha} H^{\alpha_{1}} \bar{\ell}_{p \beta}^{c} \ell_{r \beta_{1}}$ \\
\hline
\end{tabular}

Table 6. Single dimension-5 operator giving rise to neutrino masses.

\begin{tabular}{|c|c|c|c|c|c|}
\hline \multicolumn{2}{|r|}{$X^{3}$} & \multicolumn{2}{|r|}{$X^{2} H^{2}$} & \multicolumn{2}{|r|}{$H^{2} D^{4}$} \\
\hline \multirow{4}{*}{$\begin{array}{l}\mathcal{O}_{3 G} \\
\mathcal{O}_{\widetilde{3 G}} \\
\mathcal{O}_{3 W} \\
\mathcal{O}_{\widetilde{3 W}}\end{array}$} & \multirow{4}{*}{$\begin{array}{c}f^{A B C} G_{\mu}^{A \nu} G_{\nu}^{B \rho} G_{\rho}^{C \mu} \\
f^{A B C} \widetilde{G}_{\mu}^{A \nu} G_{\nu}^{B \rho} G_{\rho}^{C \mu} \\
\epsilon^{I J K} W_{\mu}^{I \nu} W_{\nu}^{J \rho} W_{\rho}^{K \mu} \\
\epsilon^{I J K} \widetilde{W}_{\mu}^{I \nu} W_{\nu}^{J \rho} W_{\rho}^{K \mu}\end{array}$} & \multirow{2}{*}{$\begin{array}{l}\mathcal{O}_{H G} \\
\mathcal{O}_{H \widetilde{G}}\end{array}$} & \multirow{2}{*}{$\begin{array}{l}G_{\mu \nu}^{A} G^{A \mu \nu}\left(H^{\dagger} H\right) \\
\widetilde{G}_{\mu \nu}^{A} G^{A \mu \nu}\left(H^{\dagger} H\right)\end{array}$} & \multicolumn{2}{|r|}{$\left(D_{\mu} D^{\mu} H\right)^{\dagger}\left(D_{\nu} D^{\nu} H\right)$} \\
\hline & & & & \multicolumn{2}{|r|}{$H^{4} D^{2}$} \\
\hline & & $\mathcal{O}_{H W}$ & $W_{\mu \nu}^{I} W^{I \mu \nu}\left(H^{\dagger} H\right)$ & $\mathcal{O}_{H \square}$ & $\left(H^{\dagger} H\right) \square\left(H^{\dagger} H\right)$ \\
\hline & & $\mathcal{O}_{H \widetilde{W}}$ & $\widetilde{W}_{\mu \nu}^{I} W^{I \mu \nu}\left(H^{\dagger} H\right)$ & $\mathcal{O}_{H D}$ & $\left(H^{\dagger} D^{\mu} H\right)^{\dagger}\left(H^{\dagger} D_{\mu} H\right)$ \\
\hline \multicolumn{2}{|r|}{$X^{2} D^{2}$} & $\mathcal{O}_{H B}$ & $B_{\mu \nu} B^{\mu \nu}\left(H^{\dagger} H\right)$ & \multirow{2}{*}{$\begin{array}{l}\mathcal{O}_{H D}^{\prime} \\
\mathcal{O}_{H D}^{\prime \prime}\end{array}$} & \multirow{2}{*}{$\begin{array}{l}\left(H^{\dagger} H\right)\left(D_{\mu} H\right)^{\dagger}\left(D^{\mu} H\right) \\
\left(H^{\dagger} H\right) D_{\mu}\left(H^{\dagger} i \overleftrightarrow{D}^{\mu} H\right)\end{array}$} \\
\hline \multirow{5}{*}{$\begin{array}{l}\mathcal{O}_{2 G} \\
\mathcal{O}_{2 W} \\
\mathcal{O}_{2 B}\end{array}$} & \multirow{5}{*}{$\begin{array}{c}-\frac{1}{2}\left(D_{\mu} G^{A \mu \nu}\right)\left(D^{\rho} G_{\rho \nu}^{A}\right) \\
-\frac{1}{2}\left(D_{\mu} W^{I \mu \nu}\right)\left(D^{\rho} W_{\rho \nu}^{I}\right) \\
-\frac{1}{2}\left(\partial_{\mu} B^{\mu \nu}\right)\left(\partial^{\rho} B_{\rho \nu}\right)\end{array}$} & $\mathcal{O}_{H \widetilde{B}}$ & $\widetilde{B}_{\mu \nu} B^{\mu \nu}\left(H^{\dagger} H\right)$ & & \\
\hline & & $\mathcal{O}_{H W B}$ & $W_{\mu \nu}^{I} B^{\mu \nu}\left(H^{\dagger} \sigma^{I} H\right)$ & \multicolumn{2}{|r|}{$H^{6}$} \\
\hline & & $\mathcal{O}_{H \widetilde{W} B}$ & $\widetilde{W}_{\mu \nu}^{I} B^{\mu \nu}\left(H^{\dagger} \sigma^{I} H\right)$ & \multirow[t]{3}{*}{$\mathcal{O}_{H}$} & \multirow[t]{3}{*}{$\left(H^{\dagger} H\right)^{3}$} \\
\hline & & \multicolumn{2}{|r|}{$H^{2} X D^{2}$} & & \\
\hline & & $\begin{array}{l}\mathcal{O}_{W D H} \\
\mathcal{O}_{B D H}\end{array}$ & $\begin{array}{c}D_{\nu} W^{I \mu \nu}\left(H^{\dagger} i \overleftrightarrow{D}_{\mu}^{I} H\right) \\
\partial_{\nu} B^{\mu \nu}\left(H^{\dagger} i \overleftrightarrow{D}_{\mu} H\right)\end{array}$ & & \\
\hline
\end{tabular}

Table 7. Bosonic operators in the Green's basis. 


\begin{tabular}{|c|c|c|c|c|c|}
\hline \multicolumn{2}{|r|}{$\psi^{2} D^{3}$} & \multicolumn{2}{|r|}{$\psi^{2} X D$} & \multicolumn{2}{|r|}{$\psi^{2} D H^{2}$} \\
\hline $\mathcal{O}_{q D}$ & $\frac{i}{2} \bar{q}\left\{D_{\mu} D^{\mu}, \not D\right\} q$ & $\mathcal{O}_{G q}$ & $\left(\bar{q} T^{A} \gamma^{\mu} q\right) D^{\nu} G_{\mu \nu}^{A}$ & $\mathcal{O}_{H q}^{(1)}$ & $\left(\bar{q} \gamma^{\mu} q\right)\left(H^{\dagger} i \overleftrightarrow{D}_{\mu} H\right)$ \\
\hline $\mathcal{O}_{u D}$ & $\frac{i}{2} \bar{u}\left\{D_{\mu} D^{\mu}, \not D\right\} u$ & $\mathcal{O}_{G q}^{\prime}$ & $\frac{1}{2}\left(\bar{q} T^{A} \gamma^{\mu} i \overleftrightarrow{D}^{\nu} q\right) G_{\mu \nu}^{A}$ & $\mathcal{O}_{H q}^{\prime(1)}$ & $(\bar{q} i \overleftrightarrow{I D} q)\left(H^{\dagger} H\right)$ \\
\hline $\mathcal{O}_{d D}$ & $\frac{i}{2} \bar{d}\left\{D_{\mu} D^{\mu}, \not D\right\} d$ & $\mathcal{O}_{\widetilde{G} q}^{\prime}$ & $\frac{1}{2}\left(\bar{q} T^{A} \gamma^{\mu} i \overleftrightarrow{D}^{\nu} q\right) \widetilde{G}_{\mu \nu}^{A}$ & $\mathcal{O}_{H q}^{\prime \prime(1)}$ & $\left(\bar{q} \gamma^{\mu} q\right) \partial_{\mu}\left(H^{\dagger} H\right)$ \\
\hline $\mathcal{O}_{\ell D}$ & $\frac{i}{2} \bar{\ell}\left\{D_{\mu} D^{\mu}, \not D\right\} \ell$ & $\mathcal{O}_{W q}$ & $\left(\bar{q} \sigma^{I} \gamma^{\mu} q\right) D^{\nu} W_{\mu \nu}^{I}$ & $\mathcal{O}_{H q}^{(3)}$ & $\left(\bar{q} \sigma^{I} \gamma^{\mu} q\right)\left(H^{\dagger} i \overleftrightarrow{D}_{\mu}^{I} H\right)$ \\
\hline $\mathcal{O}_{e D}$ & $\frac{i}{2} \bar{e}\left\{D_{\mu} D^{\mu}, \not D\right\} e$ & $\mathcal{O}_{W q}^{\prime}$ & $\frac{1}{2}\left(\bar{q} \sigma^{I} \gamma^{\mu} i \overleftrightarrow{D}^{\nu} q\right) W_{\mu \nu}^{I}$ & $\mathcal{O}_{H q}^{\prime(3)}$ & $\left(\bar{q} i \overleftrightarrow{\not D}^{I} q\right)\left(H^{\dagger} \sigma^{I} H\right)$ \\
\hline \multicolumn{2}{|c|}{$\psi^{2} H D^{2}+$ h.c. } & $\mathcal{O}_{\widetilde{W} q}^{\prime}$ & $\frac{1}{2}\left(\bar{q} \sigma^{I} \gamma^{\mu} i \overleftrightarrow{D}^{\nu} q\right) \widetilde{W}_{\mu \nu}^{I}$ & $\mathcal{O}_{H q}^{\prime \prime(3)}$ & $\left(\bar{q} \sigma^{I} \gamma^{\mu} q\right) D_{\mu}\left(H^{\dagger} \sigma^{I} H\right)$ \\
\hline $\mathcal{O}_{u H D 1}$ & $(\bar{q} u) D_{\mu} D^{\mu} \widetilde{H}$ & $\mathcal{O}_{B q}$ & $\left(\bar{q} \gamma^{\mu} q\right) \partial^{\nu} B_{\mu \nu}$ & $\mathcal{O}_{H u}$ & $\left(\bar{u} \gamma^{\mu} u\right)\left(H^{\dagger} i \overleftrightarrow{D}{ }_{\mu} H\right)$ \\
\hline $\mathcal{O}_{u H D 2}$ & $\left(\bar{q} i \sigma_{\mu \nu} D^{\mu} u\right) D^{\nu} \widetilde{H}$ & $\mathcal{O}_{B q}^{\prime}$ & $\frac{1}{2}\left(\bar{q} \gamma^{\mu} i \overleftrightarrow{D}^{\nu} q\right) B_{\mu \nu}$ & $\mathcal{O}_{H u}^{\prime}$ & $(\bar{u} i \overleftrightarrow{\not D} u)\left(H^{\dagger} H\right)$ \\
\hline $\mathcal{O}_{u H D 3}$ & $\left(\bar{q} D_{\mu} D^{\mu} u\right) \widetilde{H}$ & $\mathcal{O}_{\widetilde{B} q}^{\prime}$ & $\frac{1}{2}\left(\bar{q} \gamma^{\mu} i \overleftrightarrow{D}^{\nu} q\right) \widetilde{B}_{\mu \nu}$ & $\mathcal{O}_{H u}^{\prime \prime}$ & $\left(\bar{u} \gamma^{\mu} u\right) \partial_{\mu}\left(H^{\dagger} H\right)$ \\
\hline $\mathcal{O}_{u H D 4}$ & $\left(\bar{q} D_{\mu} u\right) D^{\mu} \widetilde{H}$ & $\mathcal{O}_{G u}$ & $\left(\bar{u} T^{A} \gamma^{\mu} u\right) D^{\nu} G_{\mu \nu}^{A}$ & $\mathcal{O}_{H d}$ & $\left(\bar{d} \gamma^{\mu} d\right)\left(H^{\dagger} i \overleftrightarrow{D}_{\mu} H\right)$ \\
\hline $\mathcal{O}_{d H D 1}$ & $(\bar{q} d) D_{\mu} D^{\mu} H$ & $\mathcal{O}_{G u}^{\prime}$ & $\frac{1}{2}\left(\bar{u} T^{A} \gamma^{\mu} i \overleftrightarrow{D}^{\nu} u\right) G_{\mu \nu}^{A}$ & $\mathcal{O}_{H d}^{\prime}$ & $(\bar{d} i \overleftrightarrow{\not D} d)\left(H^{\dagger} H\right)$ \\
\hline $\mathcal{O}_{d H D 2}$ & $\left(\bar{q} i \sigma_{\mu \nu} D^{\mu} d\right) D^{\nu} H$ & $\mathcal{O}_{\widetilde{G} u}^{\prime}$ & $\frac{1}{2}\left(\bar{u} T^{A} \gamma^{\mu} i \overleftrightarrow{D}^{\nu} u\right) \widetilde{G}_{\mu \nu}^{A}$ & $\mathcal{O}_{H d}^{\prime \prime}$ & $\left(\bar{d} \gamma^{\mu} d\right) \partial_{\mu}\left(H^{\dagger} H\right)$ \\
\hline $\mathcal{O}_{d H D 3}$ & $\left(\bar{q} D_{\mu} D^{\mu} d\right) H$ & $\mathcal{O}_{B u}$ & $\left(\bar{u} \gamma^{\mu} u\right) \partial^{\nu} B_{\mu \nu}$ & $\mathcal{O}_{H u d}$ & $\left(\bar{u} \gamma^{\mu} d\right)\left(\widetilde{H}^{\dagger} i D_{\mu} H\right)$ \\
\hline $\mathcal{O}_{d H D 4}$ & $\left(\bar{q} D_{\mu} d\right) D^{\mu} H$ & $\mathcal{O}_{B u}^{\prime}$ & $\frac{1}{2}\left(\bar{u} \gamma^{\mu} i \overleftrightarrow{D}^{\nu} u\right) B_{\mu \nu}$ & $\mathcal{O}_{H \ell}^{(1)}$ & $\left(\bar{\ell} \gamma^{\mu} \ell\right)\left(H^{\dagger} i \overleftrightarrow{D}_{\mu} H\right)$ \\
\hline $\mathcal{O}_{e H D 1}$ & $(\bar{\ell} e) D_{\mu} D^{\mu} H$ & $\mathcal{O}_{\widetilde{B} u}^{\prime}$ & $\frac{1}{2}\left(\bar{u} \gamma^{\mu} i \overleftrightarrow{D}^{\nu} u\right) \widetilde{B}_{\mu \nu}$ & $\mathcal{O}_{H \ell}^{\prime(1)}$ & $(\bar{\ell} i \overleftrightarrow{\not D} \ell)\left(H^{\dagger} H\right)$ \\
\hline $\mathcal{O}_{e H D 2}$ & $\left(\bar{\ell} i \sigma_{\mu \nu} D^{\mu} e\right) D^{\nu} H$ & $\mathcal{O}_{G d}$ & $\left(\bar{d} T^{A} \gamma^{\mu} d\right) D^{\nu} G_{\mu \nu}^{A}$ & $\mathcal{O}_{H \ell}^{\prime \prime(1)}$ & $\left(\bar{\ell} \gamma^{\mu} \ell\right) \partial_{\mu}\left(H^{\dagger} H\right)$ \\
\hline $\mathcal{O}_{e H D 3}$ & $\left(\bar{\ell} D_{\mu} D^{\mu} e\right) H$ & $\mathcal{O}_{G d}^{\prime}$ & $\frac{1}{2}\left(\bar{d} T^{A} \gamma^{\mu} i \overleftrightarrow{D}^{\nu} d\right) G_{\mu \nu}^{A}$ & $\mathcal{O}_{H \ell}^{(3)}$ & $\left(\bar{\ell} \sigma^{I} \gamma^{\mu} \ell\right)\left(H^{\dagger} i \overleftrightarrow{D}_{\mu}^{I} H\right)$ \\
\hline $\mathcal{O}_{e H D 4}$ & $\left(\bar{\ell} D_{\mu} e\right) D^{\mu} H$ & $\mathcal{O}_{\widetilde{G} d}^{\prime}$ & $\frac{1}{2}\left(\bar{d} T^{A} \gamma^{\mu} i \overleftrightarrow{D}^{\nu} d\right) \widetilde{G}_{\mu \nu}^{A}$ & $\mathcal{O}_{H \ell}^{\prime(3)}$ & $\left(\bar{\ell} i \overleftrightarrow{I^{I}} \ell \ell\right)\left(H^{\dagger} \sigma^{I} H\right)$ \\
\hline \multicolumn{2}{|c|}{$\psi^{2} X \boldsymbol{H}+$ h.c. } & $\mathcal{O}_{B d}$ & $\left(\bar{d} \gamma^{\mu} d\right) \partial^{\nu} B_{\mu \nu}$ & $\mathcal{O}_{H \ell}^{\prime \prime(3)}$ & $\left(\bar{\ell} \sigma^{I} \gamma^{\mu} \ell\right) D_{\mu}\left(H^{\dagger} \sigma^{I} H\right)$ \\
\hline $\mathcal{O}_{u G}$ & $\left(\bar{q} T^{A} \sigma^{\mu \nu} u\right) \widetilde{H} G_{\mu \nu}^{A}$ & $\mathcal{O}_{B d}^{\prime}$ & $\frac{1}{2}\left(\bar{d} \gamma^{\mu} i \overleftrightarrow{D}^{\nu} d\right) B_{\mu \nu}$ & $\mathcal{O}_{H e}$ & $\left(\bar{e} \gamma^{\mu} e\right)\left(H^{\dagger} i \overleftrightarrow{D}_{\mu} H\right)$ \\
\hline $\mathcal{O}_{u W}$ & $\left(\bar{q} \sigma^{\mu \nu} u\right) \sigma^{I} \widetilde{H} W_{\mu \nu}^{I}$ & $\mathcal{O}_{\widetilde{B} d}^{\prime}$ & $\frac{1}{2}\left(\bar{d} \gamma^{\mu} i \overleftrightarrow{D}^{\nu} d\right) \widetilde{B}_{\mu \nu}$ & $\mathcal{O}_{H e}^{\prime}$ & $(\bar{e} i \overleftrightarrow{\not D} e)\left(H^{\dagger} H\right)$ \\
\hline $\mathcal{O}_{u B}$ & $\left(\bar{q} \sigma^{\mu \nu} u\right) \widetilde{H} B_{\mu \nu}$ & $\mathcal{O}_{W \ell}$ & $\left(\bar{\ell} \sigma^{I} \gamma^{\mu} \ell\right) D^{\nu} W_{\mu \nu}^{I}$ & $\mathcal{O}_{H e}^{\prime \prime}$ & $\left(\bar{e} \gamma^{\mu} e\right) \partial_{\mu}\left(H^{\dagger} H\right)$ \\
\hline $\mathcal{O}_{d G}$ & $\left(\bar{q} T^{A} \sigma^{\mu \nu} d\right) H G_{\mu \nu}^{A}$ & $\mathcal{O}_{W \ell}^{\prime}$ & $\frac{1}{2}\left(\bar{\ell} \sigma^{I} \gamma^{\mu} i \overleftrightarrow{D}^{\nu} \ell\right) W_{\mu \nu}^{I}$ & \multicolumn{2}{|r|}{$\psi^{2} H^{3}+$ h.c. } \\
\hline $\mathcal{O}_{d W}$ & $\left(\bar{q} \sigma^{\mu \nu} d\right) \sigma^{I} H W_{\mu \nu}^{I}$ & $\mathcal{O}_{\widetilde{W} \ell}^{\prime}$ & $\frac{1}{2}\left(\bar{\ell} \sigma^{I} \gamma^{\mu} i \overleftrightarrow{D}^{\nu} \ell\right) \widetilde{W}_{\mu \nu}^{I}$ & $\mathcal{O}_{u H}$ & $\left(H^{\dagger} H\right) \bar{q} \widetilde{H} u$ \\
\hline $\mathcal{O}_{d B}$ & $\left(\bar{q} \sigma^{\mu \nu} d\right) H B_{\mu \nu}$ & $\mathcal{O}_{B \ell}$ & $\left(\bar{\ell} \gamma^{\mu} \ell\right) \partial^{\nu} B_{\mu \nu}$ & $\mathcal{O}_{d H}$ & $\left(H^{\dagger} H\right) \bar{q} H d$ \\
\hline $\mathcal{O}_{e W}$ & $\left(\bar{\ell} \sigma^{\mu \nu} e\right) \sigma^{I} H W_{\mu \nu}^{I}$ & $\mathcal{O}_{B \ell}^{\prime}$ & $\frac{1}{2}\left(\bar{\ell} \gamma^{\mu} i \overleftrightarrow{D^{\nu}} \ell\right) B_{\mu \nu}$ & $\mathcal{O}_{e H}$ & $\left(H^{\dagger} H\right) \bar{\ell} H e$ \\
\hline $\mathcal{O}_{e B}$ & $\left(\bar{\ell} \sigma^{\mu \nu} e\right) H B_{\mu \nu}$ & $\mathcal{O}_{\widetilde{B} \ell}^{\prime}$ & $\frac{1}{2}\left(\bar{\ell}^{\mu}{ }^{\mu} \overleftrightarrow{D}^{\nu} \ell\right) \widetilde{B}_{\mu \nu}$ & & \\
\hline & & $\begin{array}{l}\mathcal{O}_{B e} \\
\mathcal{O}_{B e}^{\prime} \\
\mathcal{O}_{\widetilde{B} e}^{\prime}\end{array}$ & $\begin{array}{c}\left(\bar{e} \gamma^{\mu} e\right) \partial^{\nu} B_{\mu \nu} \\
\frac{1}{2}\left(\bar{e} \gamma^{\mu} i \overleftrightarrow{D}^{\nu} e\right) B_{\mu \nu} \\
\frac{1}{2}\left(\bar{e} \gamma^{\mu} i \overleftrightarrow{D}^{\nu} e\right) \widetilde{B}_{\mu \nu}\end{array}$ & & \\
\hline
\end{tabular}

Table 8. Two-fermion operators in the Green's basis. Generation indices are suppressed. 


\begin{tabular}{|c|c|c|c|c|c|}
\hline \multicolumn{2}{|c|}{ Four-quark } & \multicolumn{2}{|c|}{ Four-lepton } & \multicolumn{2}{|c|}{ Semileptonic } \\
\hline $\mathcal{O}_{q q}^{(1)}$ & $\left(\bar{q} \gamma^{\mu} q\right)\left(\bar{q} \gamma_{\mu} q\right)$ & $\mathcal{O}_{\ell \ell}$ & $\left(\bar{\ell} \gamma^{\mu} \ell\right)\left(\bar{\ell} \gamma_{\mu} \ell\right)$ & $\mathcal{O}_{\ell q}^{(1)}$ & $\left(\bar{\ell} \gamma^{\mu} \ell\right)\left(\bar{q} \gamma_{\mu} q\right)$ \\
$\mathcal{O}_{q q}^{(3)}$ & $\left(\bar{q} \gamma^{\mu} \sigma^{I} q\right)\left(\bar{q} \gamma_{\mu} \sigma^{I} q\right)$ & $\mathcal{O}_{e e}$ & $\left(\bar{e} \gamma^{\mu} e\right)\left(\bar{e} \gamma_{\mu} e\right)$ & $\mathcal{O}_{\ell q}^{(3)}$ & $\left(\bar{\ell} \gamma^{\mu} \sigma^{I} \ell\right)\left(\bar{q} \gamma_{\mu} \sigma^{I} q\right)$ \\
$\mathcal{O}_{u u}$ & $\left(\bar{u} \gamma^{\mu} u\right)\left(\bar{u} \gamma_{\mu} u\right)$ & $\mathcal{O}_{\ell e}$ & $\left(\bar{\ell} \gamma^{\mu} \ell\right)\left(\bar{e} \gamma_{\mu} e\right)$ & $\mathcal{O}_{e u}$ & $\left(\bar{e} \gamma^{\mu} e\right)\left(\bar{u} \gamma_{\mu} u\right)$ \\
$\mathcal{O}_{d d}$ & $\left(\bar{d} \gamma^{\mu} d\right)\left(\bar{d} \gamma_{\mu} d\right)$ & & & $\mathcal{O}_{e d}$ & $\left(\bar{e} \gamma^{\mu} e\right)\left(\bar{d} \gamma_{\mu} d\right)$ \\
$\mathcal{O}_{u d}^{(1)}$ & $\left(\bar{u} \gamma^{\mu} u\right)\left(\bar{d} \gamma_{\mu} d\right)$ & & & $\mathcal{O}_{q e}$ & $\left(\bar{q} \gamma^{\mu} q\right)\left(\bar{e} \gamma_{\mu} e\right)$ \\
$\mathcal{O}_{u d}^{(8)}$ & $\left(\bar{u} \gamma^{\mu} T^{A} u\right)\left(\bar{d} \gamma_{\mu} T^{A} d\right)$ & & & $\mathcal{O}_{\ell u}$ & $\left(\bar{\ell} \gamma^{\mu} \ell\right)\left(\bar{u} \gamma_{\mu} u\right)$ \\
$\mathcal{O}_{q u}^{(1)}$ & $\left(\bar{q} \gamma^{\mu} q\right)\left(\bar{u} \gamma_{\mu} u\right)$ & & & $\mathcal{O}_{\ell d}$ & $\left(\bar{\ell} \gamma^{\mu} \ell\right)\left(\bar{d} \gamma_{\mu} d\right)$ \\
$\mathcal{O}_{q u}^{(8)}$ & $\left(\bar{q} \gamma^{\mu} T^{A} q\right)\left(\bar{u} \gamma_{\mu} T^{A} u\right)$ & & & $\mathcal{O}_{\ell e d q}$ & $(\bar{\ell} e)(\bar{d} q)$ \\
$\mathcal{O}_{q d}^{(1)}$ & $\left(\bar{q} \gamma^{\mu} q\right)\left(\bar{d} \gamma_{\mu} d\right)$ & & & $\mathcal{O}_{\ell e q u}^{(1)}$ & $\left(\bar{\ell}^{\alpha} e\right) \epsilon_{\alpha \beta}\left(\bar{q}^{\beta} u\right)$ \\
$\mathcal{O}_{q d}^{(8)}$ & $\left(\bar{q} \gamma^{\mu} T^{A} q\right)\left(\bar{d} \gamma_{\mu} T^{A} d\right)$ & & & $\mathcal{O}_{\ell e q u}^{(3)}$ & $\left(\bar{\ell}^{\alpha} \sigma^{\mu \nu} e\right) \epsilon_{\alpha \beta}\left(\bar{q}^{\beta} \sigma_{\mu \nu} u\right)$ \\
$\mathcal{O}_{q u q d}^{(1)}$ & $\left(\bar{q}^{\alpha} u\right) \epsilon_{\alpha \beta}\left(\bar{q}^{\beta} d\right)$ & & & & \\
$\mathcal{O}_{q u q d}^{(8)}$ & $\left(\bar{q}^{\alpha} T^{A} u\right) \epsilon_{\alpha \beta}\left(\bar{q}^{\beta} T^{A} d\right)$ & & & & \\
\hline
\end{tabular}

Table 9. Four-fermion operators. Generation indices are suppressed.

\begin{tabular}{|c|c|}
\hline \multicolumn{2}{|c|}{$B$ and $L$ violating } \\
\hline $\mathcal{O}_{d u q}$ & $\varepsilon_{i j k} \epsilon_{\alpha \beta}\left[\left(d^{i}\right)^{T} C u^{j}\right]\left[\left(q^{k \alpha}\right)^{T} C \ell^{\beta}\right]$ \\
$\mathcal{O}_{q q u}$ & $\varepsilon_{i j k} \epsilon_{\alpha \beta}\left[\left(q^{i \alpha}\right)^{T} C q^{j \beta}\right]\left[\left(u^{k}\right)^{T} C e\right]$ \\
$\mathcal{O}_{q q q}$ & $\varepsilon_{i j k} \epsilon_{\alpha \beta} \epsilon_{\gamma \delta}\left[\left(q^{i \alpha}\right)^{T} C q^{j \gamma}\right]\left[\left(q^{k \delta}\right)^{T} C \ell^{\beta}\right]$ \\
$\mathcal{O}_{d u u}$ & $\varepsilon_{i j k}\left[\left(d^{i}\right)^{T} C u^{j}\right]\left[\left(u^{k}\right)^{T} C e\right]$ \\
\hline
\end{tabular}

Table 10. Baryon and lepton number violating four-fermion operators. Generation indices are suppressed and $C$ is the Dirac charge conjugation matrix. 


\section{Auxiliary expressions from the UOLEA}

We append here expressions for the UOLEA coefficients that arise in case of possible LQs mass degeneracies. We split them as $f_{n}=\frac{i}{16 \pi^{2}} \tilde{f}_{n}$ listing only $\tilde{f}_{n}$. We also adopt the notation $\Delta_{i j}^{2}=M_{i}^{2}-M_{j}^{2}$ and wherever $S_{i}=\left\{S_{1}, \tilde{S}_{2}\right\}$. The coefficients then read,

$$
\begin{aligned}
& \tilde{f}_{11}^{S_{i} S_{j} S_{i}}=\frac{2 M_{i}^{6}+M_{j}^{6}+3 M_{i}^{2} M_{j}^{2}\left(M_{i}^{2}-2 M_{j}^{2}\right)+M_{i}^{4} M_{j}^{2} \log M_{j}^{2} / M_{i}^{2}}{6 M_{i}^{2}\left(\Delta_{i j}\right)^{4}}, \\
& \tilde{f}_{11}^{S_{i} S_{i} S_{j}}=\frac{M_{i}^{4}+4 M_{i}^{2} M_{j}^{2}\left(1+\log M_{j}^{2} / M_{i}^{2}\right)+2 M_{j}^{4}\left(\log M_{j}^{2} / M_{i}^{2}-5 / 2\right)}{2\left(\Delta_{i j}\right)^{4}}, \\
& \tilde{f}_{12}^{S_{i} S_{j}}=\frac{M_{i}^{4}+10 M_{i}^{2} M_{j}^{2}+M_{j}^{4}}{12\left(\Delta_{i j}^{2}\right)^{4}}-\frac{M_{i}^{2} M_{j}^{2}\left(M_{i}^{2}+M_{j}^{2}\right) \log M_{i}^{2} / M_{j}^{2}}{2\left(\Delta_{i j}^{2}\right)^{5}}, \\
& \tilde{f}_{13}^{S_{i} S_{j}}=\frac{2 M_{i}^{4}+5 M_{i}^{2} M_{j}^{2}-M_{j}^{4}}{12 M_{i}^{2}\left(\Delta_{i j}^{2}\right)^{3}}-\frac{M_{i}^{2} M_{j}^{2} \log M_{i}^{2} / M_{j}^{2}}{\left(\Delta_{i j}^{2}\right)^{5}} \\
& \tilde{f}_{14}^{S_{i} S_{j}}=-\frac{M_{i}^{4}+10 M_{i}^{2} M_{j}^{2} M_{j}^{4}}{6\left(\Delta_{i j}^{2}\right)^{4}}+\frac{M_{i}^{2} M_{j}^{2}\left(M_{i}^{2} M_{j}^{2}\right) \log M_{i}^{2} / M_{j}^{2}}{\left(\Delta_{i j}^{2}\right)^{5}}, \\
& \tilde{f}_{17}^{S_{i} S_{j} S_{i} S_{j}}=\tilde{f}_{17}^{S_{i} S_{j}} \\
& =\frac{18 M_{j}^{2}\left(M_{i}^{4}+M_{i}^{2} M_{j}^{2}\right)-2 M_{i}^{6}-34 M_{j}^{6}+12\left(M_{j}^{4}+3 M_{i}^{2} M_{j}^{2}\right) \log M_{j}^{2} / M_{i}^{2}}{12 M_{j}^{2}\left(\Delta_{i j}^{2}\right)^{5}}, \\
& \tilde{f}_{18}^{S_{i} S_{j} S_{i} S_{j}}=\tilde{f}_{18}^{S_{i} S_{j}}=\frac{M_{i}^{2}+M_{j}^{2}}{6\left(\Delta_{i j}^{2}\right)^{4}}+\frac{M_{j}^{8}-M_{i}^{8}}{12 M_{i}^{2} M_{j}^{2}\left(\Delta_{i j}^{2}\right)^{5}}+\frac{M_{i}^{2} M_{j}^{2} \log M_{j}^{2} / M_{i}^{2}}{\left(\Delta_{i j}^{2}\right)^{5}}, \\
& \tilde{f}_{19}^{S_{i} S_{j} S_{i} S_{j} S_{i} S_{j}}=\tilde{f}_{19}^{S_{i} S_{j}}=\frac{\left(M_{i}^{6}-M_{j}^{6}\right)+9 M_{i}^{2} M_{j}^{2}\left(\Delta_{i j}^{2}\right)+6 M_{i}^{2} M_{j}^{2}\left(M_{i}^{2}+M_{j}^{2}\right) \log M_{j}^{2} / M_{i}^{2}}{12 M_{i}^{2} M_{j}^{2}\left(\Delta_{i j}^{2}\right)^{5}}
\end{aligned}
$$

Open Access. This article is distributed under the terms of the Creative Commons Attribution License (CC-BY 4.0), which permits any use, distribution and reproduction in any medium, provided the original author(s) and source are credited.

\section{References}

[1] S. Weinberg, Effective gauge theories, Phys. Lett. B 91 (1980) 51 [INSPIRE].

[2] C.G. Callan, Jr., S.R. Coleman, J. Wess and B. Zumino, Structure of phenomenological lagrangians. 2, Phys. Rev. 177 (1969) 2247 [INSPIRE].

[3] S.R. Coleman, J. Wess and B. Zumino, Structure of phenomenological Lagrangians. 1, Phys. Rev. 177 (1969) 2239 [INSPIRE].

[4] T. Appelquist and J. Carazzone, Infrared singularities and massive fields, Phys. Rev. D 11 (1975) 2856 [INSPIRE].

[5] M.K. Gaillard, The effective one loop Lagrangian with derivative couplings, Nucl. Phys. B 268 (1986) 669 [INSPIRE].

[6] O. Cheyette, Effective action for the standard model with large Higgs mass, Nucl. Phys. B 297 (1988) 183 [INSPIRE]. 
[7] L.-H. Chan, Derivative expansion for the one loop effective actions with internal symmetry, Phys. Rev. Lett. 57 (1986) 1199 [INSPIRE].

[8] B. Henning, X. Lu and H. Murayama, How to use the Standard Model effective field theory, JHEP 01 (2016) 023 [arXiv:1412.1837] [INSPIRE].

[9] B. Henning, X. Lu and H. Murayama, One-loop matching and running with covariant derivative expansion, JHEP 01 (2018) 123 [arXiv:1604.01019] [INSPIRE].

[10] A. Drozd, J. Ellis, J. Quevillon and T. You, The Universal one-loop effective action, JHEP 03 (2016) 180 [arXiv: 1512.03003] [INSPIRE].

[11] S.A.R. Ellis, J. Quevillon, T. You and Z. Zhang, Mixed heavy-light matching in the universal one-loop effective action, Phys. Lett. B $\mathbf{7 6 2}$ (2016) 166 [arXiv:1604.02445] [INSPIRE].

[12] S.A.R. Ellis, J. Quevillon, T. You and Z. Zhang, Extending the universal one-loop effective action: heavy-light coefficients, JHEP 08 (2017) 054 [arXiv:1706.07765] [INSPIRE].

[13] A. Angelescu and P. Huang, Integrating out new fermions at one loop, JHEP 01 (2021) 049 [arXiv: 2006.16532] [INSPIRE].

[14] S.A.R. Ellis, J. Quevillon, P.N.H. Vuong, T. You and Z. Zhang, The fermionic universal one-loop effective action, JHEP 11 (2020) 078 [arXiv: 2006.16260] [INSPIRE].

[15] M. Krämer, B. Summ and A. Voigt, Completing the scalar and fermionic universal one-loop effective action, JHEP 01 (2020) 079 [arXiv:1908.04798] [INSPIRE].

[16] Z. Zhang, Covariant diagrams for one-loop matching, JHEP 05 (2017) 152 [arXiv: 1610.00710] [INSPIRE].

[17] M. Beneke and V.A. Smirnov, Asymptotic expansion of Feynman integrals near threshold, Nucl. Phys. B 522 (1998) 321 [hep-ph/9711391] [INSPIRE].

[18] B. Jantzen, Foundation and generalization of the expansion by regions, JHEP 12 (2011) 076 [arXiv:1111.2589] [INSPIRE].

[19] J. Fuentes-Martin, J. Portoles and P. Ruiz-Femenia, Integrating out heavy particles with functional methods: a simplified framework, JHEP 09 (2016) 156 [arXiv:1607.02142] [INSPIRE].

[20] S. Dittmaier and C. Grosse-Knetter, Deriving nondecoupling effects of heavy fields from the path integral: A Heavy Higgs field in an SU(2) gauge theory, Phys. Rev. D 52 (1995) 7276 [hep-ph/9501285] [INSPIRE].

[21] S. Dittmaier and C. Grosse-Knetter, Integrating out the standard Higgs field in the path integral, Nucl. Phys. B 459 (1996) 497 [hep-ph/9505266] [INSPIRE].

[22] J.D. Wells and Z. Zhang, Effective field theory approach to trans-TeV supersymmetry: covariant matching, Yukawa unification and Higgs couplings, JHEP 05 (2018) 182 [arXiv: 1711.04774] [INSPIRE].

[23] T. Cohen, X. Lu and Z. Zhang, Functional prescription for EFT matching, JHEP 02 (2021) 228 [arXiv: 2011.02484] [INSPIRE].

[24] T. Cohen, X. Lu and Z. Zhang, STrEAMlining EFT matching, SciPost Phys. 10 (2021) 098 [arXiv: 2012.07851] [INSPIRE].

[25] J. Fuentes-Martin, M. König, J. Pagès, A.E. Thomsen and F. Wilsch, SuperTracer: a calculator of functional supertraces for one-loop EFT matching, JHEP 04 (2021) 281 [arXiv: 2012.08506] [INSPIRE]. 
[26] K. Finn, S. Karamitsos and A. Pilaftsis, Frame covariant formalism for fermionic theories, Eur. Phys. J. C 81 (2021) 572 [arXiv: 2006.05831] [InSPIRE].

[27] W. Buchmüller, R. Ruckl and D. Wyler, Leptoquarks in lepton-quark collisions, Phys. Lett. B 191 (1987) 442 [Erratum ibid. 448 (1999) 320] [INSPIRE].

[28] D. Zhang and S. Zhou, Complete one-loop matching of the type-I seesaw model onto the standard model effective field theory, arXiv:2107.12133 [INSPIRE].

[29] I. Brivio et al., From models to SMEFT and back?, arXiv:2108.01094 [INSPIRE].

[30] S. Das Bakshi, J. Chakrabortty and M. Spannowsky, Classifying standard model extensions effectively with precision observables, Phys. Rev. D 103 (2021) 056019 [arXiv:2012.03839] [INSPIRE].

[31] S. Das Bakshi, J. Chakrabortty and S.K. Patra, CoDEx: Wilson coefficient calculator connecting SMEFT to UV theory, Eur. Phys. J. C 79 (2019) 21 [arXiv:1808.04403] [INSPIRE].

[32] M. Jiang, N. Craig, Y.-Y. Li and D. Sutherland, Complete one-loop matching for a singlet scalar in the Standard Model EFT, JHEP 02 (2019) 031 [Erratum ibid. 01 (2021) 135] [arXiv: 1811.08878] [INSPIRE].

[33] V. Gherardi, D. Marzocca and E. Venturini, Matching scalar leptoquarks to the SMEFT at one loop, JHEP 07 (2020) 225 [Erratum ibid. 01 (2021) 006] [arXiv:2003.12525] [INSPIRE].

[34] B. Grzadkowski, M. Iskrzynski, M. Misiak and J. Rosiek, Dimension-six terms in the standard model Lagrangian, JHEP 10 (2010) 085 [arXiv: 1008.4884] [INSPIRE].

[35] M.S. Bilenky and A. Santamaria, One loop effective Lagrangian for a standard model with a heavy charged scalar singlet, Nucl. Phys. B 420 (1994) 47 [hep-ph/9310302] [INSPIRE].

[36] K. Mantzaropoulos, Effective field theories and one-loop matching, M.Sc. thesis, University of Ioannina, Greece (2019).

[37] J. de Blas, J.C. Criado, M. Pérez-Victoria and J. Santiago, Effective description of general extensions of the Standard Model: the complete tree-level dictionary, JHEP 03 (2018) 109 [arXiv: 1711.10391] [INSPIRE].

[38] I. Doršner, S. Fajfer, A. Greljo, J.F. Kamenik and N. Košnik, Physics of leptoquarks in precision experiments and at particle colliders, Phys. Rept. 641 (2016) 1 [arXiv: 1603.04993] [INSPIRE].

[39] M. Bauer and M. Neubert, Minimal leptoquark explanation for the $R_{D^{(*)}}, R_{K}$, and $(g-2)_{\mu}$ Anomalies, Phys. Rev. Lett. 116 (2016) 141802 [arXiv:1511.01900] [INSPIRE].

[40] A. Angelescu, D. Bečirević, D.A. Faroughy, F. Jaffredo and O. Sumensari, Single leptoquark solutions to the B-physics anomalies, Phys. Rev. D 104 (2021) 055017 [arXiv:2103.12504] [INSPIRE].

[41] A. Crivellin, C. Greub, D. Müller and F. Saturnino, Scalar leptoquarks in leptonic processes, JHEP 02 (2021) 182 [arXiv: 2010.06593] [INSPIRE].

[42] V. Gherardi, D. Marzocca and E. Venturini, Low-energy phenomenology of scalar leptoquarks at one-loop accuracy, JHEP 01 (2021) 138 [arXiv: 2008.09548] [INSPIRE].

[43] L. Alasfar, A. Azatov, J. de Blas, A. Paul and M. Valli, $B$ anomalies under the lens of electroweak precision, JHEP 12 (2020) 016 [arXiv: 2007.04400] [INSPIRE]. 
[44] M. Bordone, O. Catà, T. Feldmann and R. Mandal, Constraining flavour patterns of scalar leptoquarks in the effective field theory, JHEP 03 (2021) 122 [arXiv:2010.03297] [INSPIRE].

[45] M.J. Dugan and B. Grinstein, On the vanishing of evanescent operators, Phys. Lett. B 256 (1991) 239 [INSPIRE].

[46] S. Herrlich and U. Nierste, Evanescent operators, scheme dependences and double insertions, Nucl. Phys. B 455 (1995) 39 [hep-ph/9412375] [INSPIRE].

[47] W. Dekens and P. Stoffer, Low-energy effective field theory below the electroweak scale: matching at one loop, JHEP 10 (2019) 197 [arXiv: 1908.05295] [INSPIRE].

[48] J. Aebischer, C. Bobeth, A.J. Buras and J. Kumar, SMEFT ATLAS of $\triangle F=2$ transitions, JHEP 12 (2020) 187 [arXiv:2009.07276] [INSPIRE].

[49] I. Dorsner, A scalar leptoquark in SU(5), Phys. Rev. D 86 (2012) 055009 [arXiv:1206.5998] [INSPIRE].

[50] I. Doršner, S. Fajfer and N. Košnik, Leptoquark mechanism of neutrino masses within the grand unification framework, Eur. Phys. J. C 77 (2017) 417 [arXiv:1701.08322] [InSPIRE].

[51] I. Dorsner, S. Fajfer and N. Kosnik, Heavy and light scalar leptoquarks in proton decay, Phys. Rev. D 86 (2012) 015013 [arXiv: 1204.0674] [INSPIRE].

[52] J. Heeck and V. Takhistov, Inclusive nucleon decay searches as a frontier of baryon number violation, Phys. Rev. D 101 (2020) 015005 [arXiv:1910.07647] [INSPIRE].

[53] U. Mahanta, Neutrino masses and mixing angles from leptoquark interactions, Phys. Rev. D 62 (2000) 073009 [hep-ph/9909518] [INSPIRE].

[54] D. Zhang, Radiative neutrino masses, lepton flavor mixing and muon $g-2$ in a leptoquark model, JHEP 07 (2021) 069 [arXiv:2105.08670] [INSPIRE].

[55] A. Crivellin and M. Hoferichter, Consequences of chirally enhanced explanations of $(g-2)_{\mu}$ for $h \rightarrow \mu \mu$ and $Z \rightarrow \mu \mu$, JHEP 07 (2021) 135 [arXiv:2104.03202] [INSPIRE].

[56] P. Athron, C. Balázs, D.H. Jacob, W. Kotlarski, D. Stöckinger and H. Stöckinger-Kim, New physics explanations of $a_{\mu}$ in light of the FNAL muon $g-2$ measurement, JHEP 09 (2021) 080 [arXiv: 2104.03691] [INSPIRE].

[57] A. Dedes, W. Materkowska, M. Paraskevas, J. Rosiek and K. Suxho, Feynman rules for the standard model effective field theory in $R_{\xi}$-gauges, JHEP 06 (2017) 143 [arXiv:1704.03888] [INSPIRE].

[58] J.M. Arnold, B. Fornal and M.B. Wise, Phenomenology of scalar leptoquarks, Phys. Rev. D 88 (2013) 035009 [arXiv: 1304.6119] [InSPIRE].

[59] C. Murgui and M.B. Wise, Scalar leptoquarks, baryon number violation, and Pati-Salam symmetry, Phys. Rev. D 104 (2021) 035017 [arXiv:2105.14029] [InSPIRE].

[60] L. Lehman, Extending the standard model effective field theory with the complete set of dimension-7 operators, Phys. Rev. D 90 (2014) 125023 [arXiv:1410.4193] [INSPIRE].

[61] A. Kobach, Baryon number, lepton number, and operator dimension in the standard model, Phys. Lett. B 758 (2016) 455 [arXiv: 1604.05726] [InSPIRE].

[62] A. Helset and A. Kobach, Baryon number, lepton number, and operator dimension in the SMEFT with flavor symmetries, Phys. Lett. B 800 (2020) 135132 [arXiv: 1909.05853] [INSPIRE]. 
[63] A.J. Buras and P.H. Weisz, Qcd nonleading corrections to weak decays in dimensional regularization and 't hooft-veltman schemes, Nucl. Phys. B 333 (1990) 66.

[64] E.E. Jenkins, A.V. Manohar and M. Trott, Renormalization group evolution of the standard model dimension six operators. Part I. Formalism and lambda dependence, JHEP 10 (2013) 087 [arXiv: 1308.2627] [inSPIRE].

[65] E.E. Jenkins, A.V. Manohar and M. Trott, Renormalization group evolution of the standard model dimension six operators. Part II. Yukawa dependence, JHEP 01 (2014) 035 [arXiv: 1310.4838] [INSPIRE].

[66] R. Alonso, E.E. Jenkins, A.V. Manohar and M. Trott, Renormalization group evolution of the standard model dimension six operators. Part III. Gauge coupling dependence and phenomenology, JHEP 04 (2014) 159 [arXiv:1312.2014] [INSPIRE].

[67] A. Celis, J. Fuentes-Martin, A. Vicente and J. Virto, DsixTools: the standard model effective field theory toolkit, Eur. Phys. J. C 77 (2017) 405 [arXiv: 1704.04504] [InSPIRE].

[68] Muon G-2 collaboration, Measurement of the positive muon anomalous magnetic moment to 0.46 ppm, Phys. Rev. Lett. 126 (2021) 141801 [arXiv:2104.03281] [INSPIRE].

[69] Muon G-2 collaboration, Final report of the Muon E821 anomalous magnetic moment measurement at BNL, Phys. Rev. D 73 (2006) 072003 [hep-ex/0602035] [inSPIRE].

[70] J. Aebischer, W. Dekens, E.E. Jenkins, A.V. Manohar, D. Sengupta and P. Stoffer, Effective field theory interpretation of lepton magnetic and electric dipole moments, JHEP 07 (2021) 107 [arXiv : 2102.08954] [INSPIRE].

[71] ACME collaboration, Improved limit on the electric dipole moment of the electron, Nature 562 (2018) 355 [INSPIRE].

[72] A. Dedes, H.E. Haber and J. Rosiek, Seesaw mechanism in the sneutrino sector and its consequences, JHEP 11 (2007) 059 [arXiv:0707.3718] [INSPIRE].

[73] G. Panico, A. Pomarol and M. Riembau, EFT approach to the electron Electric Dipole Moment at the two-loop level, JHEP 04 (2019) 090 [arXiv:1810.09413] [INSPIRE].

[74] W. Dekens, J. de Vries, M. Jung and K.K. Vos, The phenomenology of electric dipole moments in models of scalar leptoquarks, JHEP 01 (2019) 069 [arXiv:1809.09114] [INSPIRE].

[75] Super-Kamiokande collaboration, Search for proton decay via $p \rightarrow e^{+} \pi^{0}$ and $p \rightarrow \mu^{+} \pi^{0}$ in 0.31 megaton - years exposure of the Super-Kamiokande water Cherenkov detector, Phys. Rev. D 95 (2017) 012004 [arXiv: 1610.03597] [INSPIRE].

[76] Super-Kamiokande collaboration, Search for proton decay via $p \rightarrow \nu K^{+}$using 260 kiloton - year data of Super-Kamiokande, Phys. Rev. D 90 (2014) 072005 [arXiv:1408.1195] [INSPIRE].

[77] P. Nath and P. Fileviez Perez, Proton stability in grand unified theories, in strings and in branes, Phys. Rept. 441 (2007) 191 [hep-ph/0601023] [INSPIRE].

[78] I. Baldes, N.F. Bell and R.R. Volkas, Baryon number violating scalar diquarks at the LHC, Phys. Rev. D 84 (2011) 115019 [arXiv:1110.4450] [INSPIRE].

[79] T. Hambye and J. Heeck, Proton decay into charged leptons, Phys. Rev. Lett. 120 (2018) 171801 [arXiv: 1712.04871] [INSPIRE]. 
[80] J.C. Helo, M. Hirsch and T. Ota, Proton decay at one loop, Phys. Rev. D 99 (2019) 095021 [arXiv: 1904.00036] [INSPIRE].

[81] R. Coy and M. Frigerio, Effective comparison of neutrino-mass models, arXiv:2110.09126 [INSPIRE].

[82] A. Crivellin and L. Schnell, Complete Lagrangian and set of Feynman rules for scalar leptoquarks, Comput. Phys. Commun. 271 (2022) 108188 [arXiv:2105.04844] [INSPIRE]. 